\title{
Preparation of 1-Azido-2-Bromo-1,1,2,2-Tetrafluoroethane and Its Use in the Synthesis of N-Fluoroalkylated Nitrogen Heterocycles
}

David Tichý, ${ }^{\dagger,}{ }^{\dagger}$ Vojtěch Košt’ál, ${ }^{\dagger}$ Vladimir Motornov, ${ }^{\dagger, \S}$ Iveta Klimánková, ${ }^{\dagger}$ and Petr Beier ${ }^{*}, \dagger$

†nstitute of Organic Chemistry and Biochemistry of the Czech Academy of Sciences, Flemingovo nam., 2, 166 10, Prague 6, Czech Republic. E-mail: beier@uochb.cas.cz

${ }^{\ddagger}$ Department of Organic Chemistry, Faculty of Science, Charles University, Hlavova 2030/8, 12843 Prague, Czech Republic.

${ }^{\S}$ Higher Chemical College, D. I. Mendeleev University of the Chemical Technology of Russia, Miusskaya sq. 9, 125047, Moscow, Russian Federation.

Table of Contents

Copies of NMR spectra

S2-S47 


\section{Copies of NMR spectra}

${ }^{13} \mathrm{C}\left\{{ }^{1} \mathrm{H}\right\}$ NMR spectrum of $1\left(\mathrm{CDCl}_{3}, 101 \mathrm{MHz}\right)$

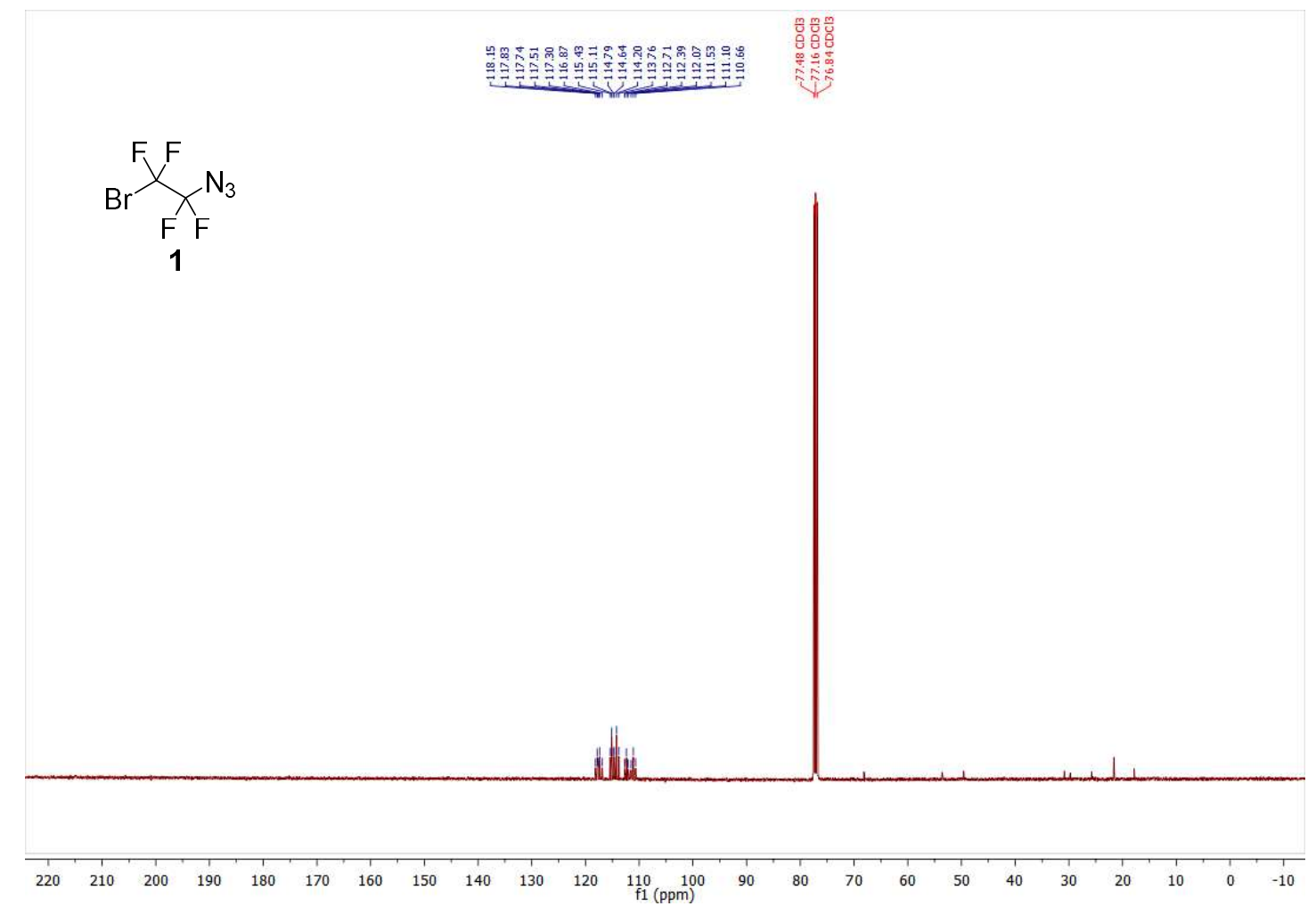

${ }^{19} \mathrm{~F}$ NMR spectrum of $1\left(\mathrm{CDCl}_{3}, 377 \mathrm{MHz}\right)$

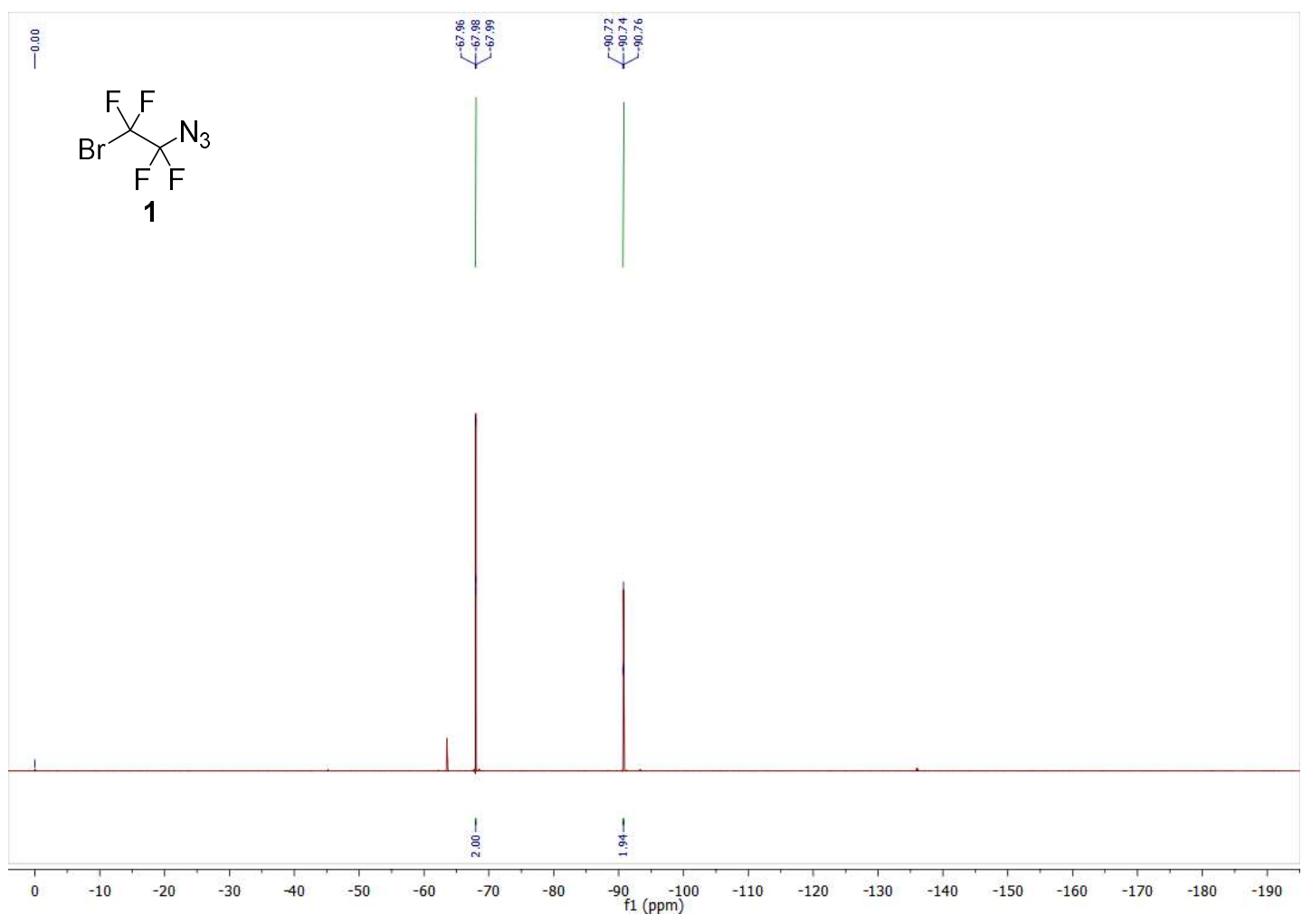


${ }^{1} \mathrm{H}$ NMR spectrum of $2 \mathrm{a}\left(\mathrm{CDCl}_{3}, 400 \mathrm{MHz}\right)$

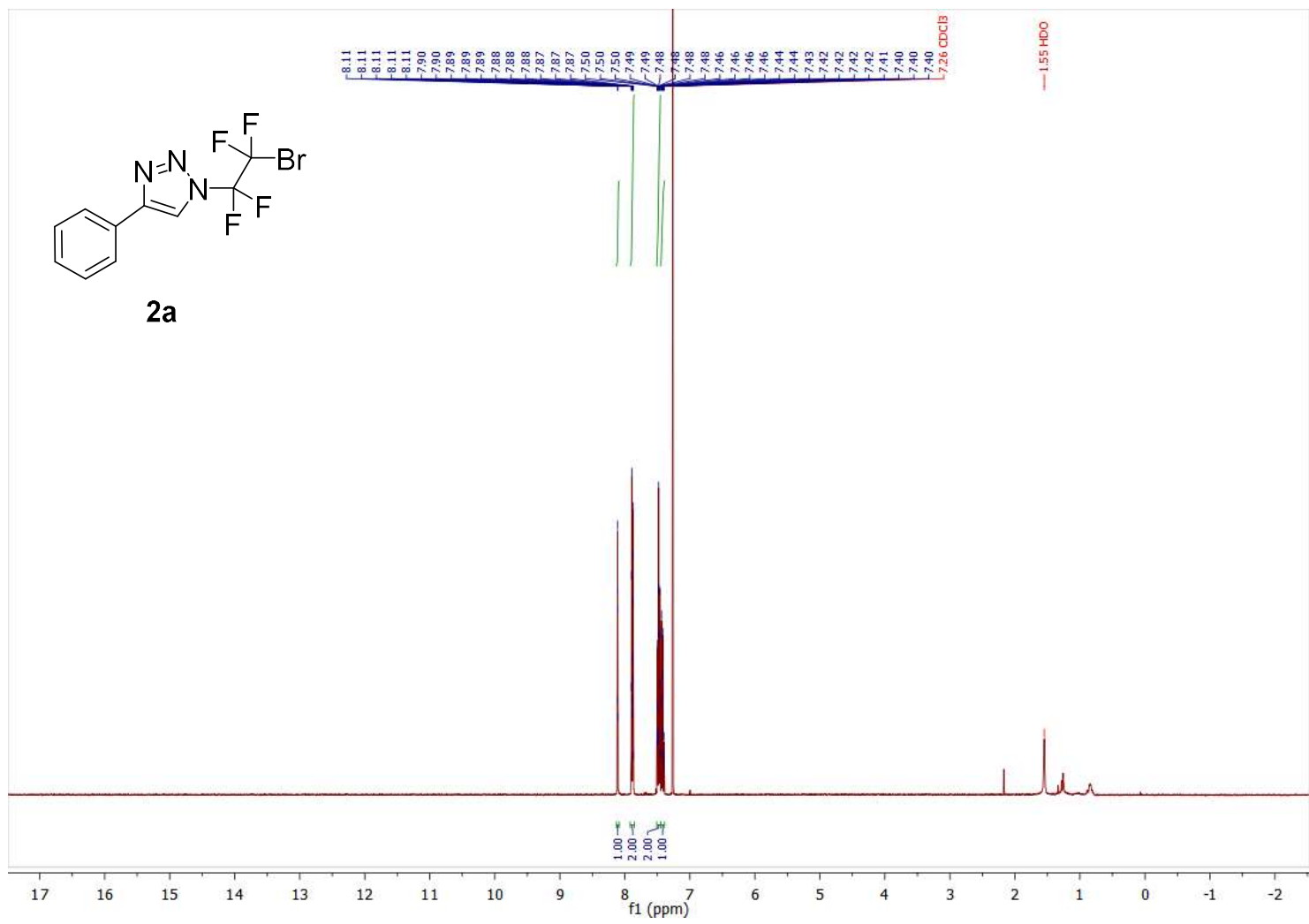

${ }^{13} \mathrm{C}\left\{{ }^{1} \mathrm{H}\right\}$ NMR spectrum of $\mathbf{2 a}\left(\mathrm{CDCl}_{3}, 101 \mathrm{MHz}\right)$

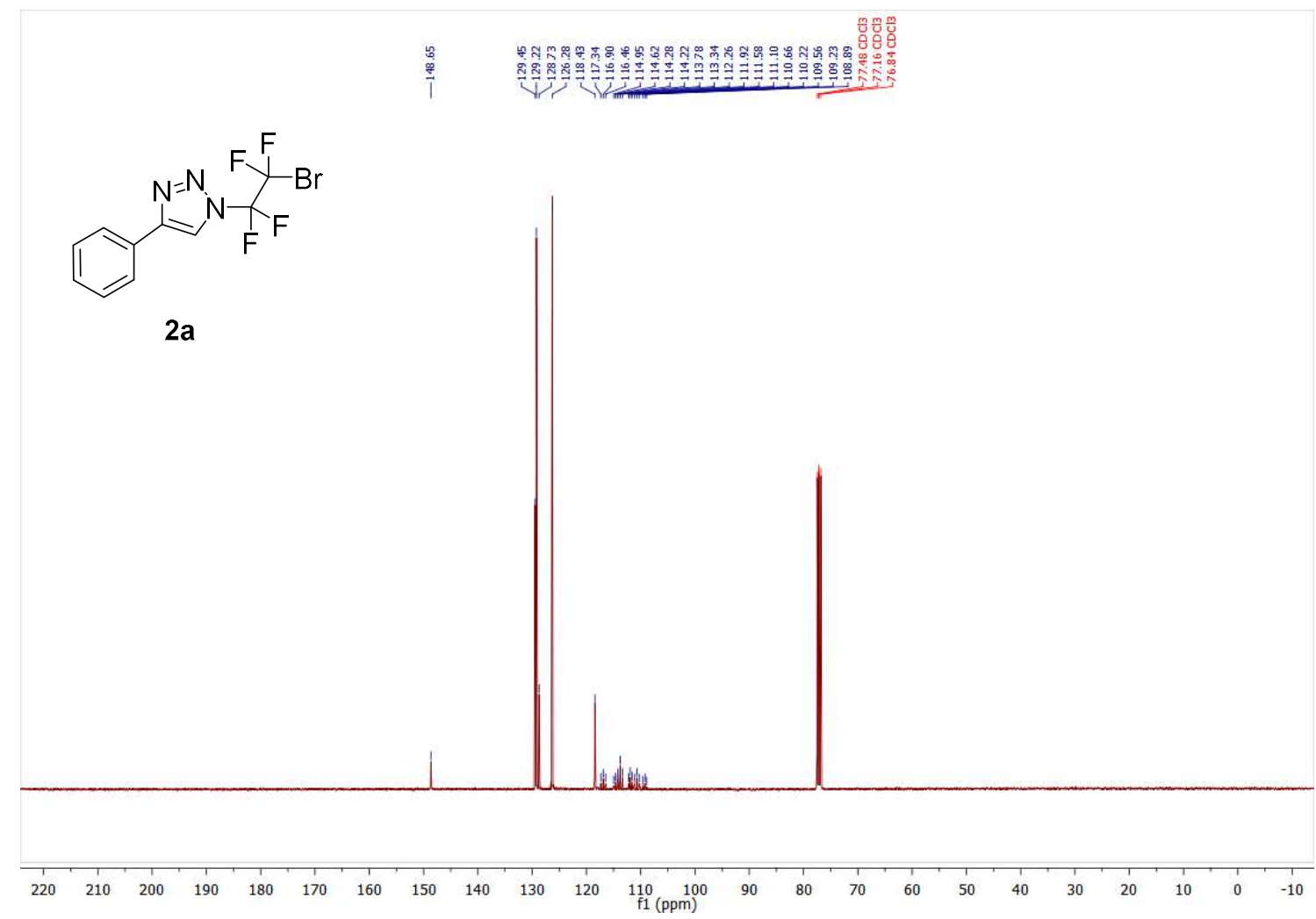


${ }^{19} \mathrm{~F}$ NMR spectrum of $\mathbf{2 a}\left(\mathrm{CDCl}_{3}, 377 \mathrm{MHz}\right)$$$
\text { 辛 }
$$
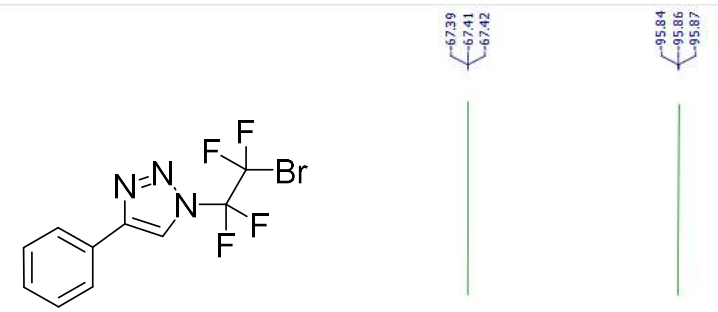

$2 a$

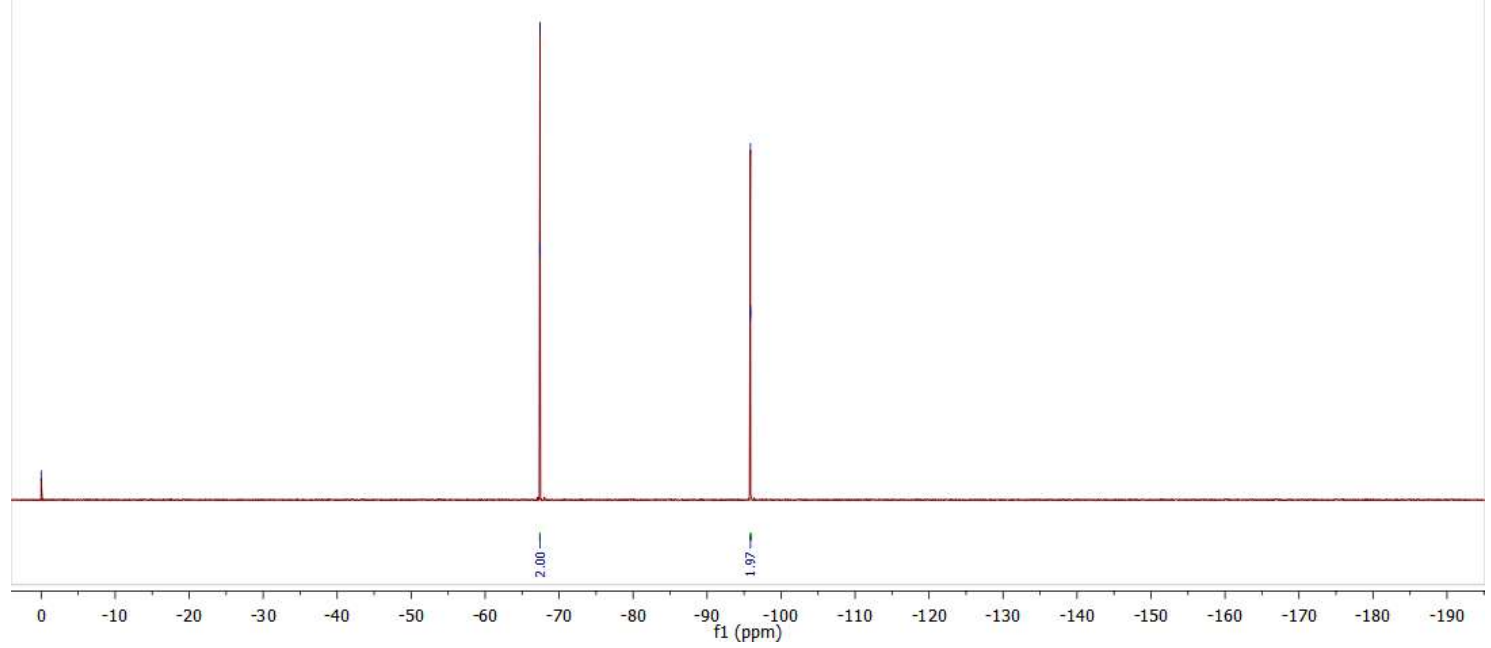

${ }^{1} \mathrm{H}$ NMR spectrum of $\mathbf{2} \mathbf{b}\left(\mathrm{CDCl}_{3}, 400 \mathrm{MHz}\right)$

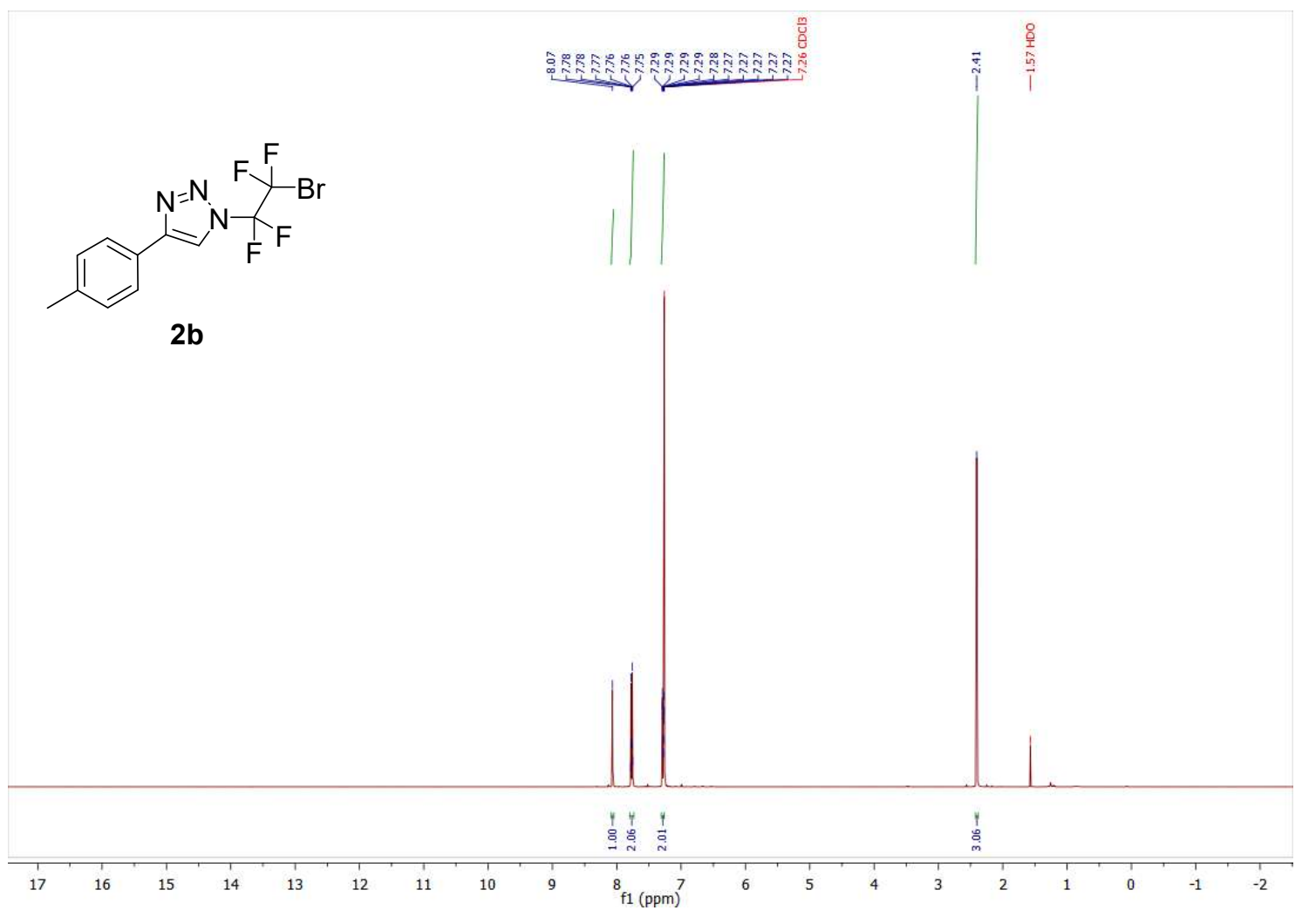


${ }^{13} \mathrm{C}\left\{{ }^{1} \mathrm{H}\right\}$ NMR spectrum of $\mathbf{2} \mathbf{b}\left(\mathrm{CDCl}_{3}, 101 \mathrm{MHz}\right)$

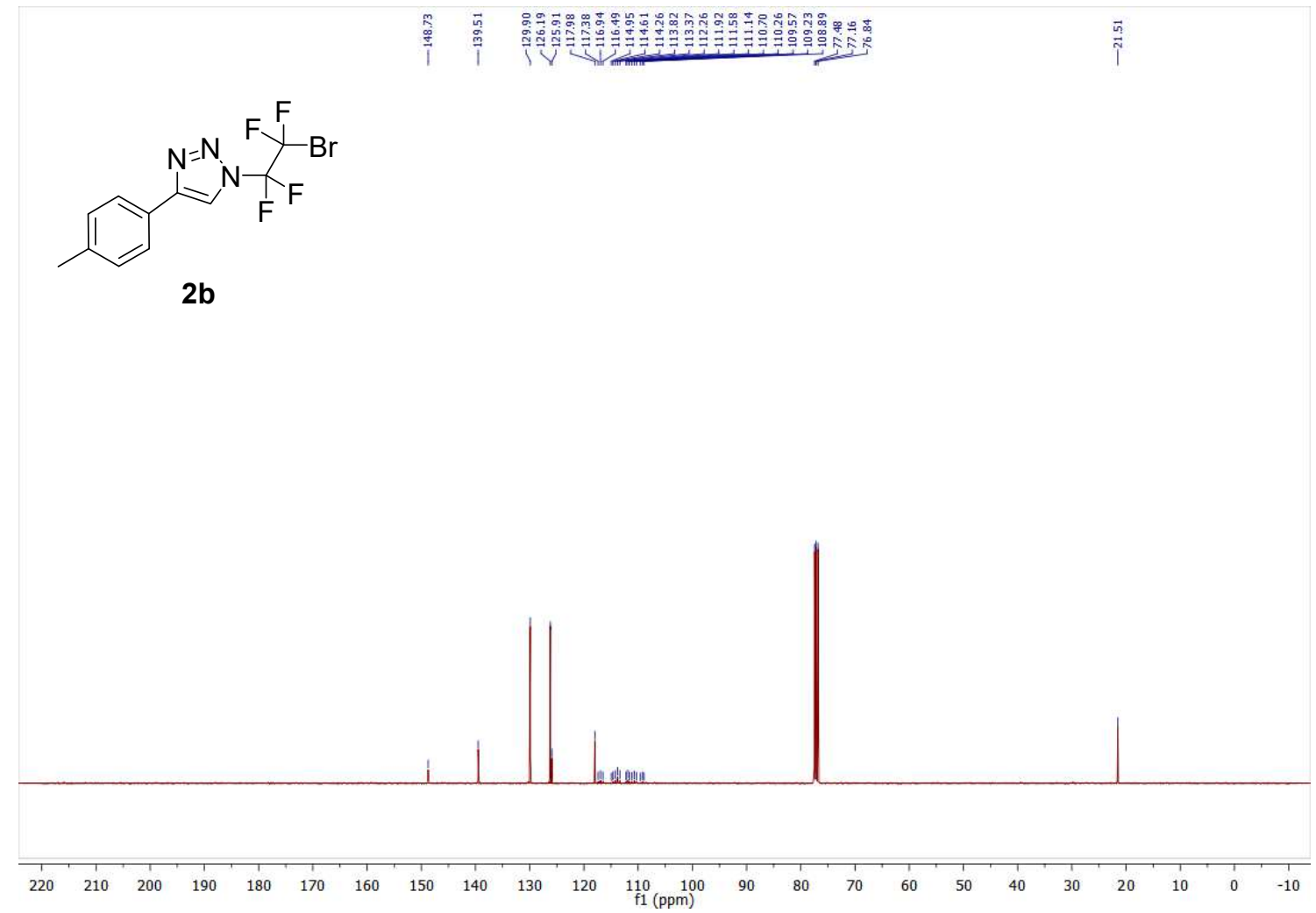

${ }^{19} \mathrm{~F}$ NMR spectrum of $\mathbf{2} \mathbf{b}\left(\mathrm{CDCl}_{3}, 377 \mathrm{MHz}\right)$

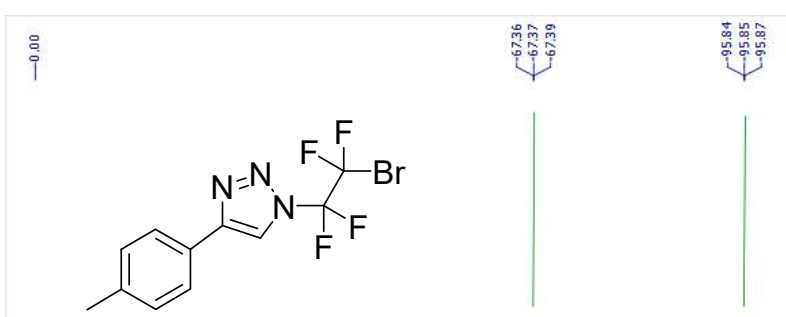

2b

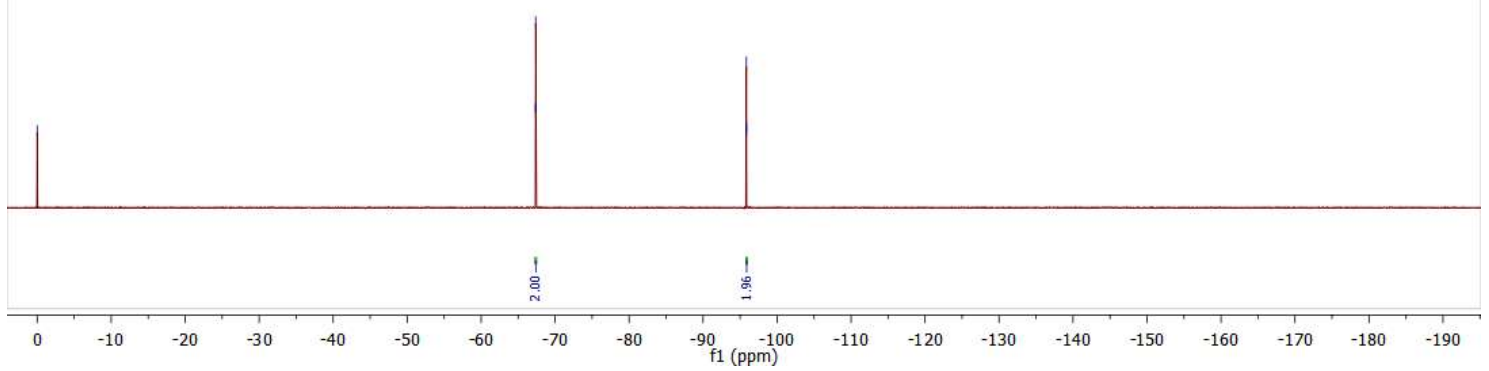


${ }^{1} \mathrm{H}$ NMR spectrum of $\mathbf{2 c}\left(\mathrm{CDCl}_{3}, 400 \mathrm{MHz}\right)$

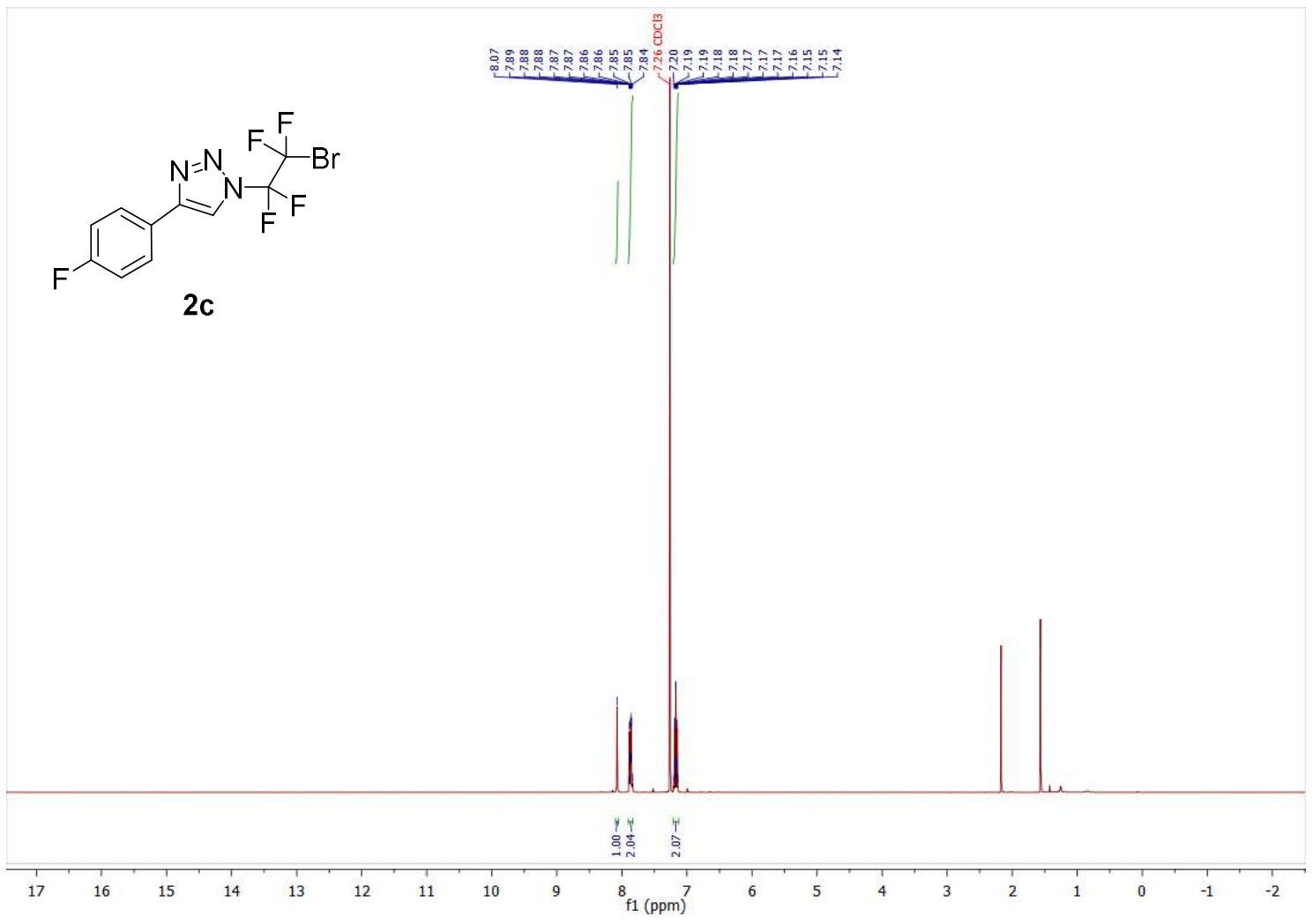

${ }^{13} \mathrm{C}\left\{{ }^{1} \mathrm{H}\right\}$ NMR spectrum of $2 \mathrm{c}\left(\mathrm{CDCl}_{3}, 101 \mathrm{MHz}\right)$

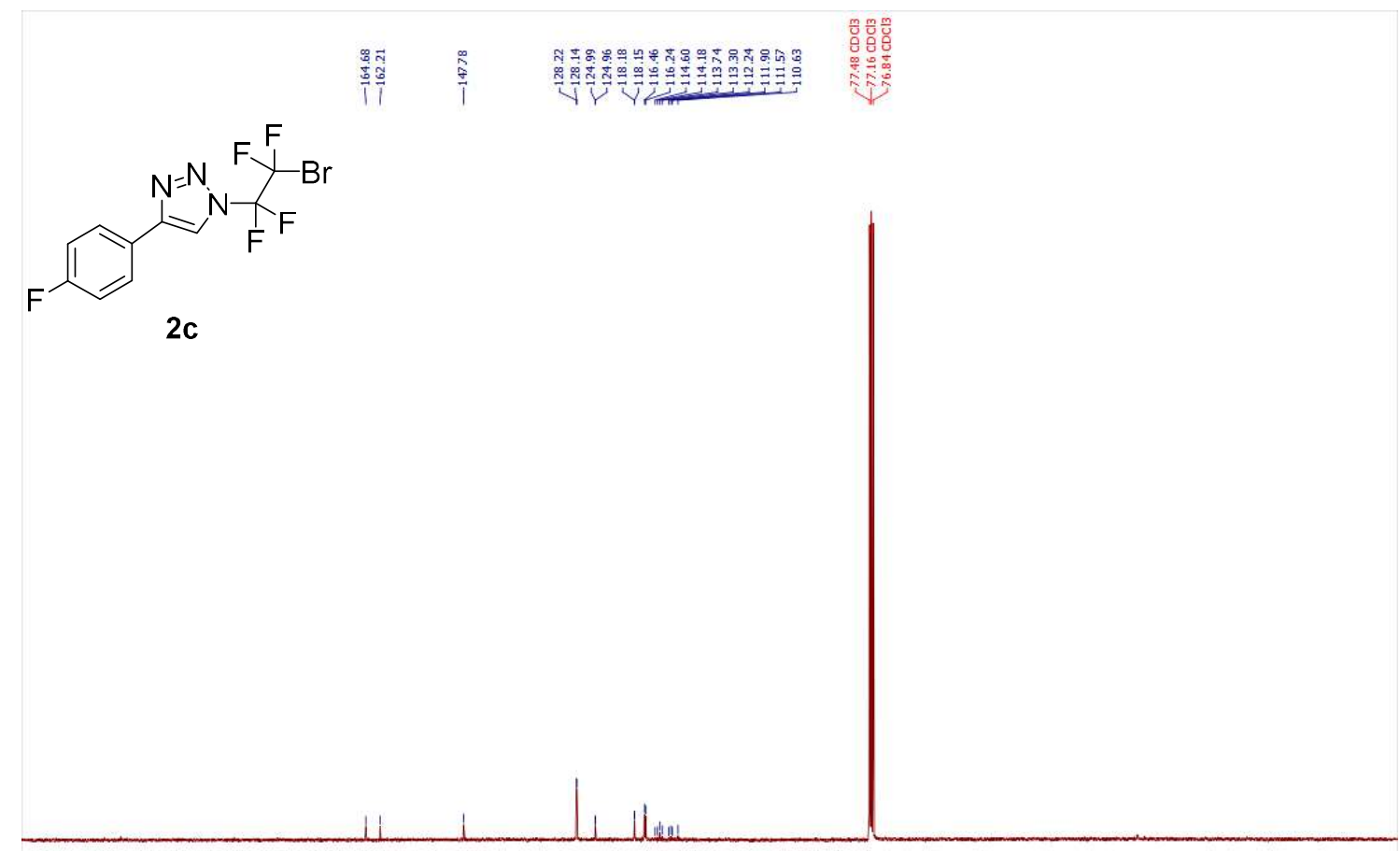

$\begin{array}{lllllllllllllllllllllllll}220 & 210 & 200 & 190 & 180 & 170 & 160 & 150 & 140 & 130 & 120 & \underset{\mathrm{f} 1(\mathrm{ppm})}{110} & 90 & 80 & 70 & 60 & 50 & 40 & 30 & 20 & 10 & 0 & -10\end{array}$ 
${ }^{19} \mathrm{~F}$ NMR spectrum of $2 \mathrm{c}\left(\mathrm{CDCl}_{3}, 377 \mathrm{MHz}\right)$

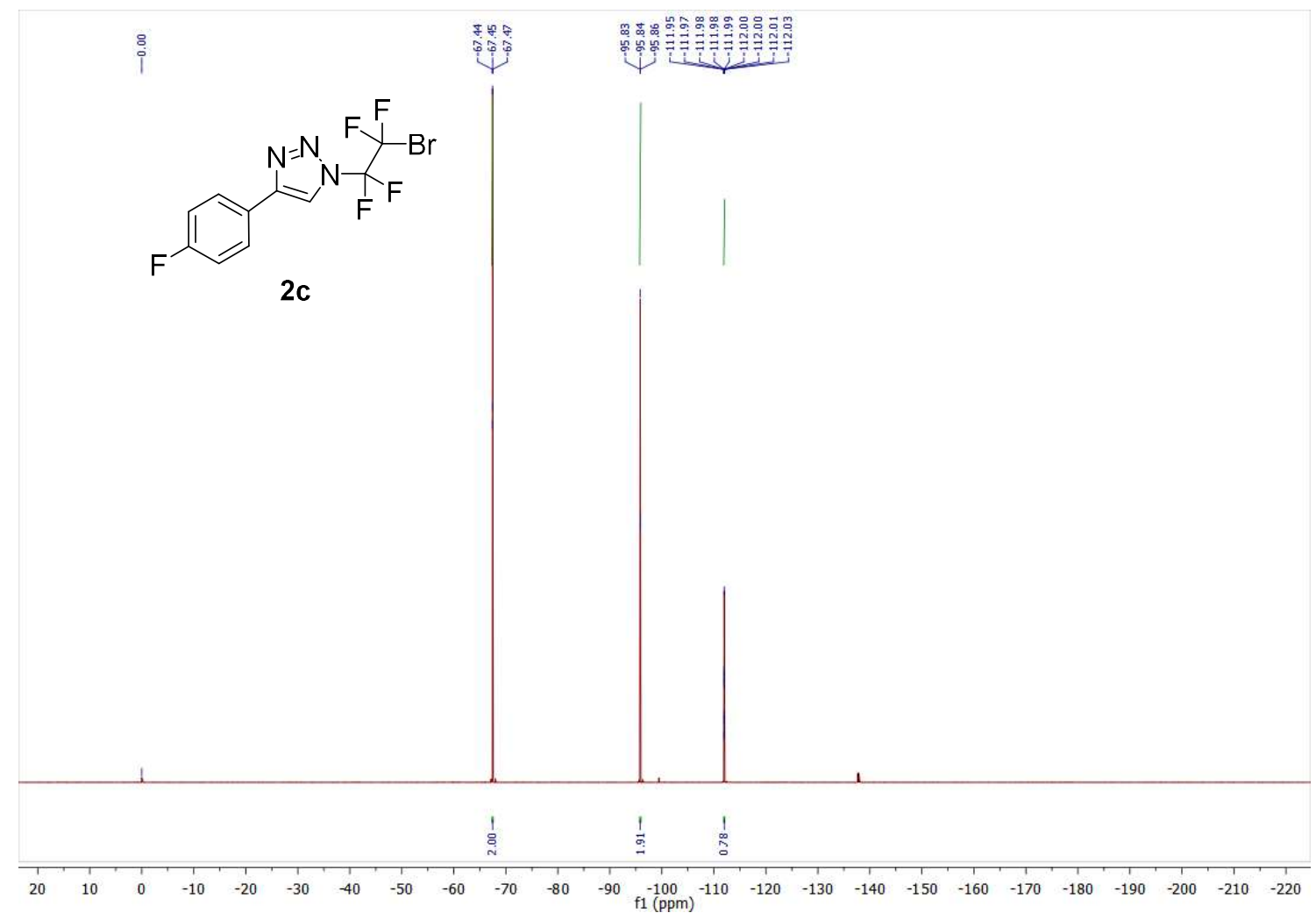

${ }^{1} \mathrm{H}$ NMR spectrum of $\mathbf{2 d}\left(\mathrm{CDCl}_{3}, 400 \mathrm{MHz}\right)$

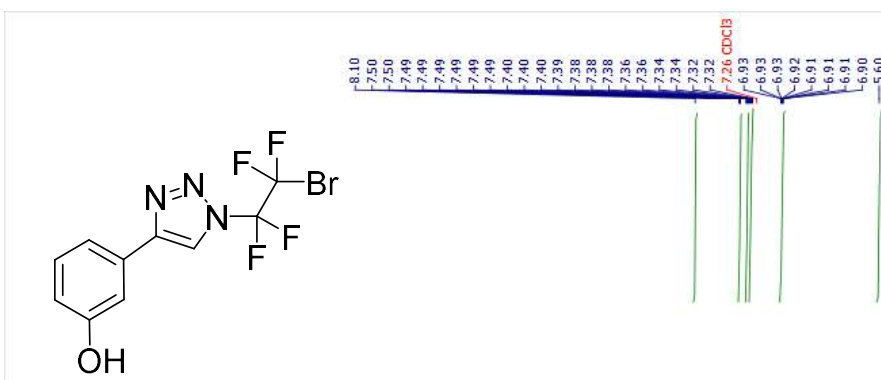

$2 d$

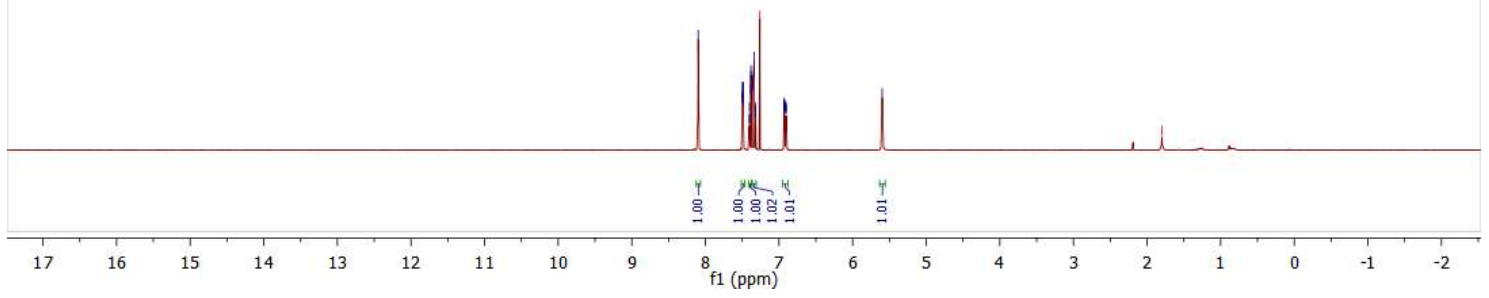


${ }^{13} \mathrm{C}\left\{{ }^{1} \mathrm{H}\right\}$ NMR spectrum of $\mathbf{2} \mathbf{d}\left(\mathrm{CDCl}_{3}, 101 \mathrm{MHz}\right)$
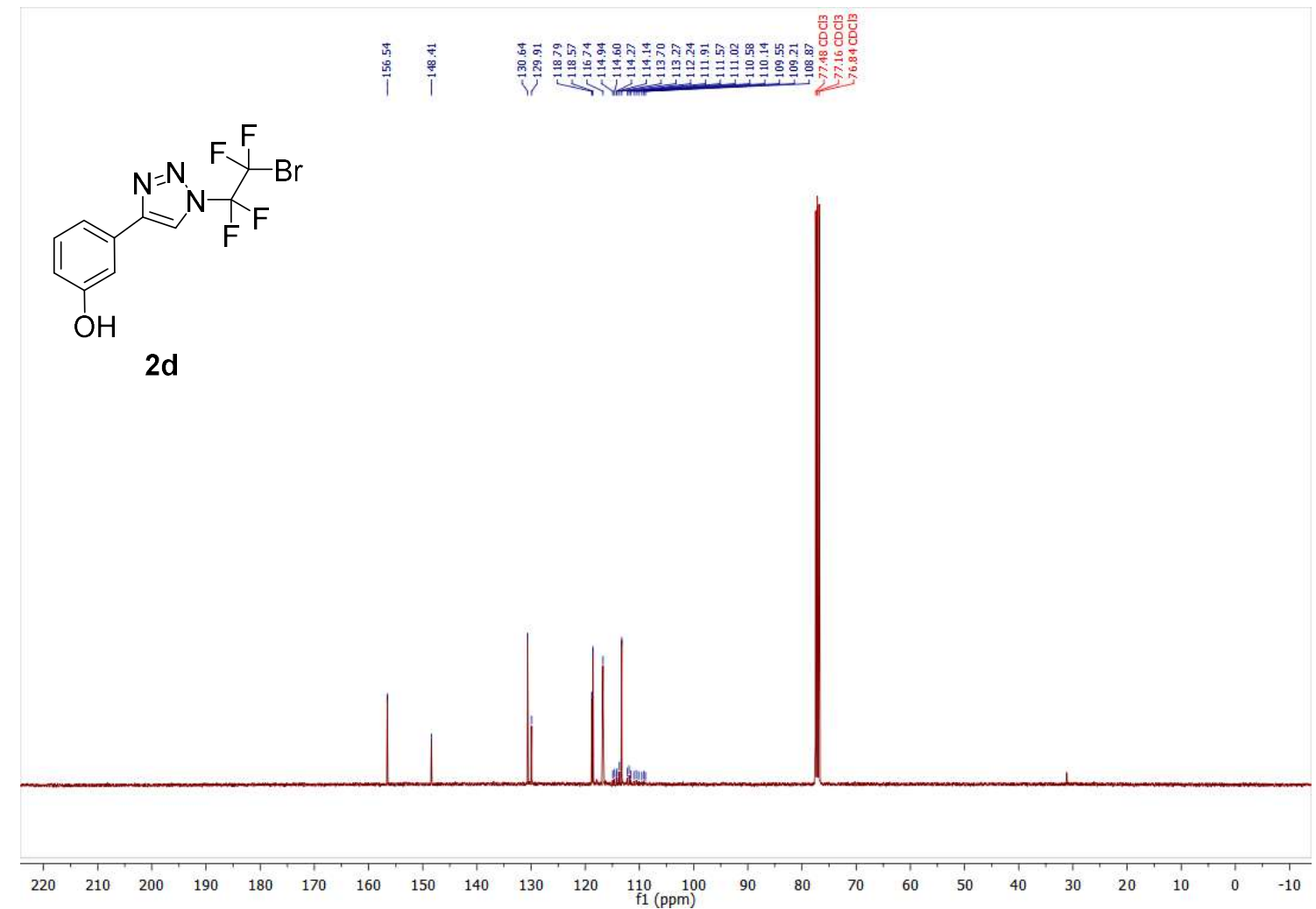

${ }^{19} \mathrm{~F}$ NMR spectrum of $\mathbf{2} \mathbf{d}\left(\mathrm{CDCl}_{3}, 377 \mathrm{MHz}\right)$

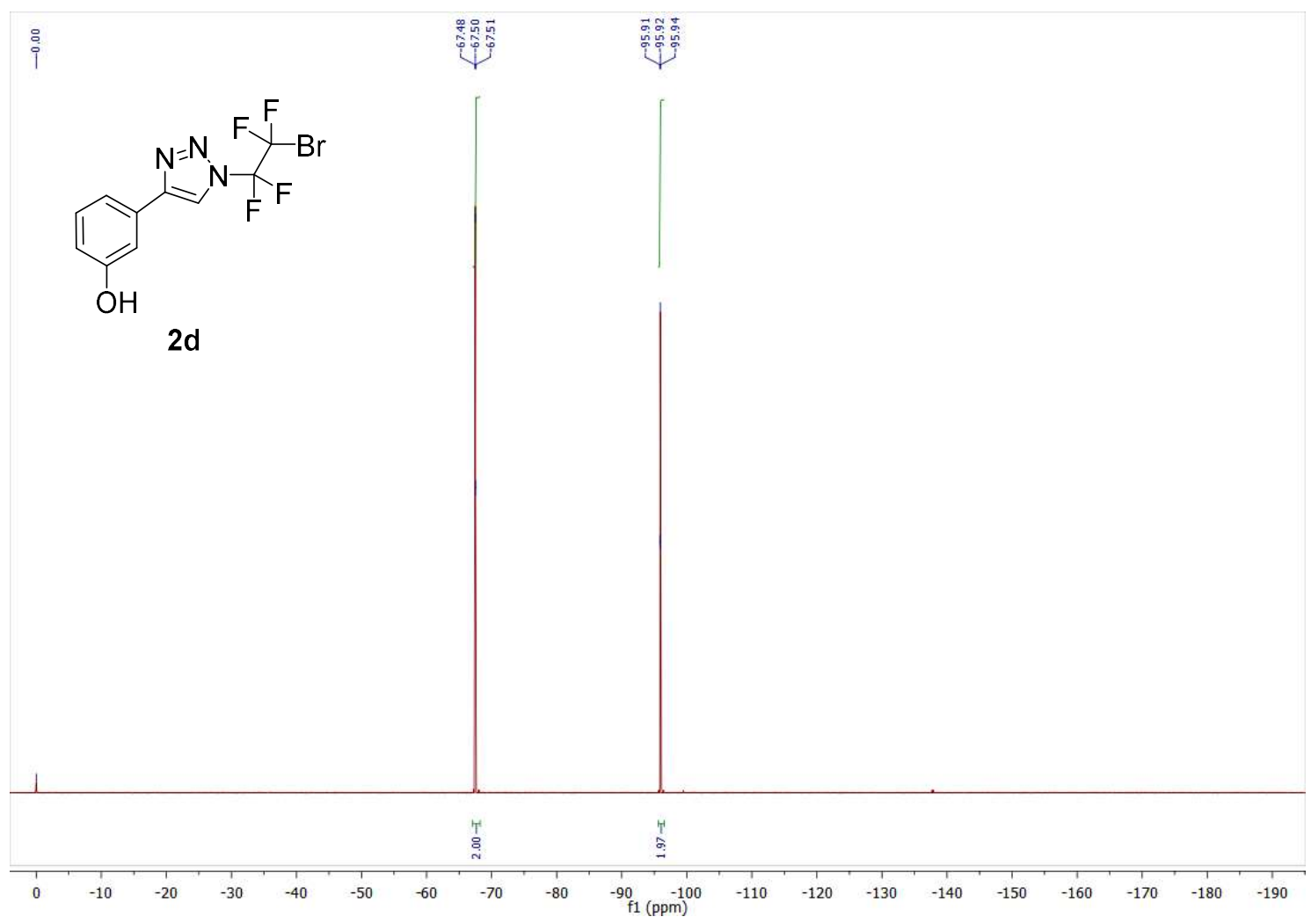


${ }^{1} \mathrm{H}$ NMR spectrum of $2 \mathbf{e}\left(\mathrm{CDCl}_{3}, 400 \mathrm{MHz}\right)$

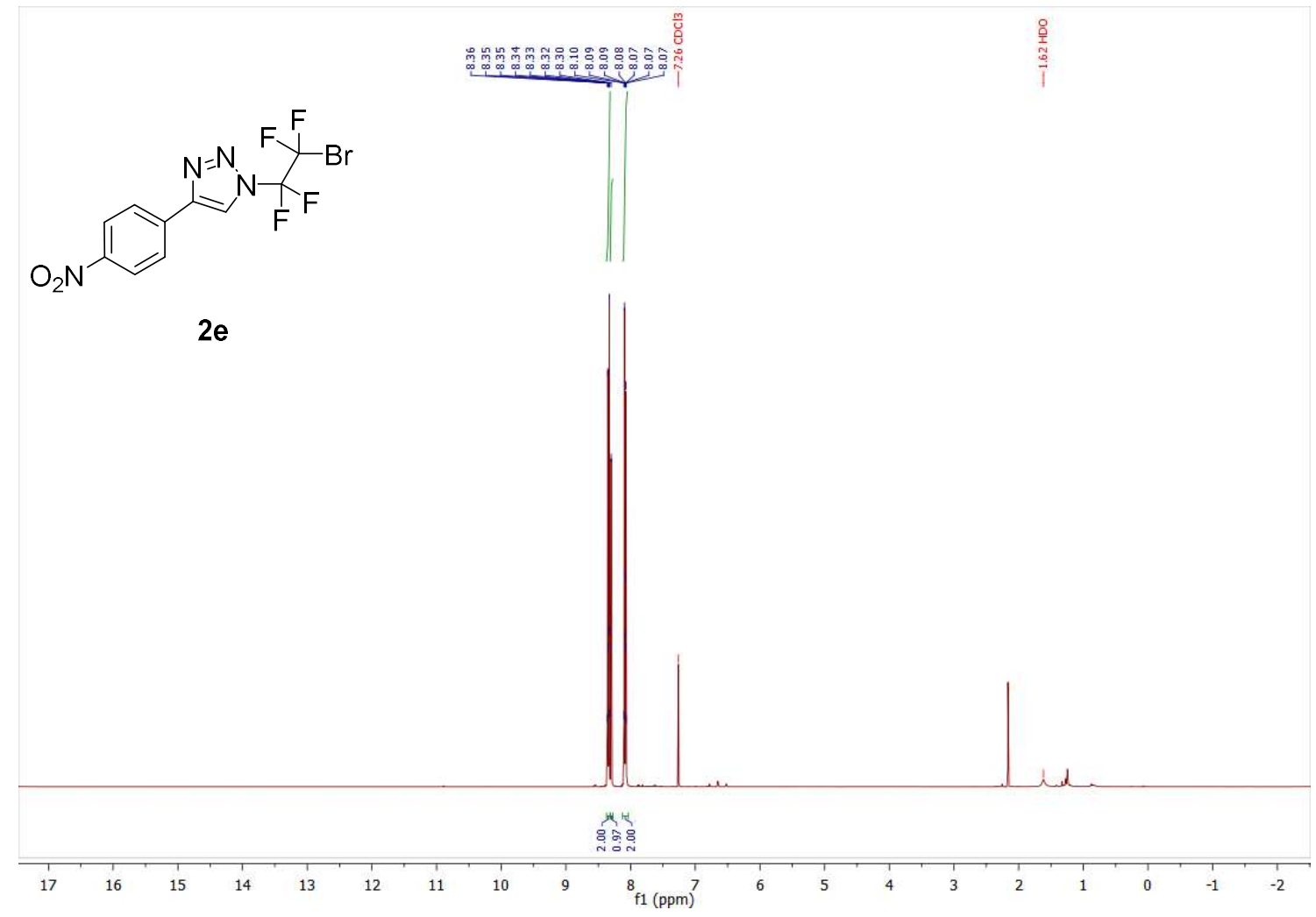

${ }^{13} \mathrm{C}\left\{{ }^{1} \mathrm{H}\right\}$ NMR spectrum of $2 \mathrm{e}\left(\mathrm{CDCl}_{3}, 101 \mathrm{MHz}\right)$

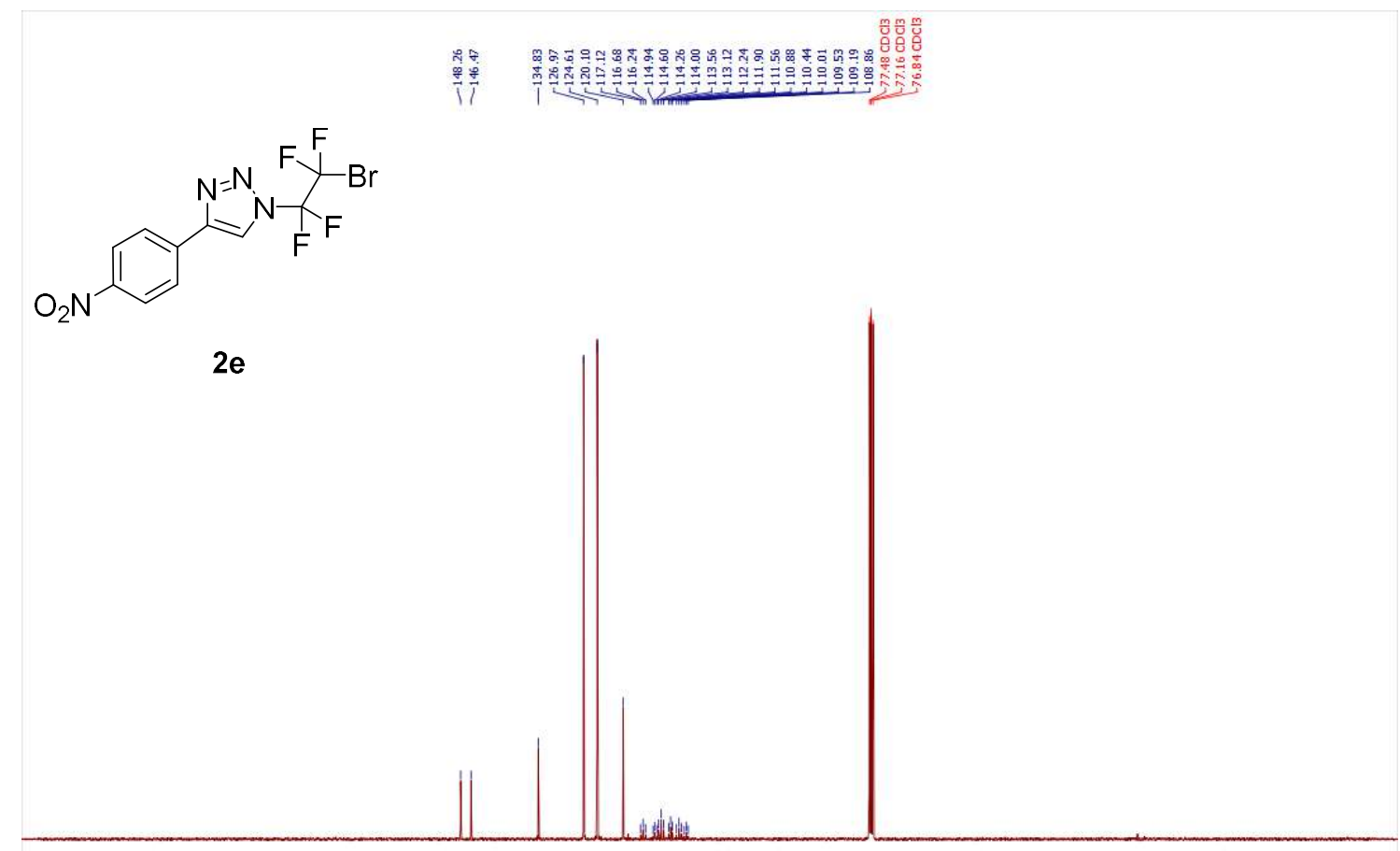

$\begin{array}{llllllllllllllllllllllllll}220 & 210 & 200 & 190 & 180 & 170 & 160 & 150 & 140 & 130 & 120 & \underset{\mathrm{f} 1(\mathrm{ppm})}{110} & 90 & 80 & 70 & 60 & 50 & 40 & 30 & 20 & 10 & 0 & -10\end{array}$ 
${ }^{19} \mathrm{~F}$ NMR spectrum of $\mathbf{2 e}\left(\mathrm{CDCl}_{3}, 377 \mathrm{MHz}\right)$
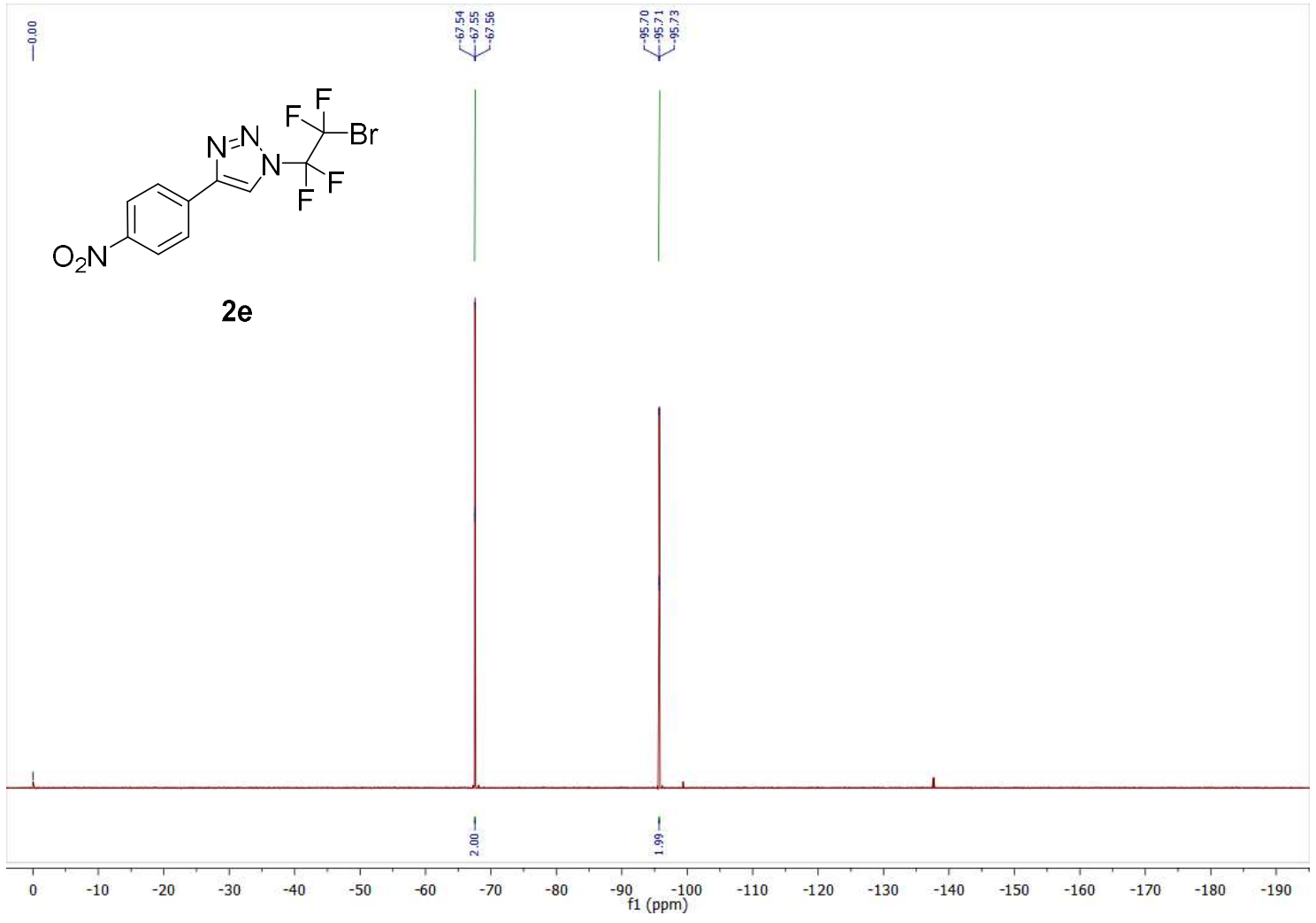

${ }^{1} \mathrm{H}$ NMR spectrum of $\mathbf{2 f}\left(\mathrm{CDCl}_{3}, 400 \mathrm{MHz}\right)$

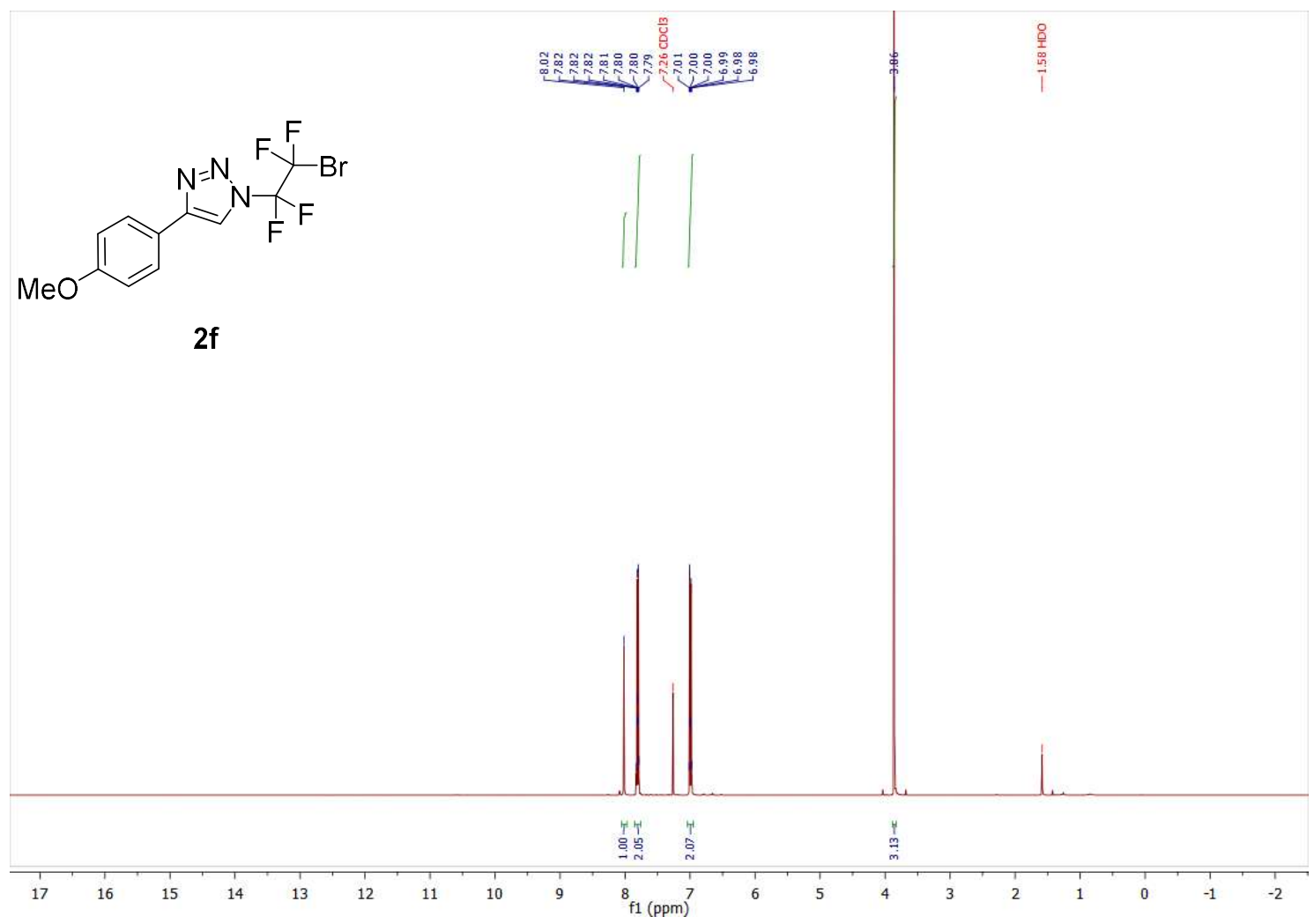


${ }^{13} \mathrm{C}\left\{{ }^{1} \mathrm{H}\right\}$ NMR spectrum of $\mathbf{2 f}\left(\mathrm{CDCl}_{3}, 101 \mathrm{MHz}\right)$
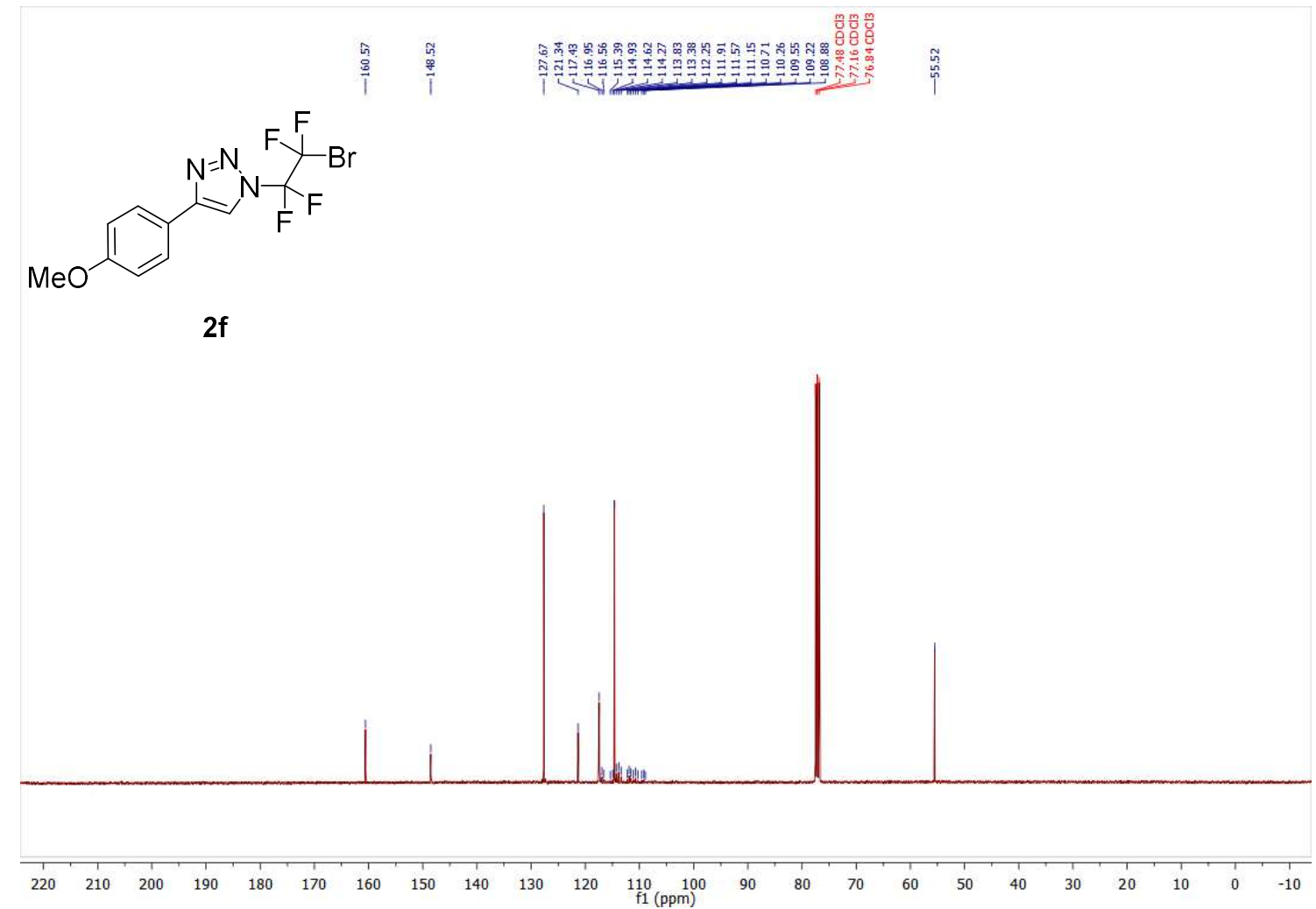

${ }^{19} \mathrm{~F}$ NMR spectrum of $\mathbf{2 f}\left(\mathrm{CDCl}_{3}, 377 \mathrm{MHz}\right)$

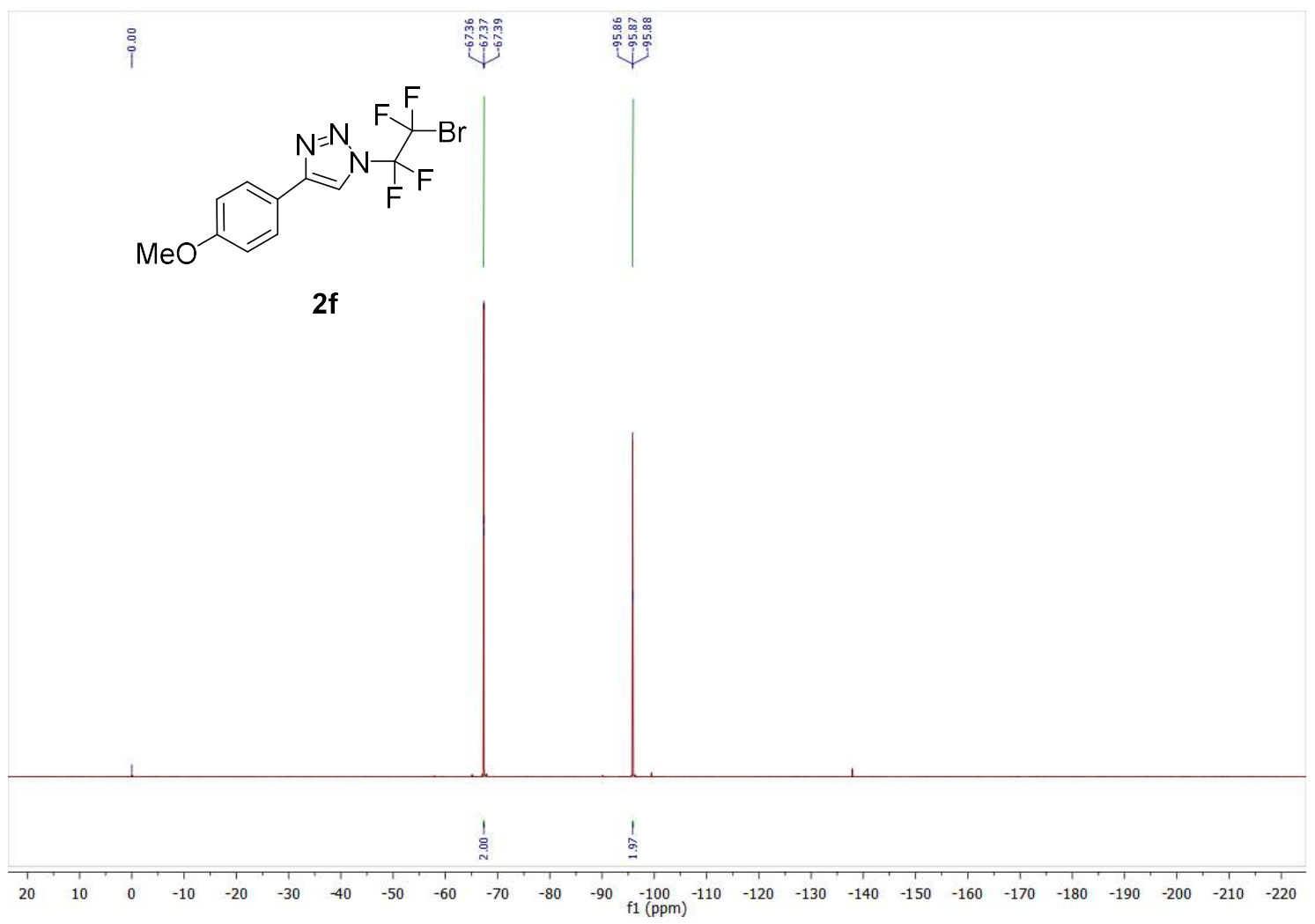


${ }^{1} \mathrm{H}$ NMR spectrum of $\mathbf{2} \mathbf{g}\left(\mathrm{CDCl}_{3}, 400 \mathrm{MHz}\right)$

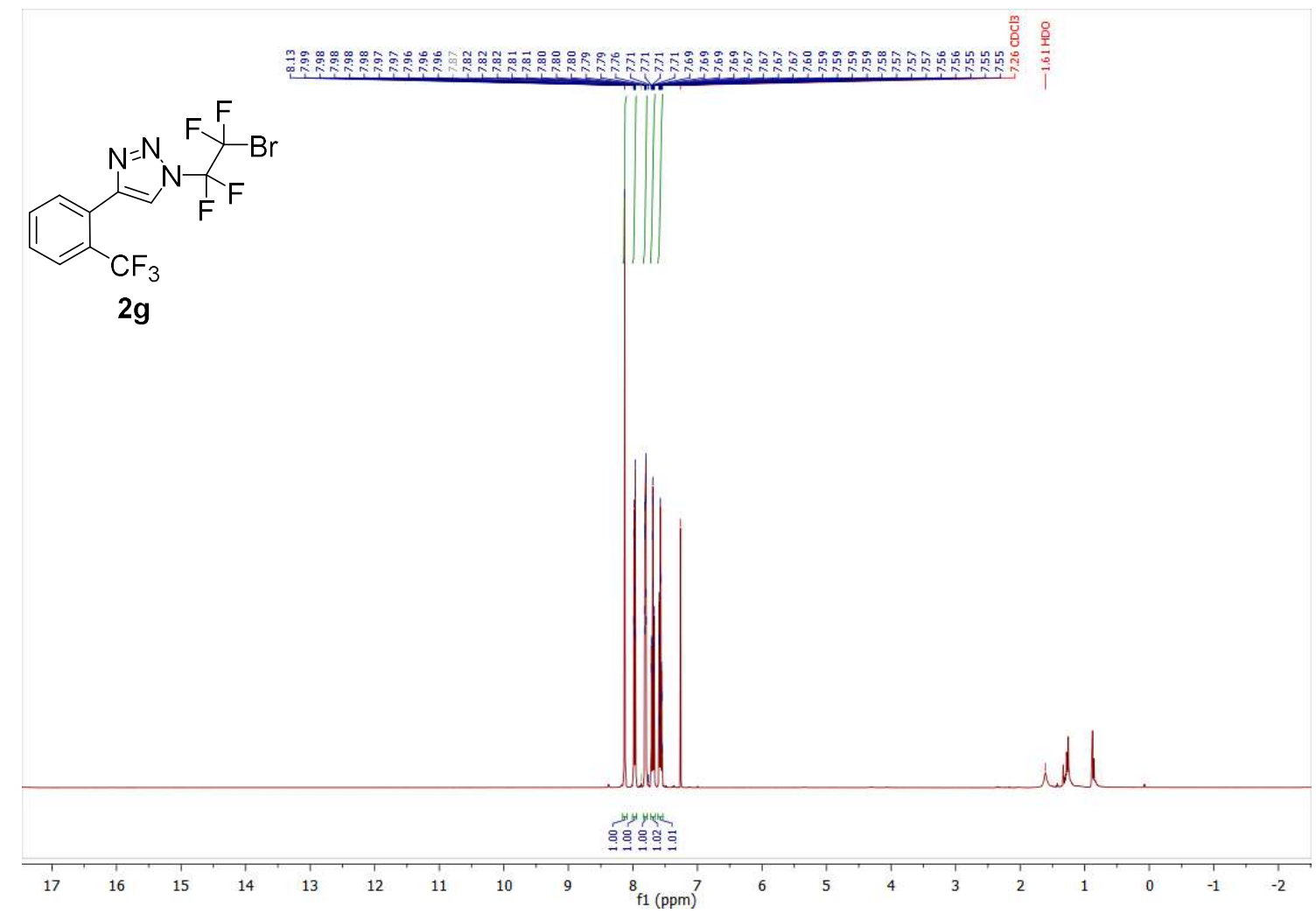

${ }^{13} \mathrm{C}\left\{{ }^{1} \mathrm{H}\right\}$ NMR spectrum of $\mathbf{2 g}\left(\mathrm{CDCl}_{3}, 101 \mathrm{MHz}\right)$

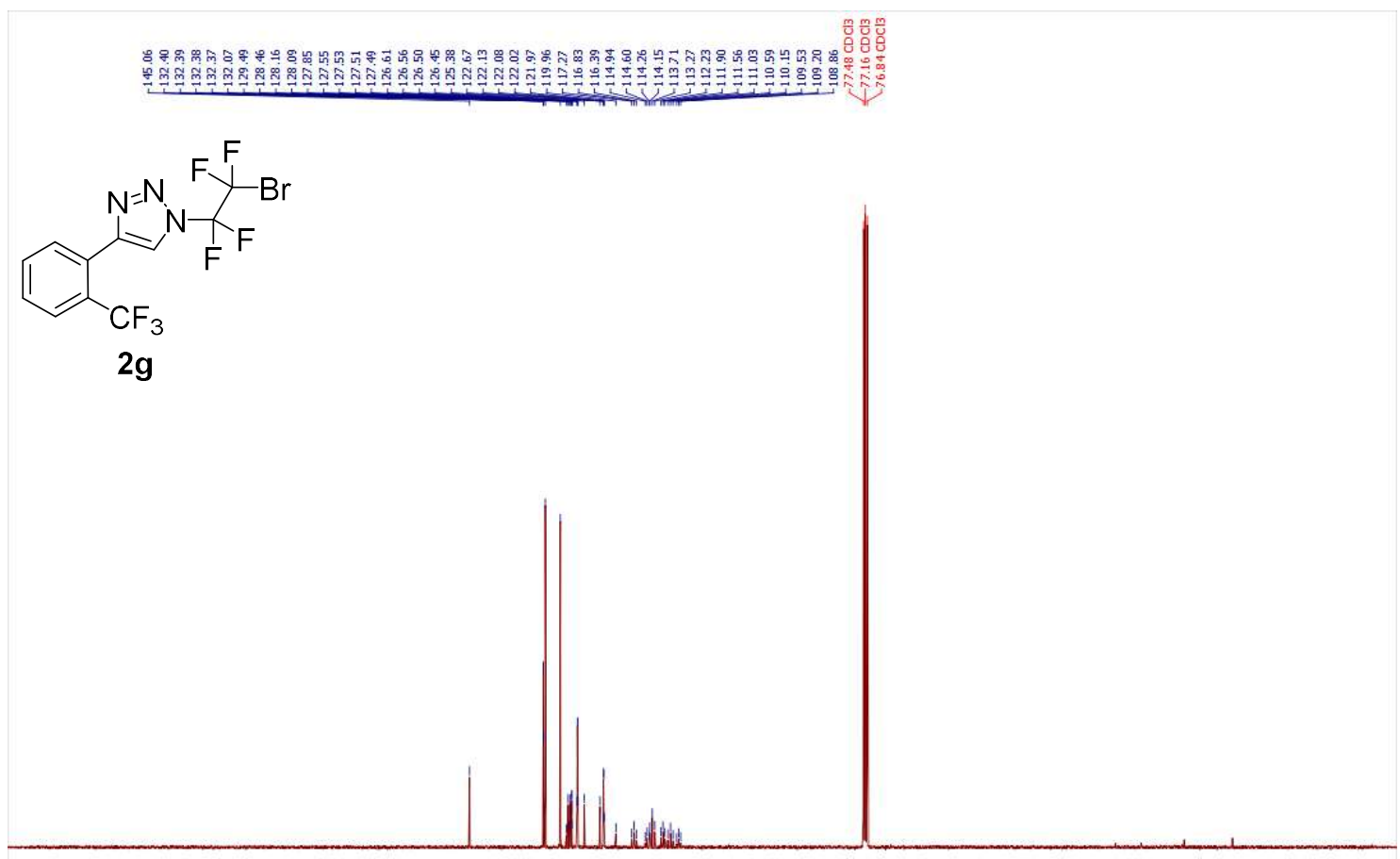

$\begin{array}{llllllllllllllllllllllll}220 & 210 & 200 & 190 & 180 & 170 & 160 & 150 & 140 & 130 & 120 & \underset{\mathrm{f} 1(\mathrm{ppm})}{110} & 90 & 80 & 70 & 60 & 50 & 40 & 30 & 20 & 10 & 0 & -10\end{array}$ 
${ }^{19} \mathrm{~F}$ NMR spectrum of $\mathbf{2 g}\left(\mathrm{CDCl}_{3}, 377 \mathrm{MHz}\right)$

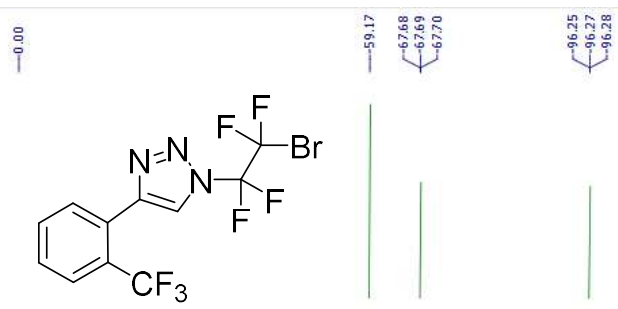

$2 \mathrm{~g}$

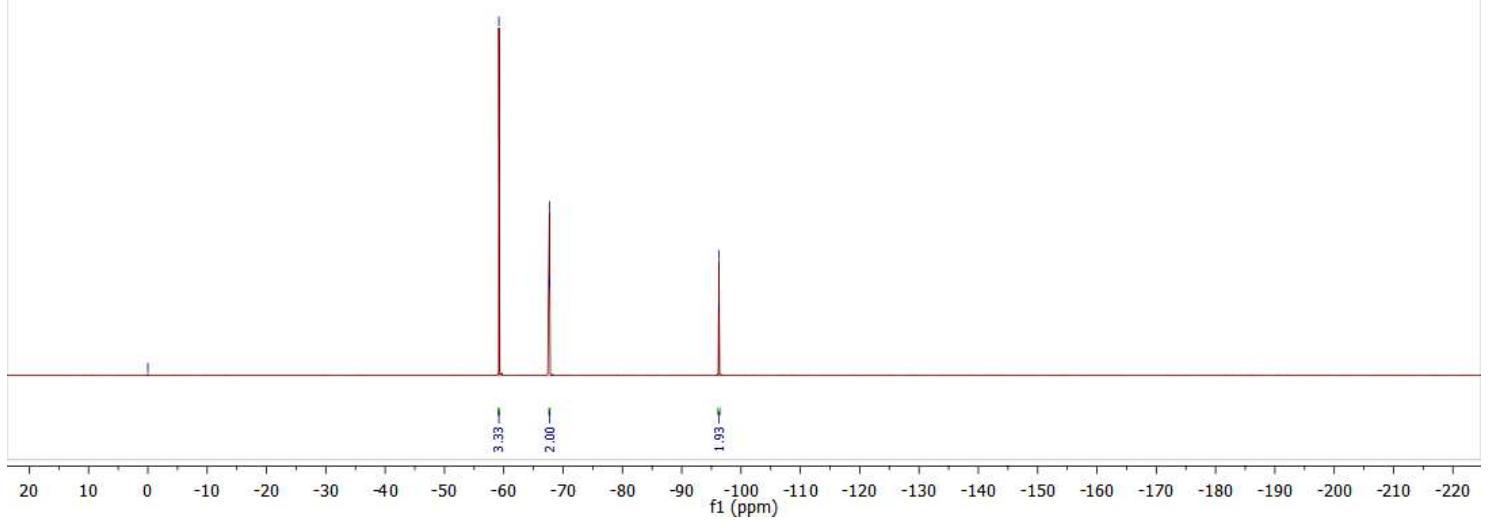

${ }^{1} \mathrm{H}$ NMR spectrum of $\mathbf{2} \mathbf{h}\left(\mathrm{CDCl}_{3}, 400 \mathrm{MHz}\right)$

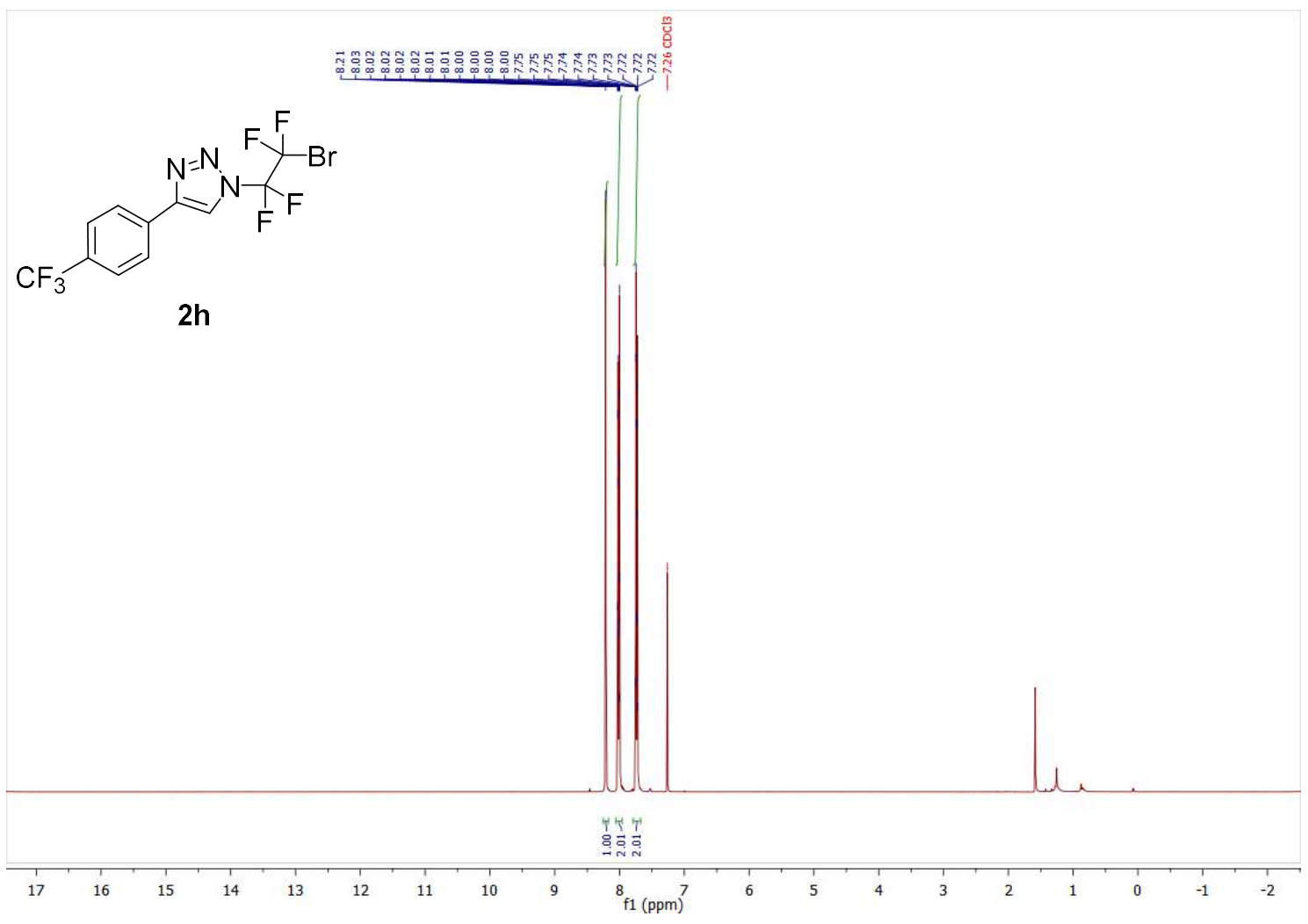


${ }^{13} \mathrm{C}\left\{{ }^{1} \mathrm{H}\right\}$ NMR spectrum of $\mathbf{2} \mathbf{h}\left(\mathrm{CDCl}_{3}, 101 \mathrm{MHz}\right)$
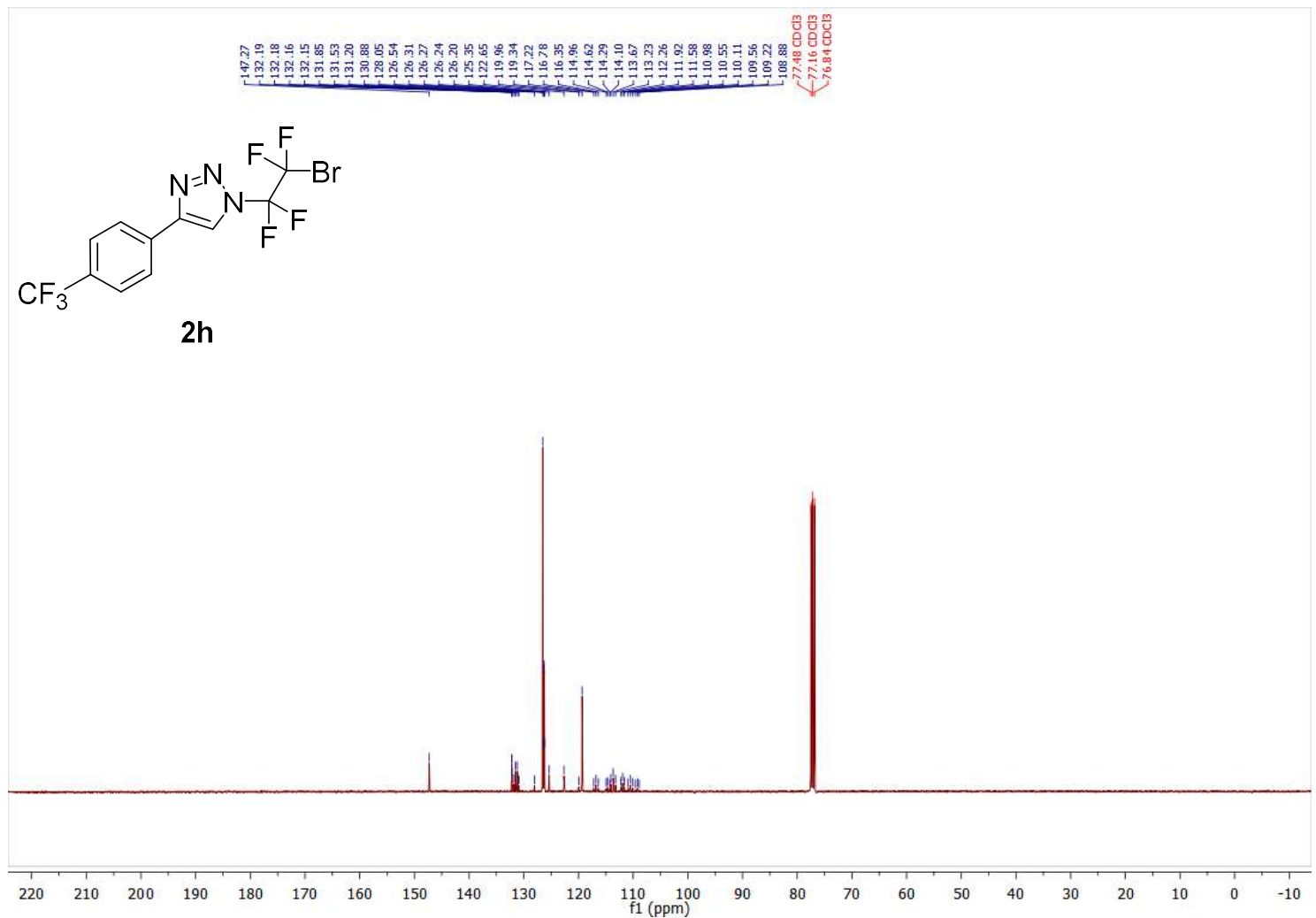

${ }^{19} \mathrm{~F}$ NMR spectrum of $\mathbf{2} \mathbf{h}\left(\mathrm{CDCl}_{3}, 377 \mathrm{MHz}\right)$

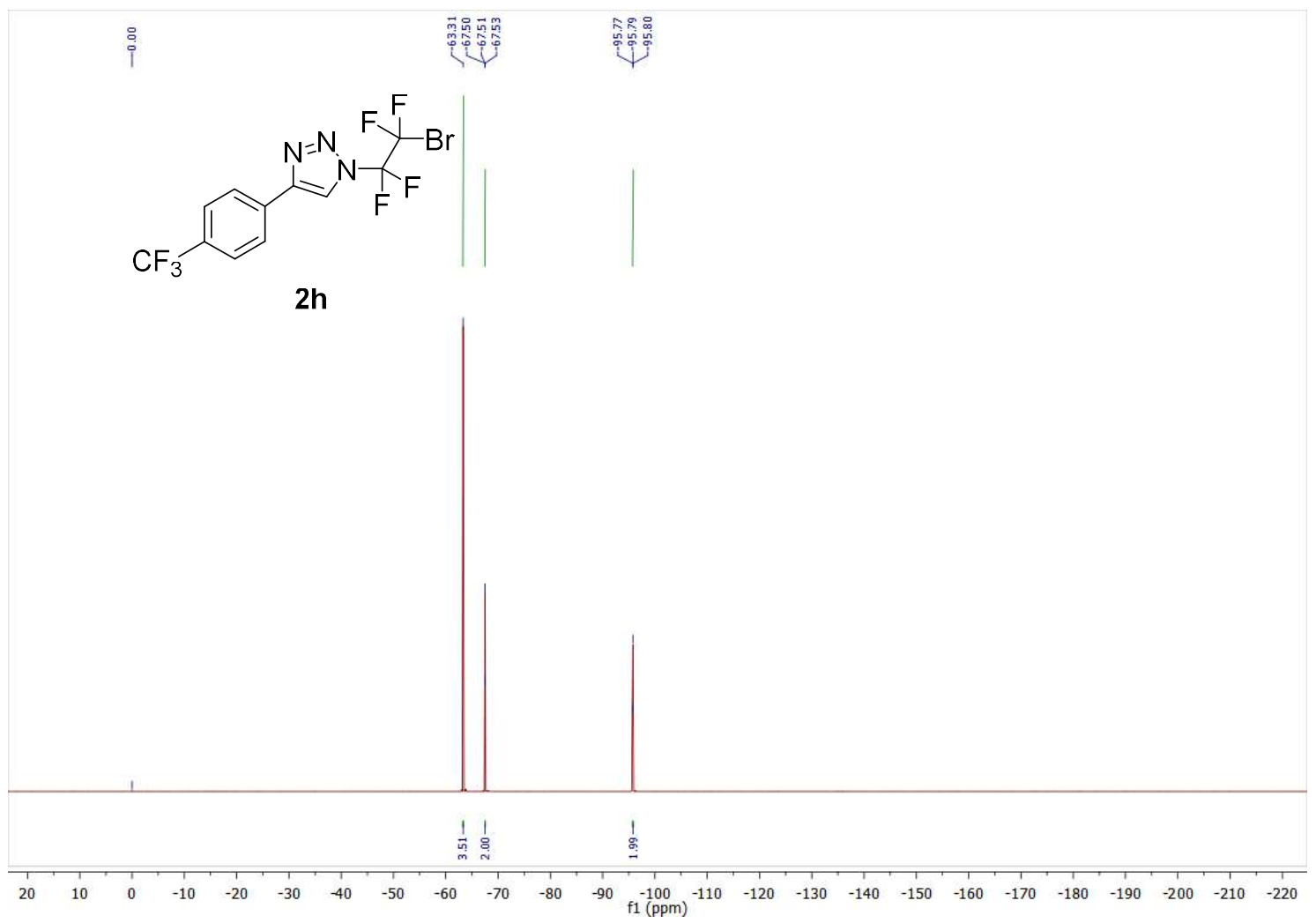


${ }^{1} \mathrm{H}$ NMR spectrum of $\mathbf{2 i}\left(\mathrm{CDCl}_{3}, 400 \mathrm{MHz}\right)$

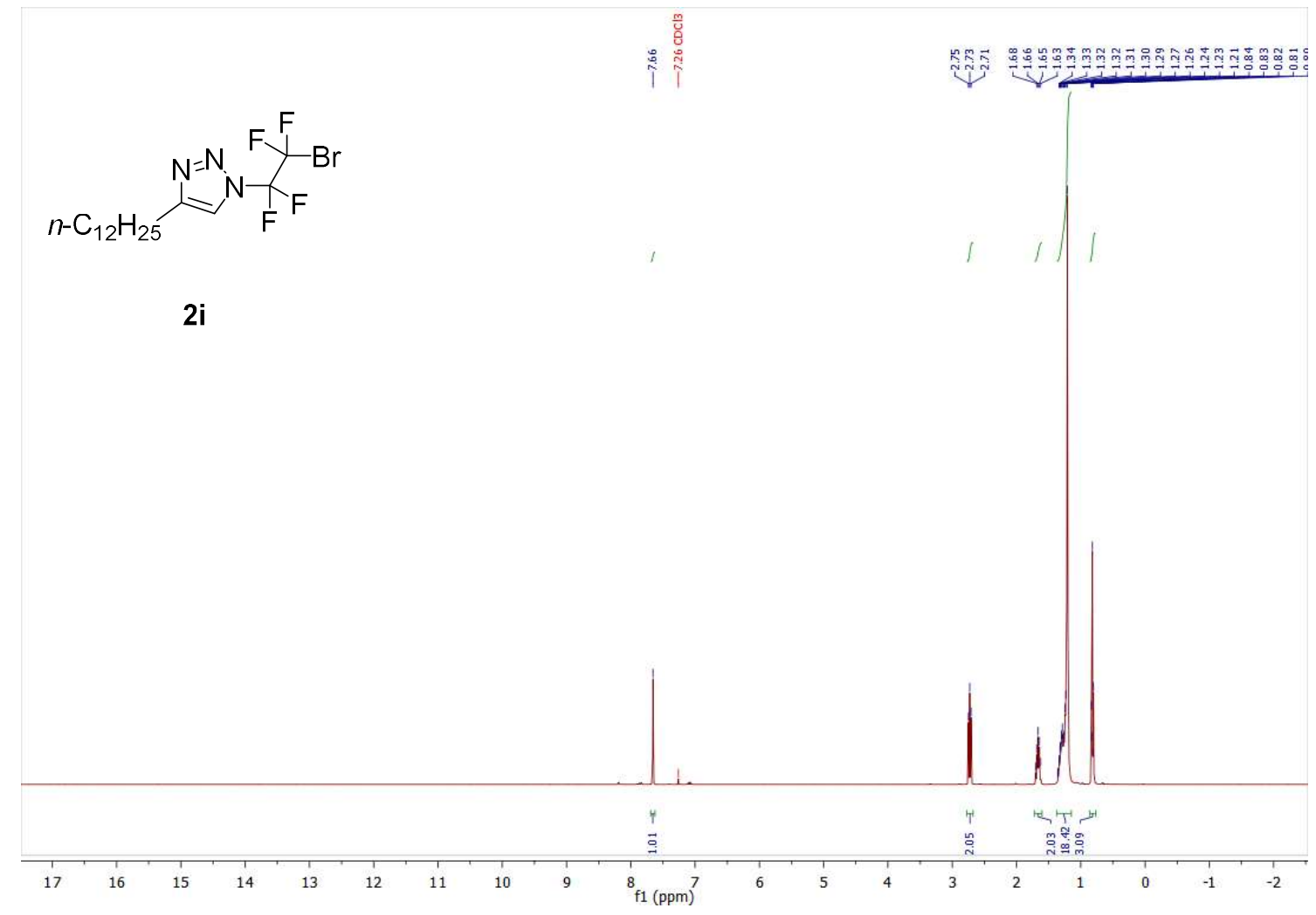

${ }^{13} \mathrm{C}\left\{{ }^{1} \mathrm{H}\right\}$ NMR spectrum of $\mathbf{2 i}\left(\mathrm{CDCl}_{3}, 101 \mathrm{MHz}\right)$

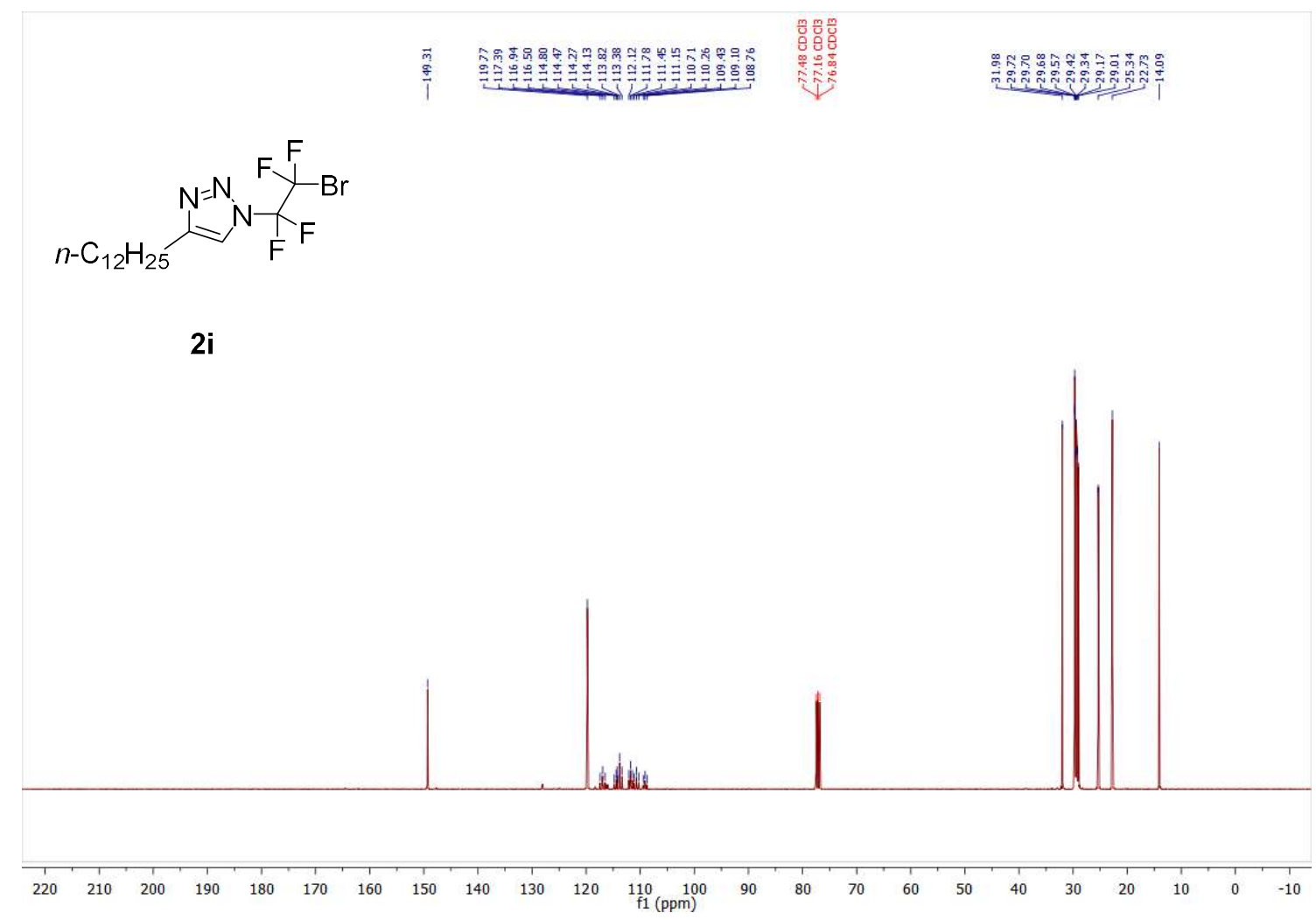


${ }^{19} \mathrm{~F}$ NMR spectrum of $2 \mathbf{i}\left(\mathrm{CDCl}_{3}, 377 \mathrm{MHz}\right)$
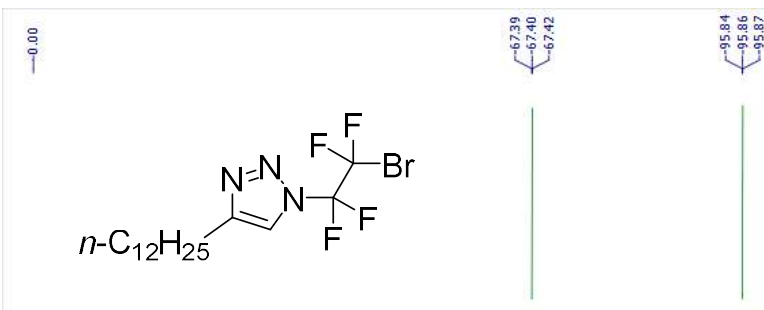

$2 \mathrm{i}$

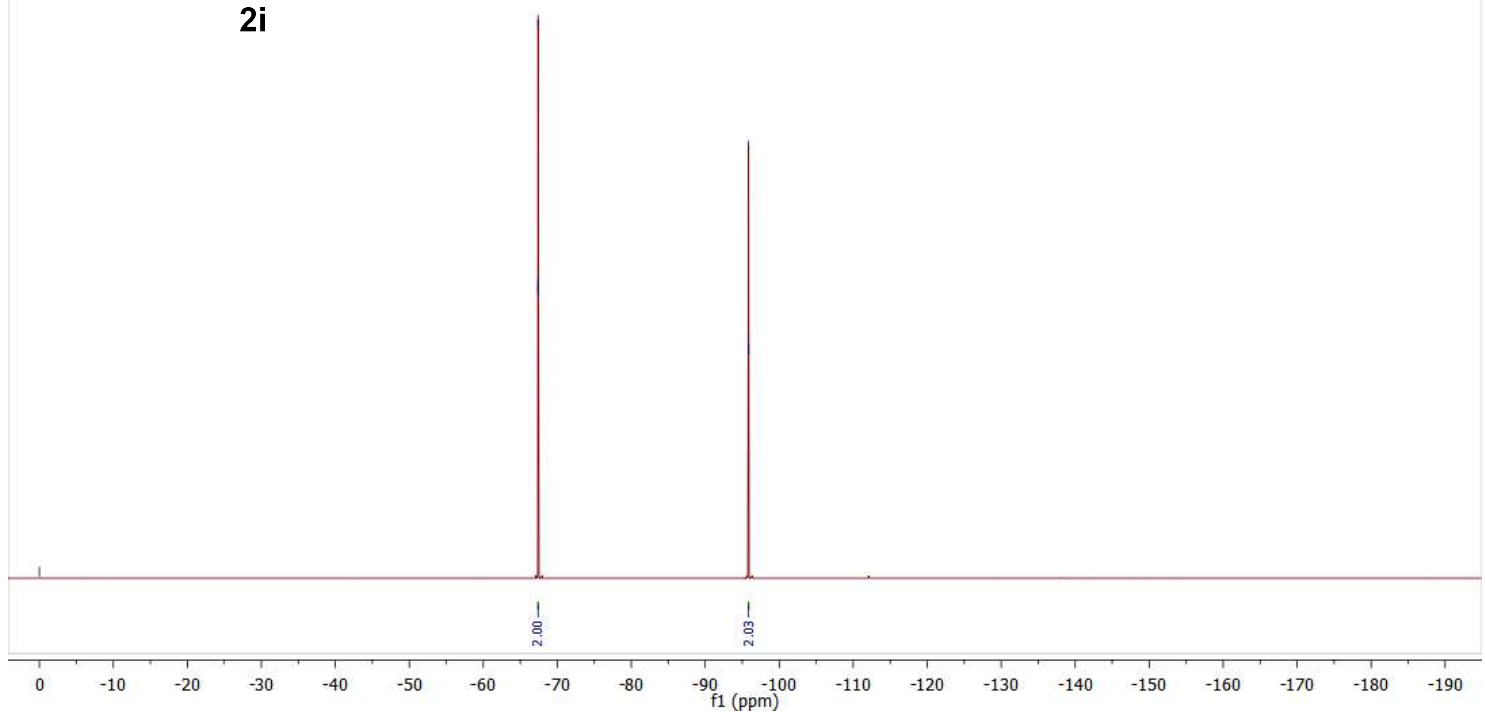

${ }^{1} \mathrm{H}$ NMR spectrum of $3 \mathrm{a}\left(\mathrm{CDCl}_{3}, 400 \mathrm{MHz}\right)$

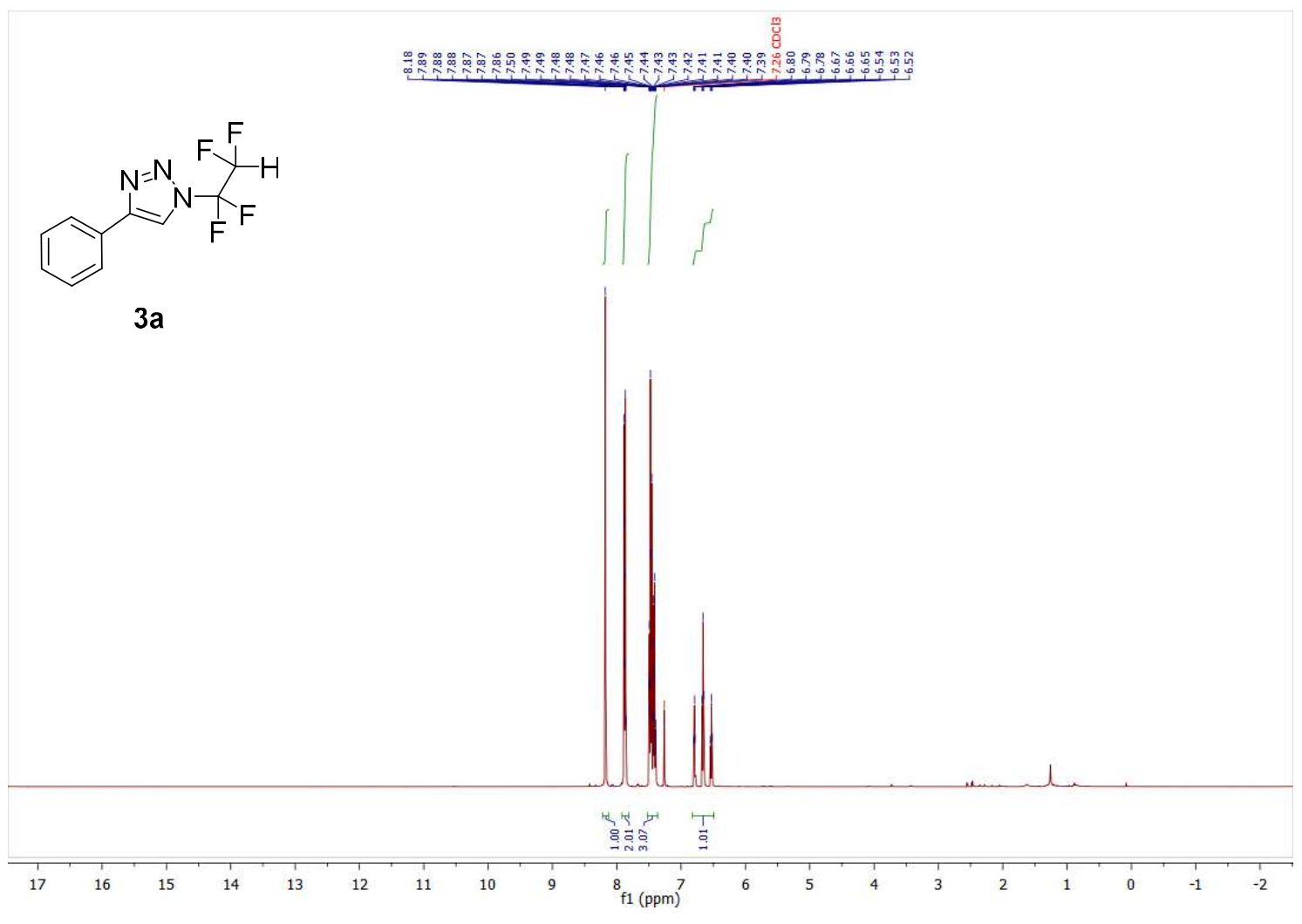


${ }^{13} \mathrm{C}\left\{{ }^{1} \mathrm{H}\right\}$ NMR spectrum of $\mathbf{3 a}\left(\mathrm{CDCl}_{3}, 101 \mathrm{MHz}\right)$
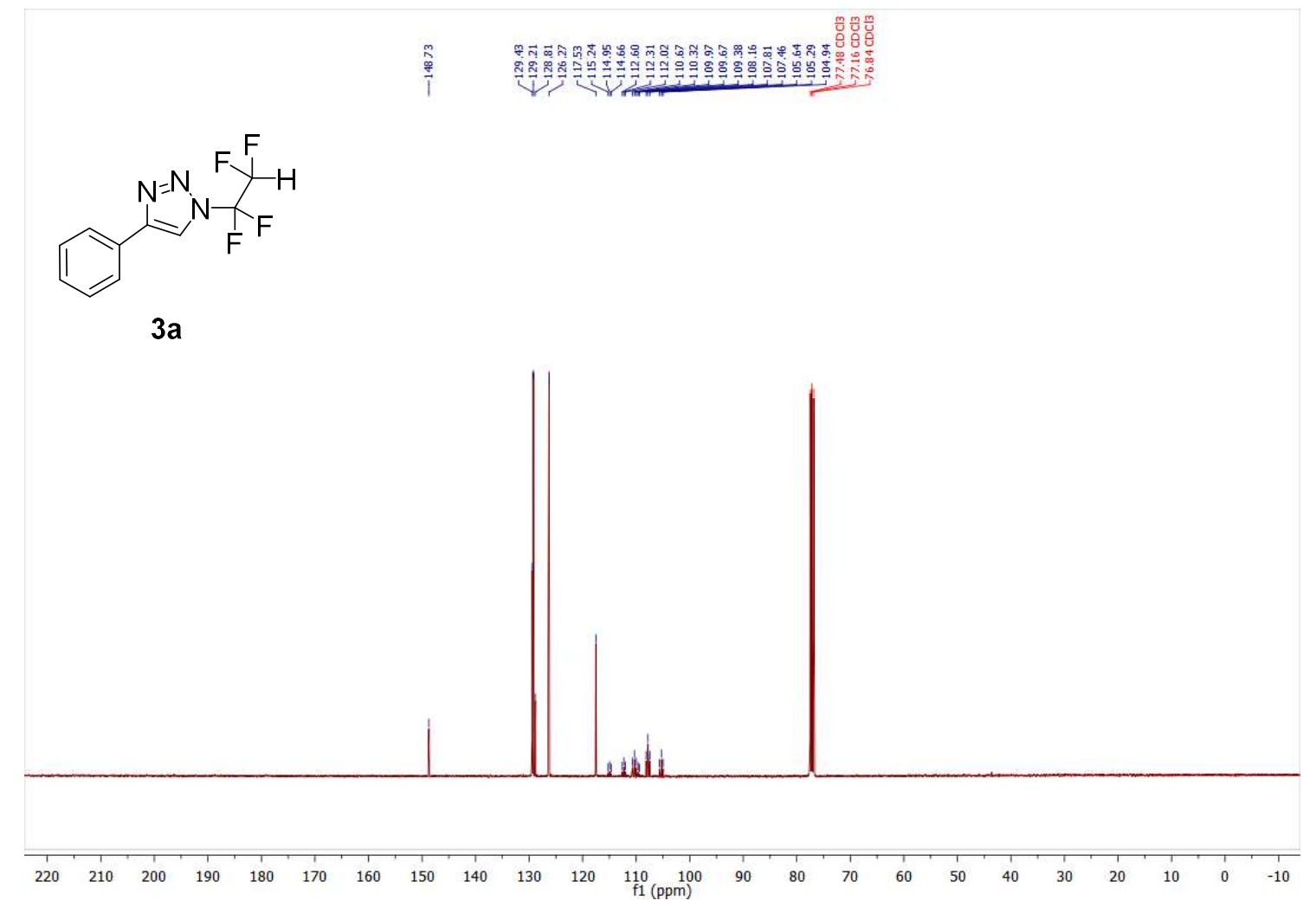

${ }^{19} \mathrm{~F}$ NMR spectrum of $\mathbf{3 a}\left(\mathrm{CDCl}_{3}, 377 \mathrm{MHz}\right)$

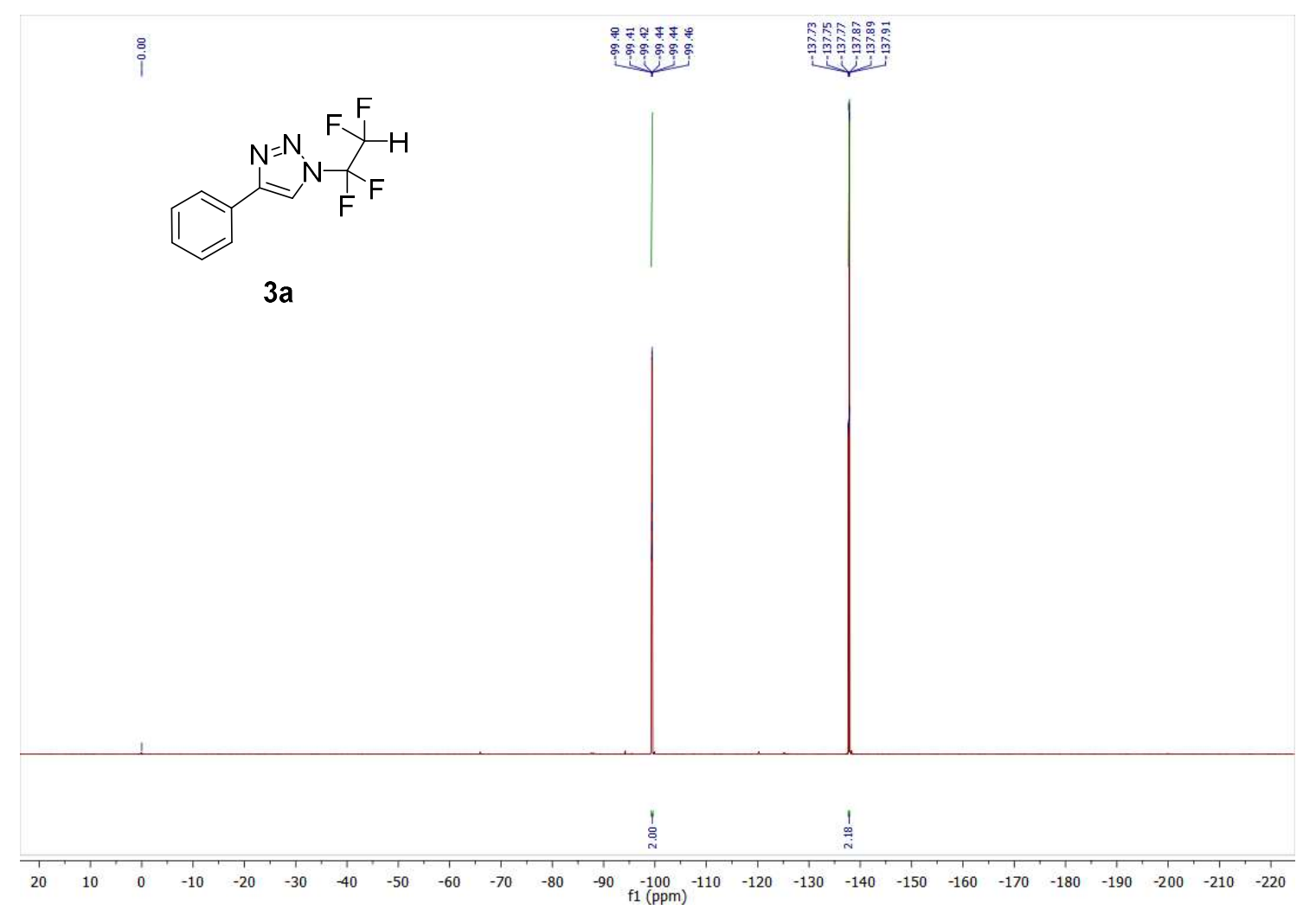


${ }^{1} \mathrm{H}$ NMR spectrum of $\mathbf{3} \mathbf{b}\left(\mathrm{CDCl}_{3}, 400 \mathrm{MHz}\right)$

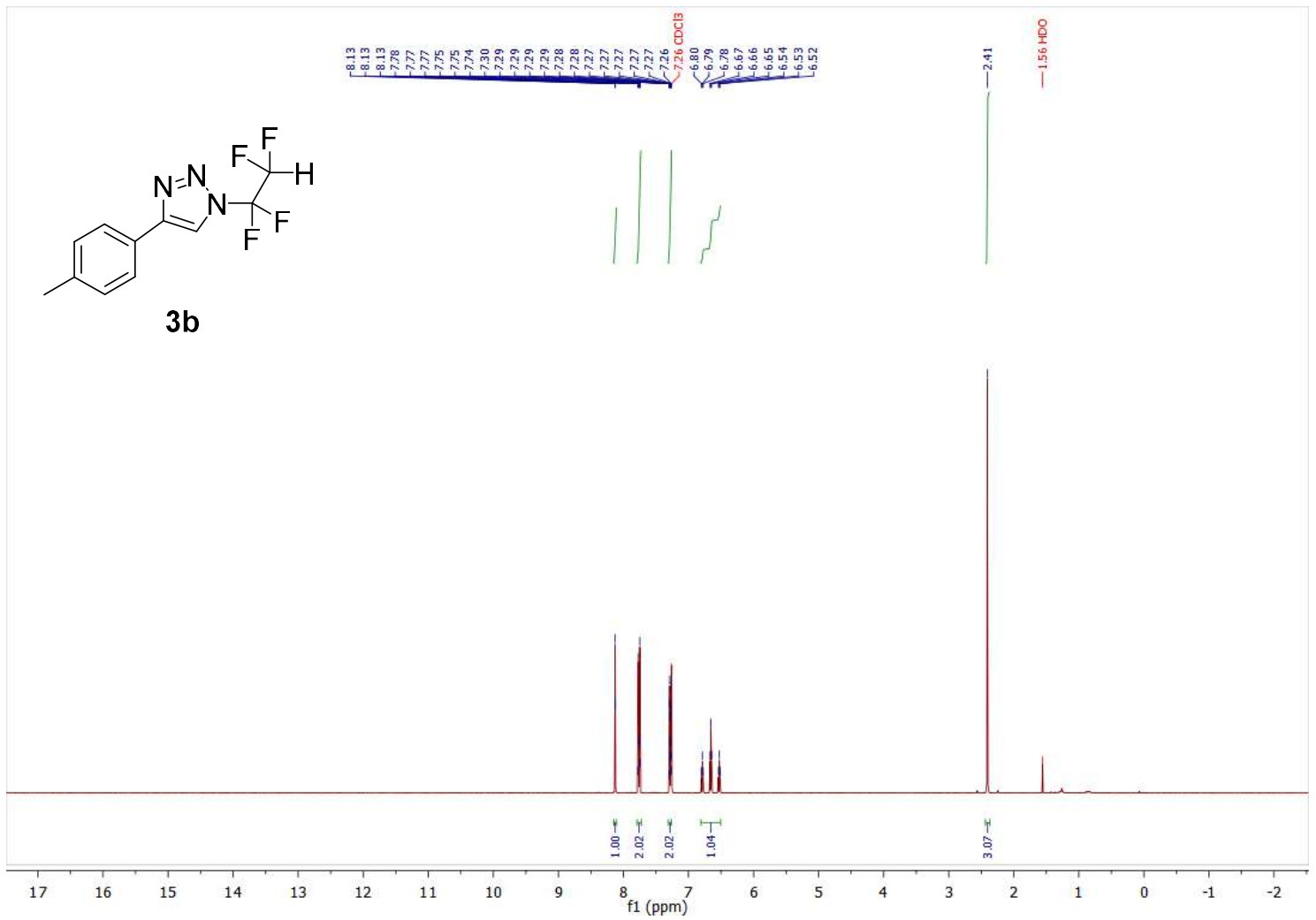

${ }^{19} \mathrm{~F}$ NMR spectrum of $\mathbf{3} \mathbf{b}\left(\mathrm{CDCl}_{3}, 377 \mathrm{MHz}\right)$

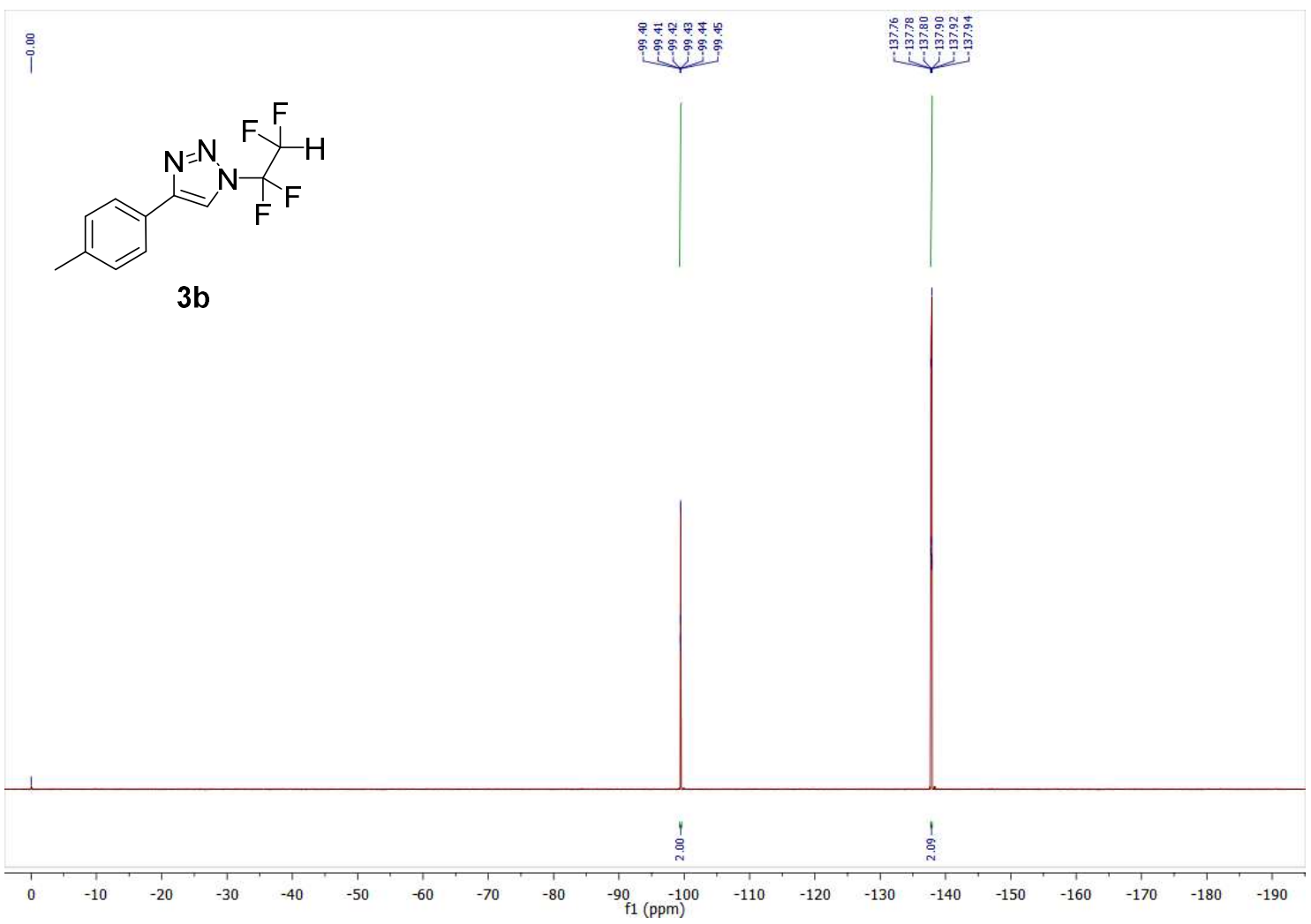


${ }^{1} \mathrm{H}$ NMR spectrum of $3 \mathrm{c}\left(\mathrm{CDCl}_{3}, 400 \mathrm{MHz}\right)$

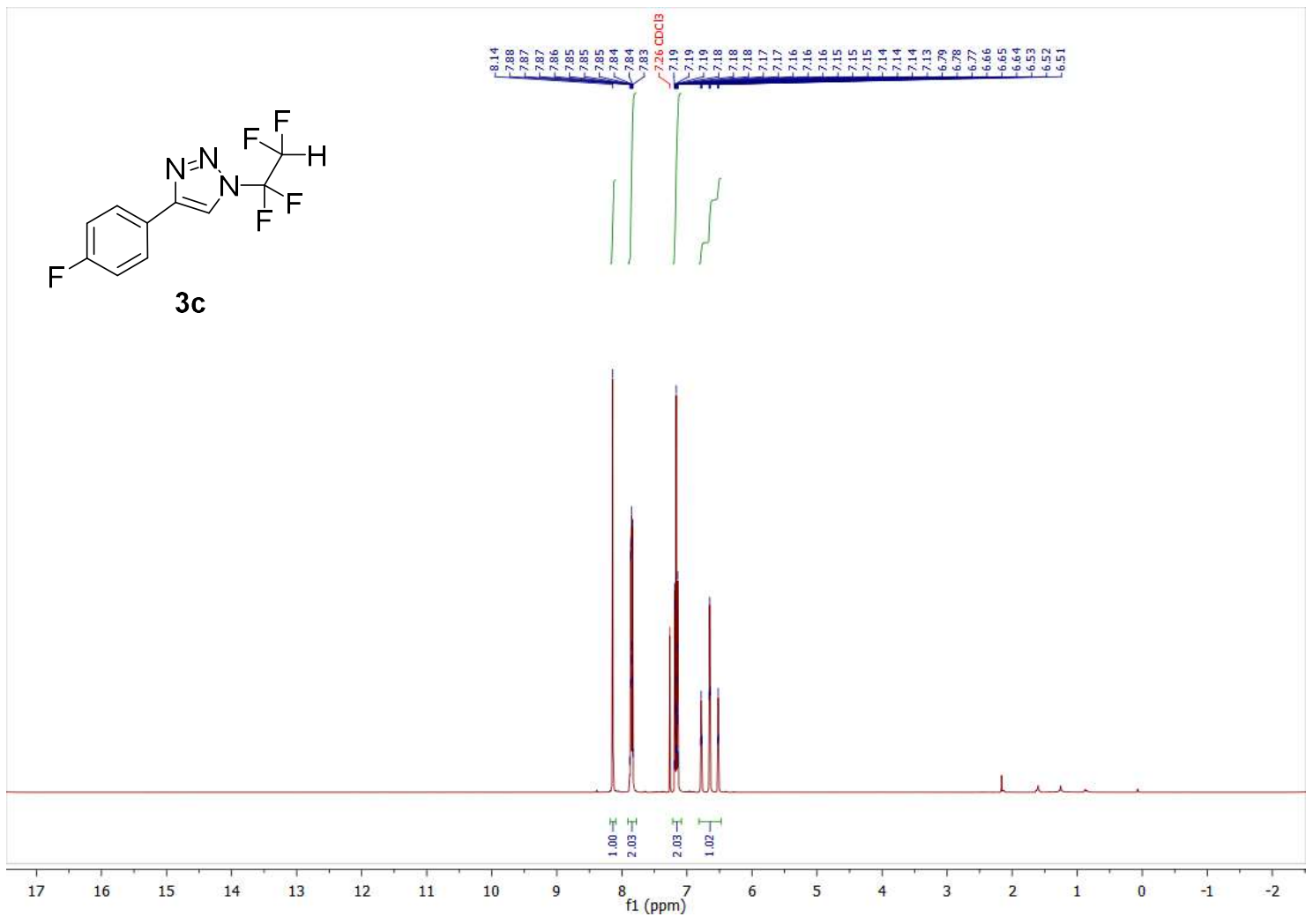

${ }^{13} \mathrm{C}\left\{{ }^{1} \mathrm{H}\right\}$ NMR spectrum of $3 \mathrm{c}\left(\mathrm{CDCl}_{3}, 101 \mathrm{MHz}\right)$

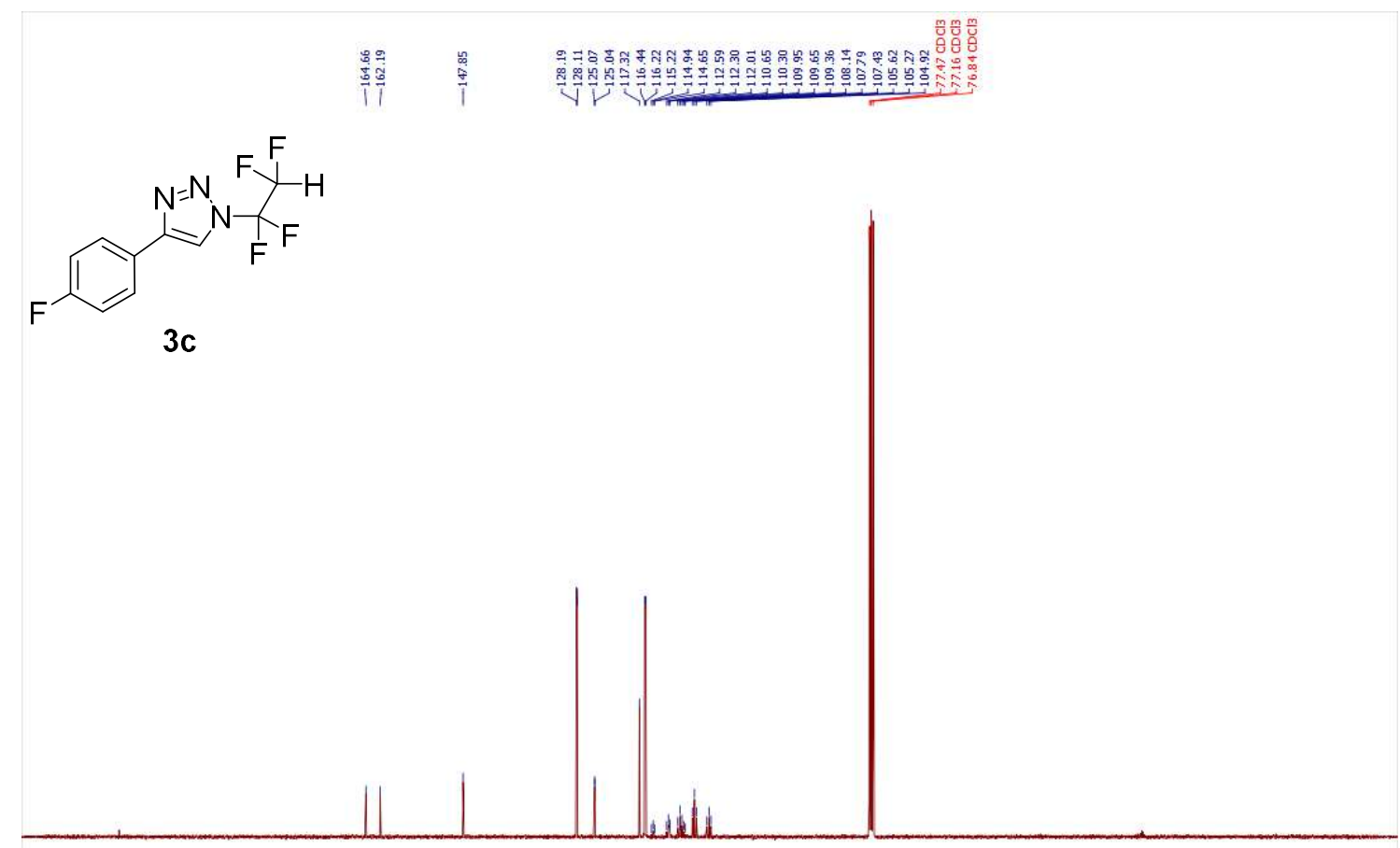

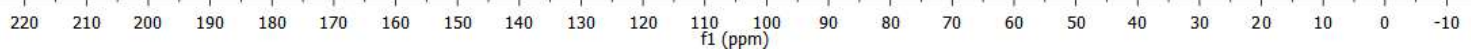


${ }^{19} \mathrm{~F}$ NMR spectrum of $3 \mathrm{c}\left(\mathrm{CDCl}_{3}, 377 \mathrm{MHz}\right)$

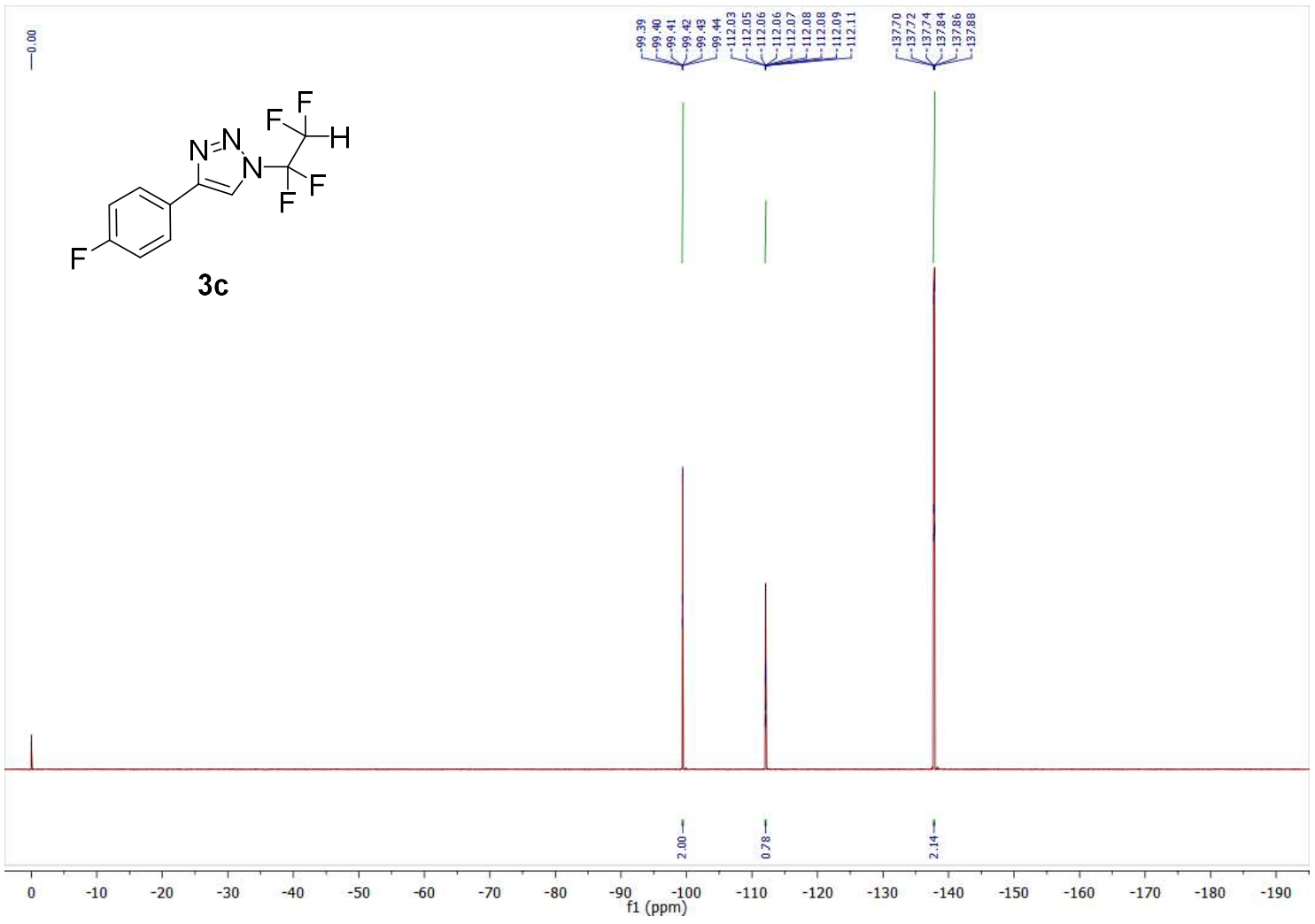

${ }^{1} \mathrm{H}$ NMR spectrum of $\mathbf{3 d}\left(\mathrm{CDCl}_{3}, 400 \mathrm{MHz}\right)$

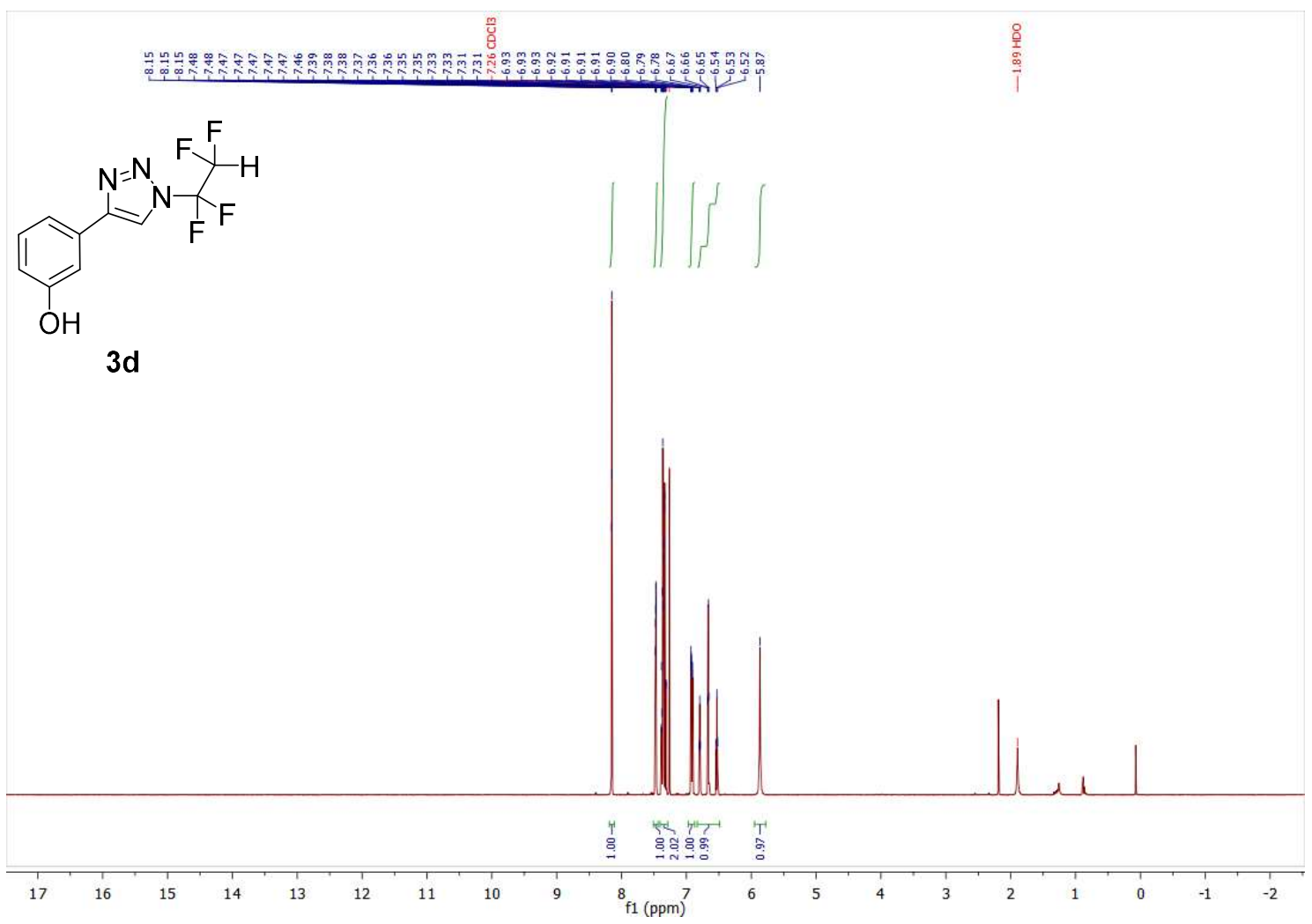


${ }^{13} \mathrm{C}\left\{{ }^{1} \mathrm{H}\right\}$ NMR spectrum of $\mathbf{3 d}\left(\mathrm{CDCl}_{3}, 101 \mathrm{MHz}\right)$
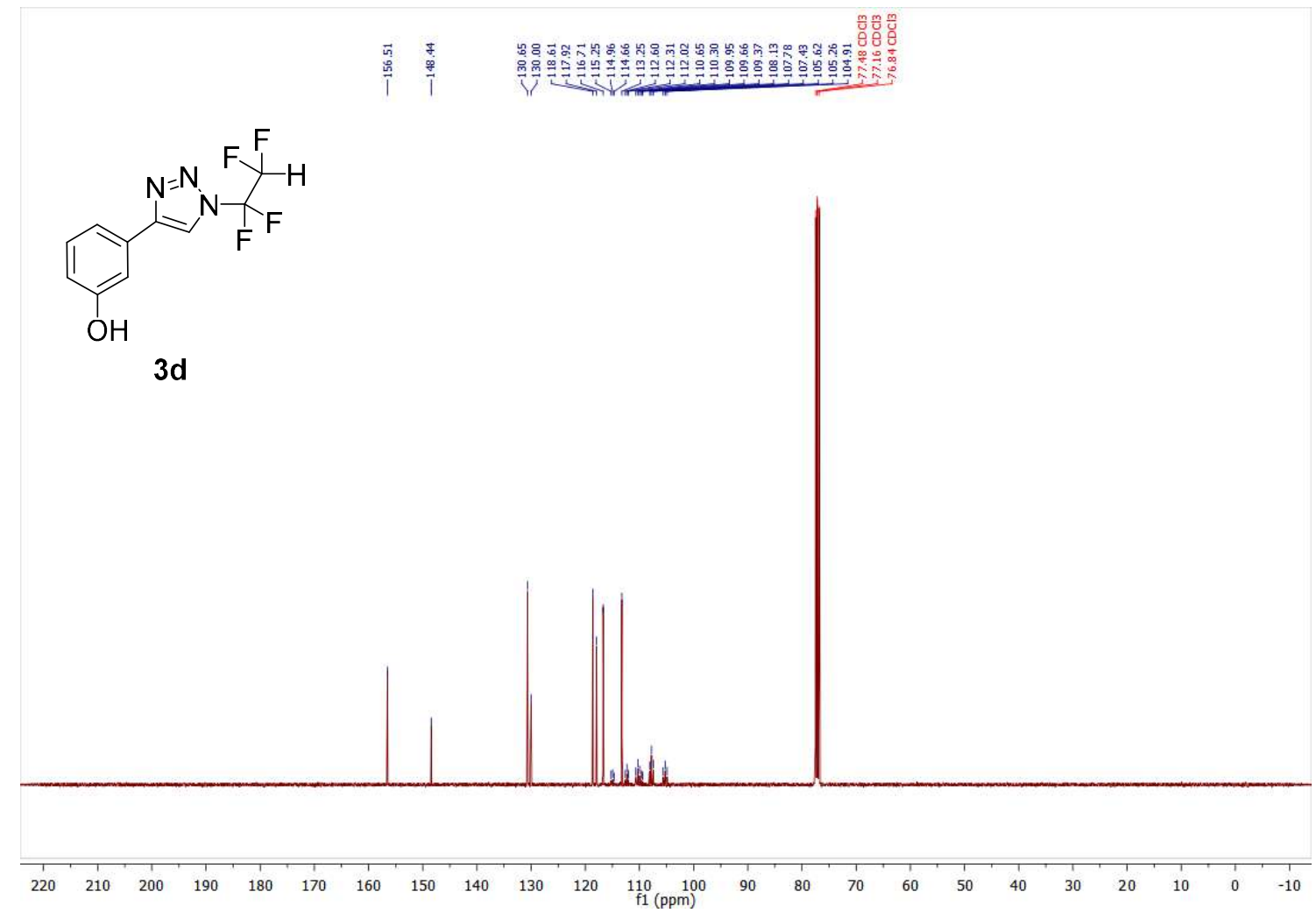

${ }^{19} \mathrm{~F}$ NMR spectrum of $\mathbf{3 d}\left(\mathrm{CDCl}_{3}, 377 \mathrm{MHz}\right)$

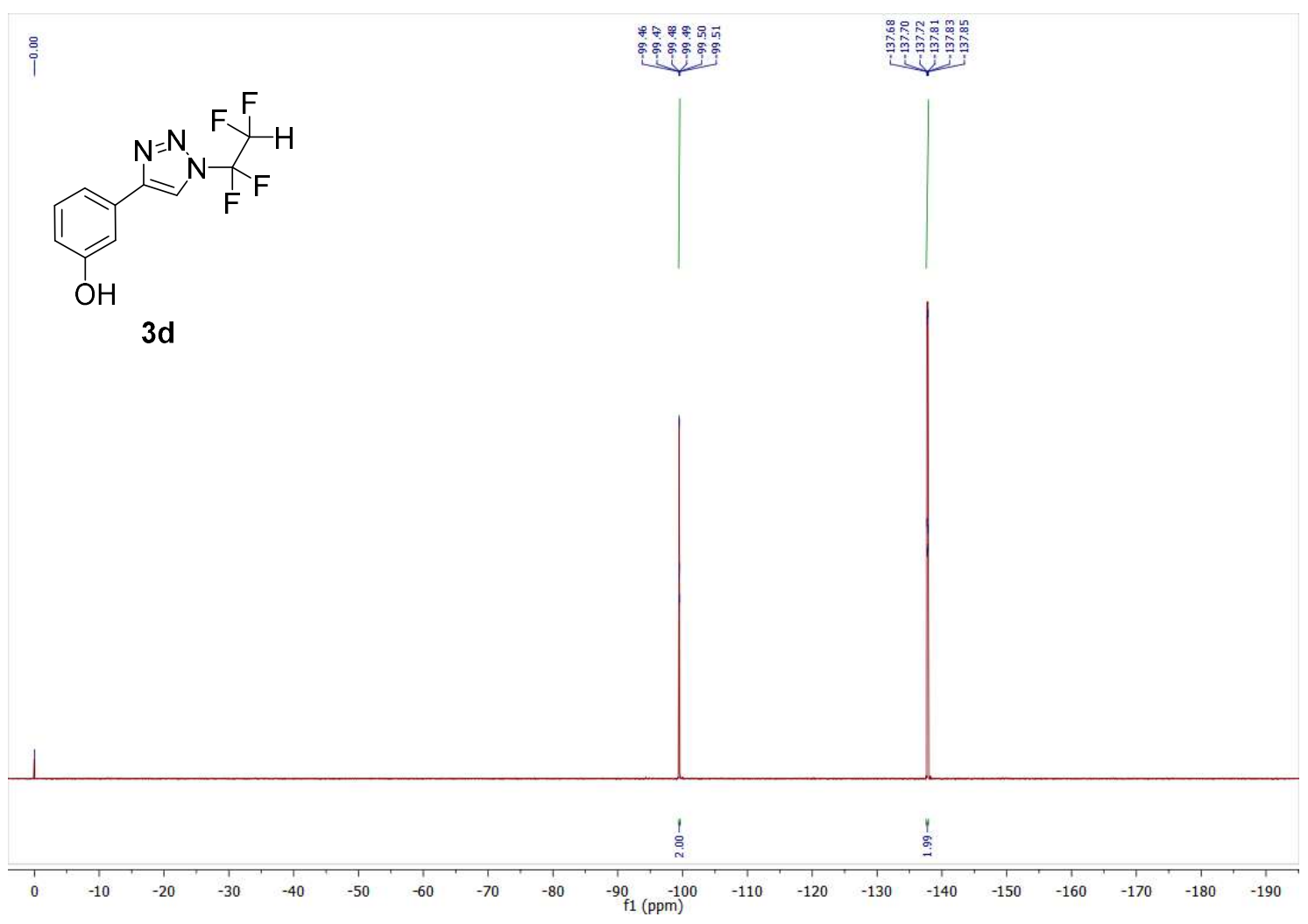


${ }^{1} \mathrm{H}$ NMR spectrum of $\mathbf{3 f}\left(\mathrm{CDCl}_{3}, 400 \mathrm{MHz}\right)$

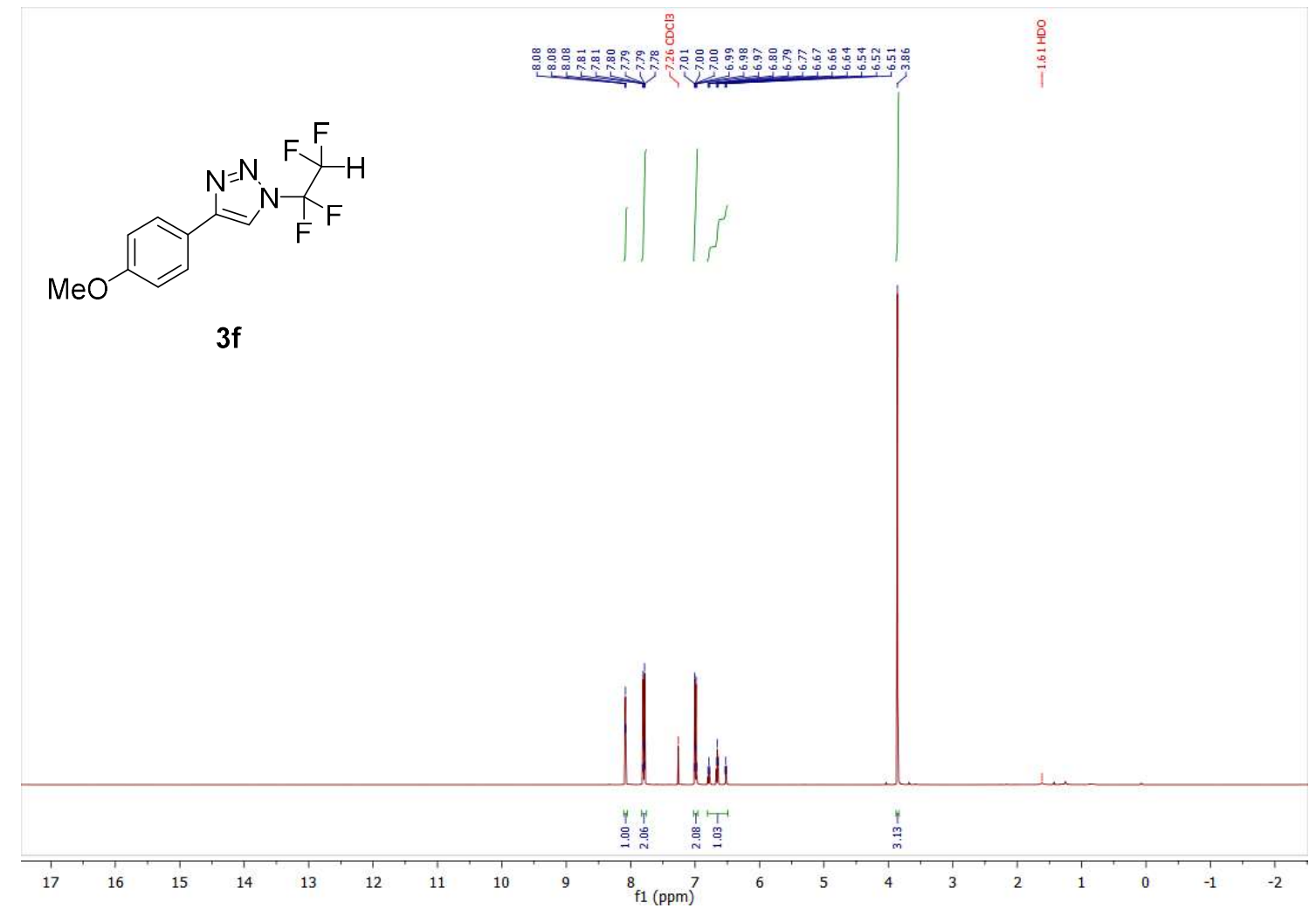

${ }^{13} \mathrm{C}\left\{{ }^{1} \mathrm{H}\right\}$ NMR spectrum of $\mathbf{3 f}\left(\mathrm{CDCl}_{3}, 101 \mathrm{MHz}\right)$

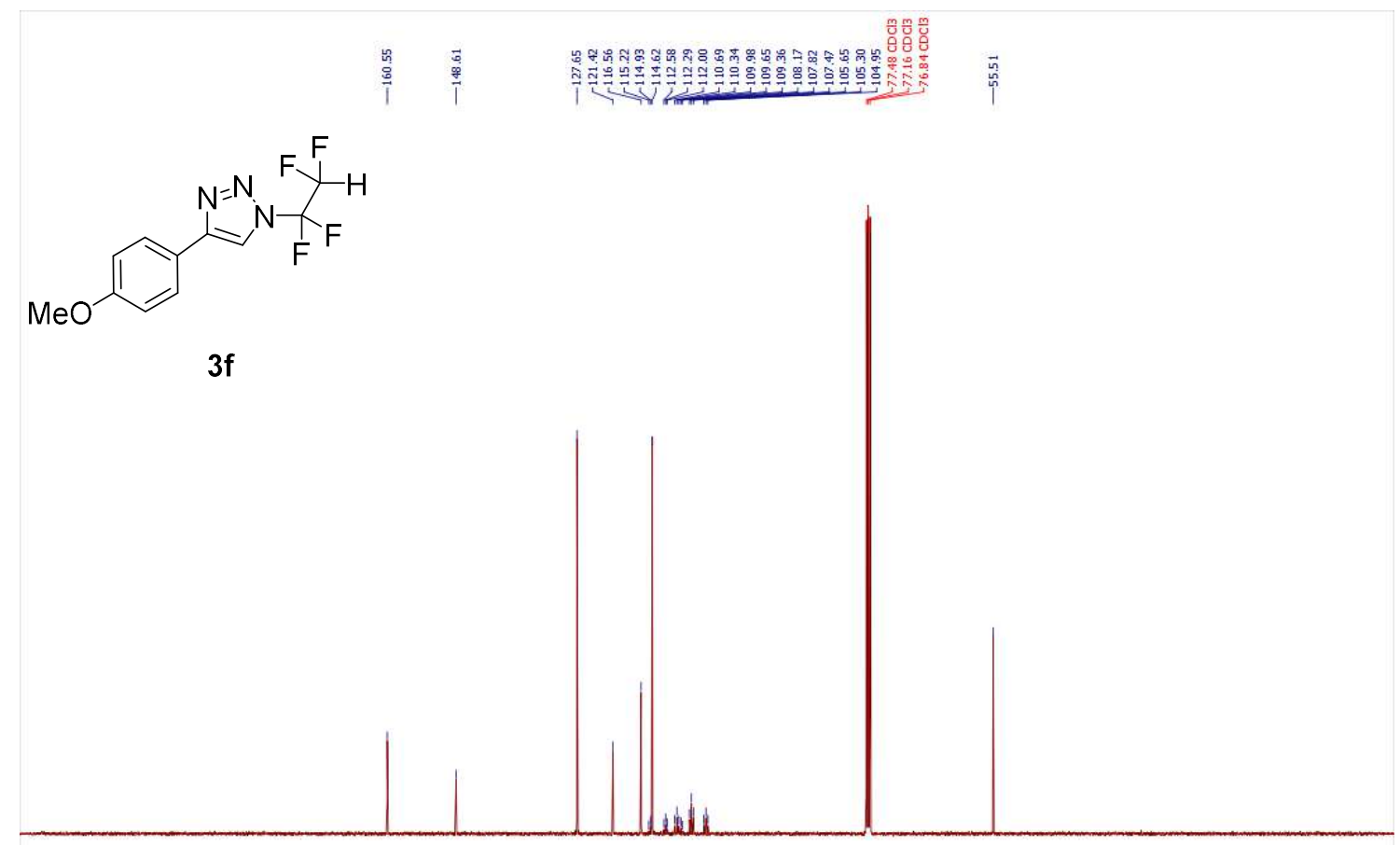

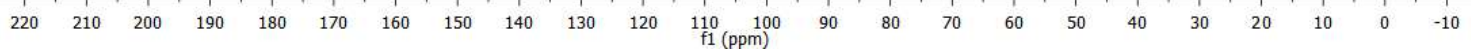


${ }^{19} \mathrm{~F} \mathrm{NMR} \mathrm{spectrum} \mathrm{of} \mathbf{3 f}\left(\mathrm{CDCl}_{3}, 377 \mathrm{MHz}\right)$

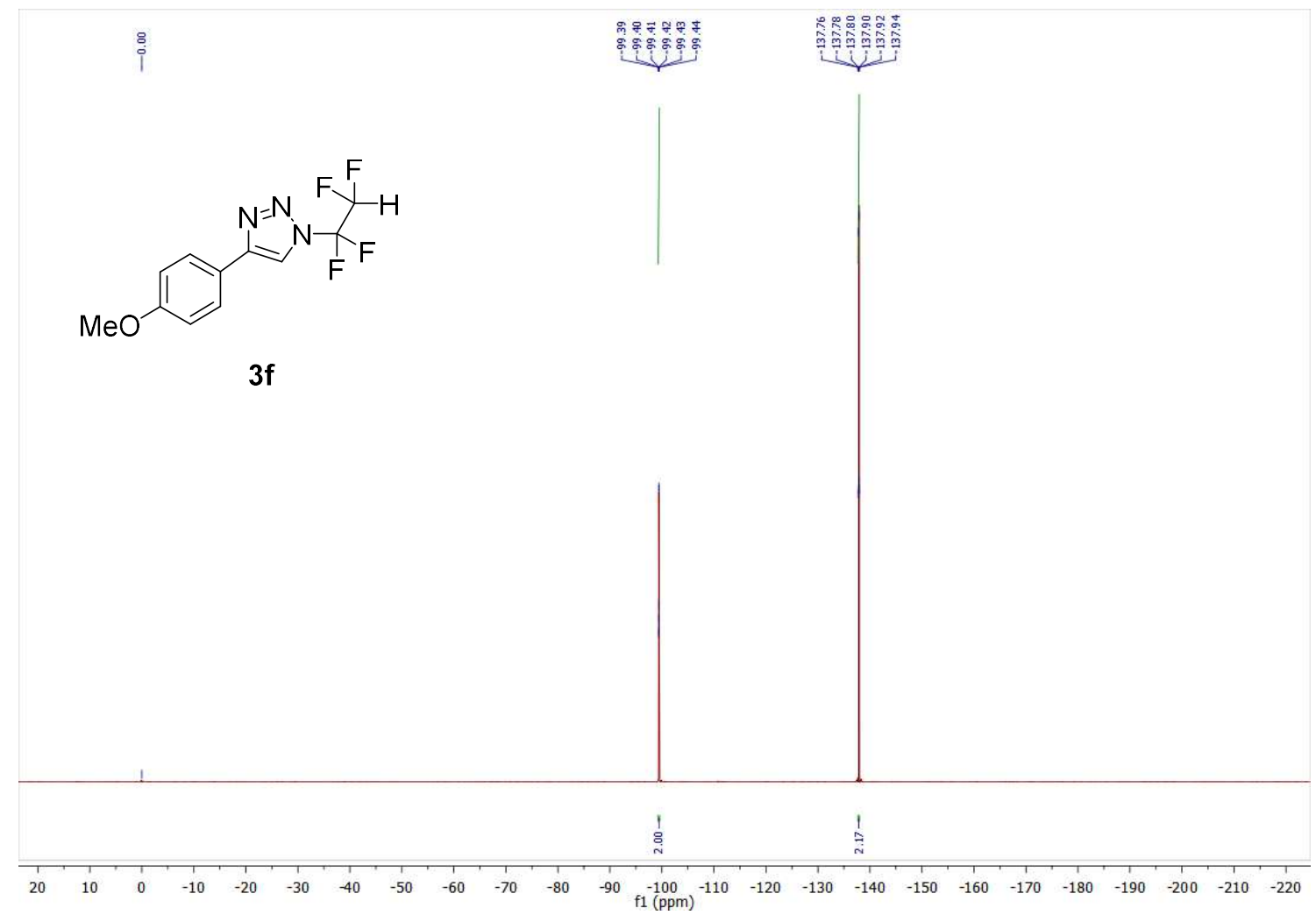

${ }^{1} \mathrm{H}$ NMR spectrum of $\mathbf{3 g}\left(\mathrm{CDCl}_{3}, 400 \mathrm{MHz}\right)$

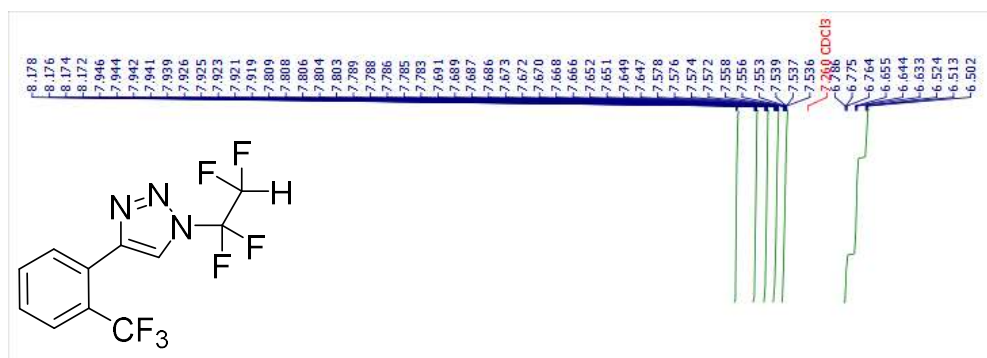

$3 \mathrm{~g}$

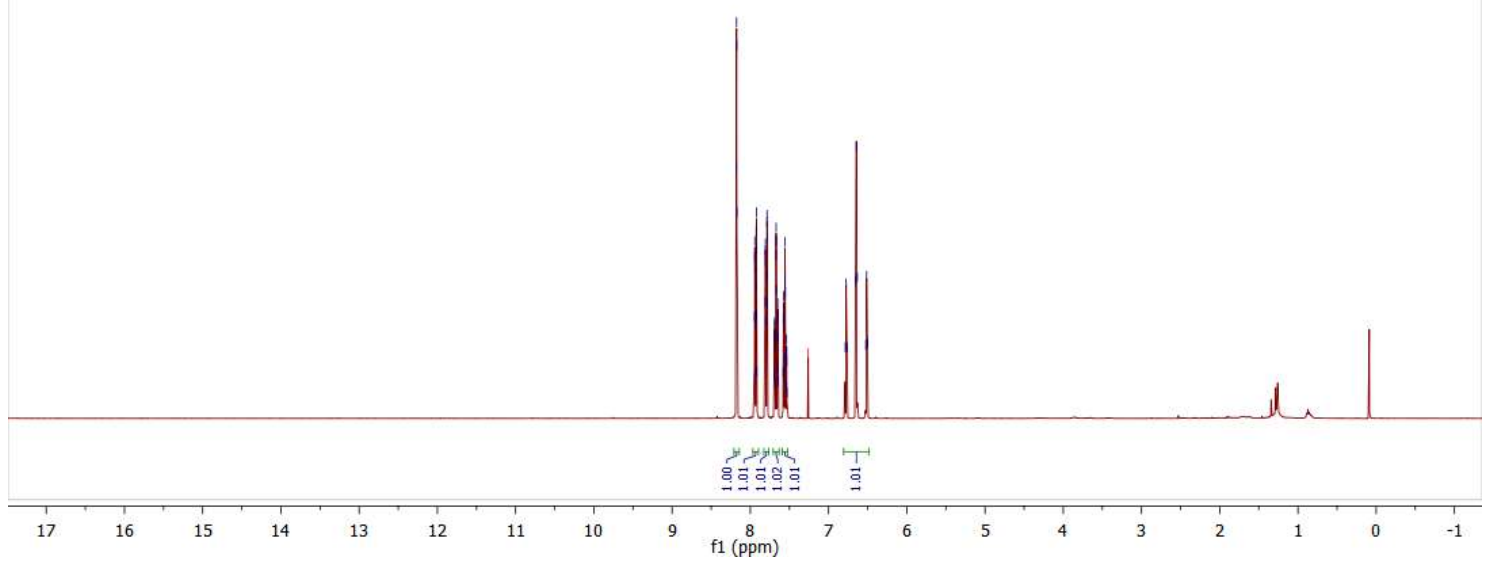


${ }^{13} \mathrm{C}\left\{{ }^{1} \mathrm{H}\right\}$ NMR spectrum of $\mathbf{3 g}\left(\mathrm{CDCl}_{3}, 101 \mathrm{MHz}\right)$
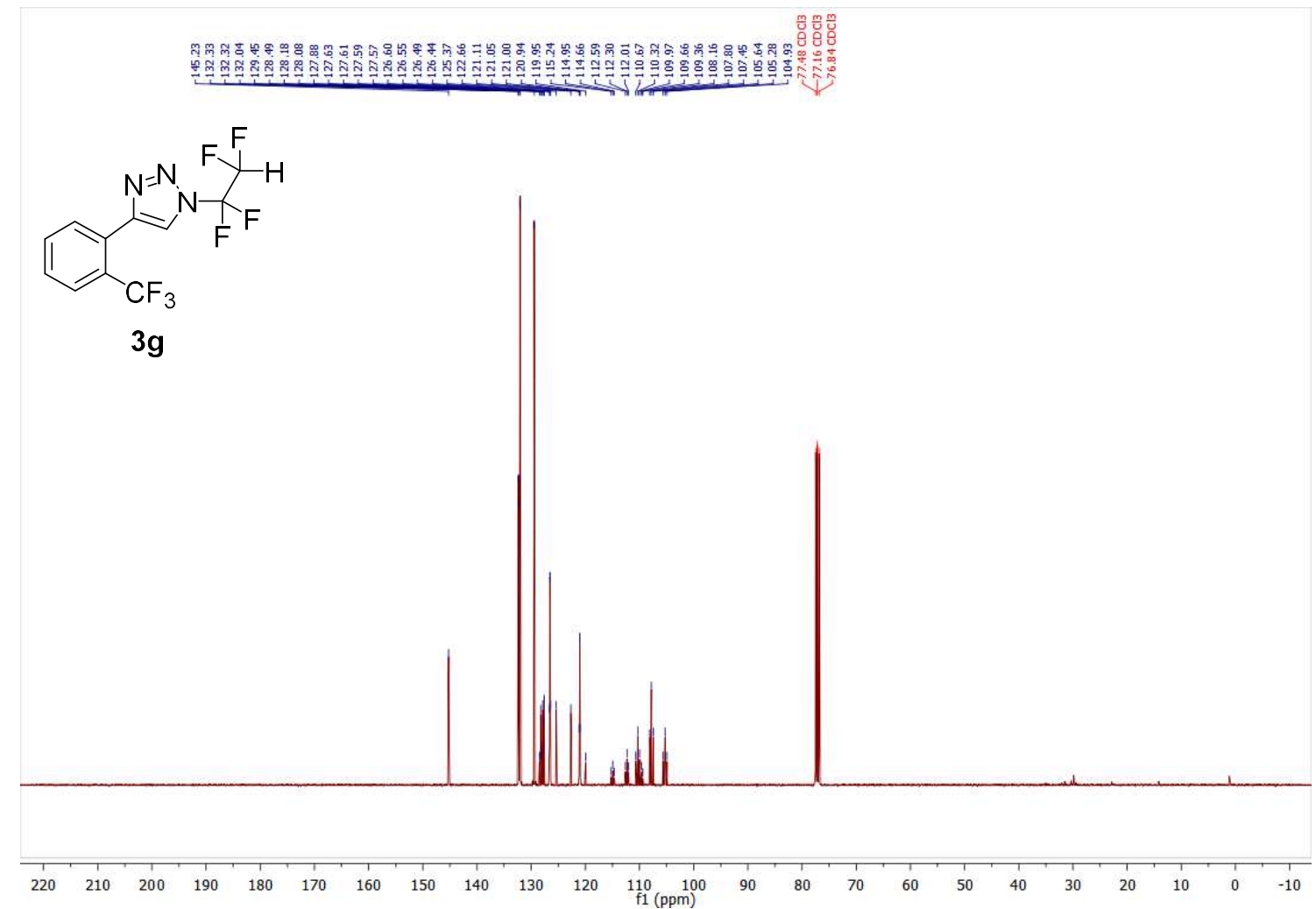

${ }^{19} \mathrm{~F}$ NMR spectrum of $\mathbf{3 g}\left(\mathrm{CDCl}_{3}, 377 \mathrm{MHz}\right)$

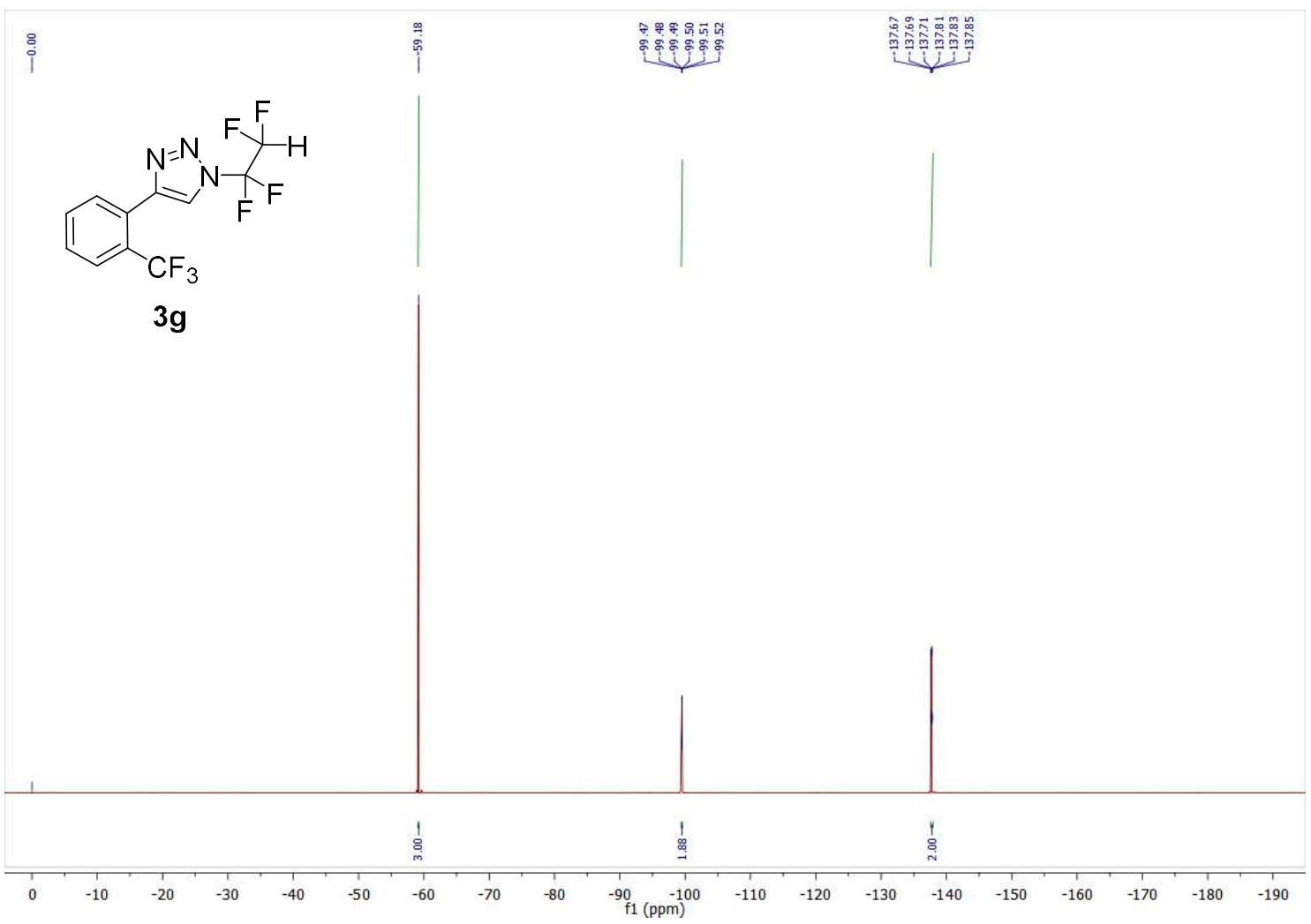


${ }^{1} \mathrm{H}$ NMR spectrum of $\mathbf{3 h}\left(\mathrm{CDCl}_{3}, 400 \mathrm{MHz}\right)$

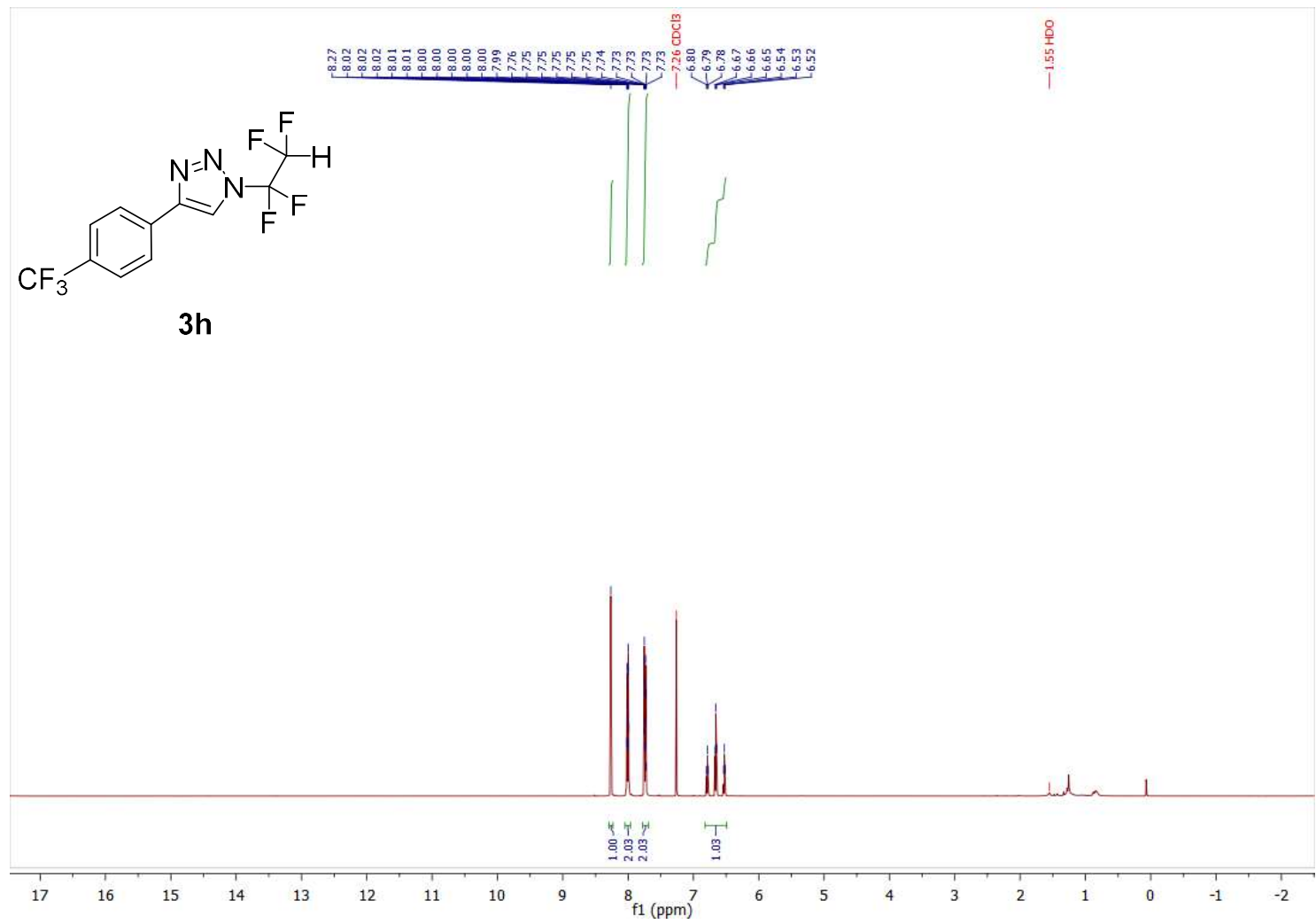

${ }^{13} \mathrm{C}\left\{{ }^{1} \mathrm{H}\right\}$ NMR spectrum of $\mathbf{3 h}\left(\mathrm{CDCl}_{3}, 101 \mathrm{MHz}\right)$

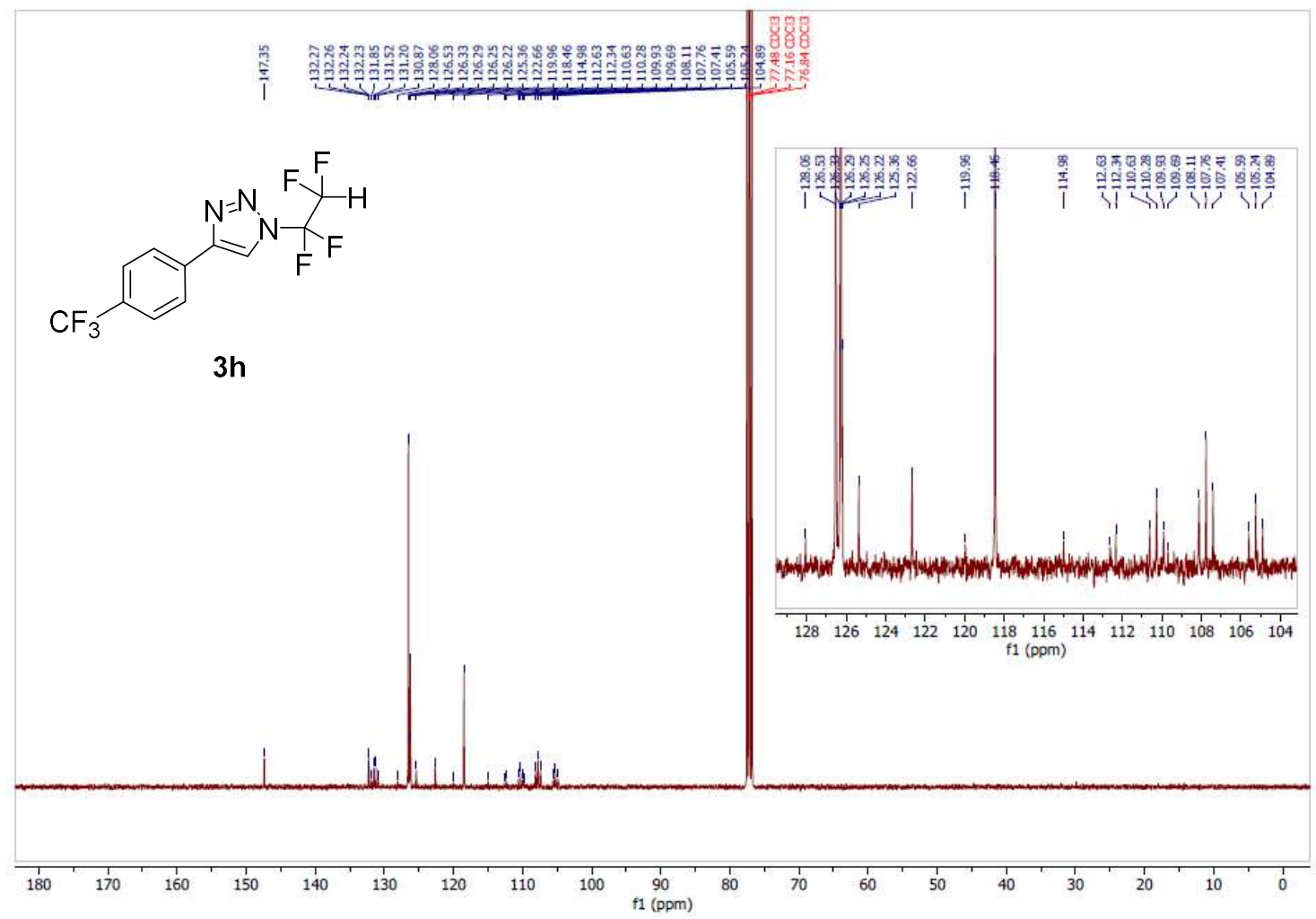


${ }^{19} \mathrm{~F} \mathrm{NMR}$ spectrum of $\mathbf{3 h}\left(\mathrm{CDCl}_{3}, 377 \mathrm{MHz}\right)$

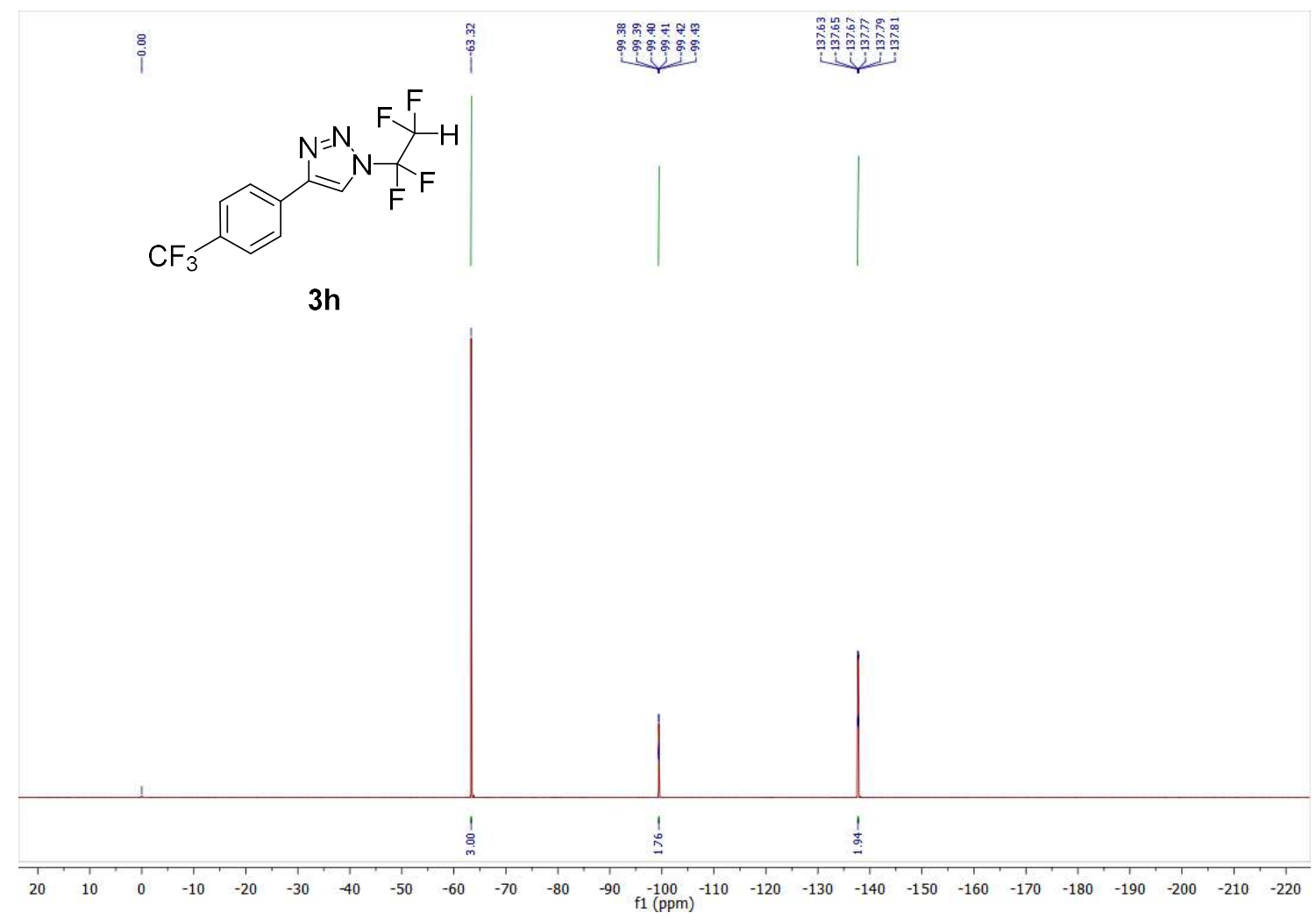

${ }^{1} \mathrm{H}$ NMR spectrum of $3 \mathbf{i}\left(\mathrm{CDCl}_{3}, 400 \mathrm{MHz}\right)$ 


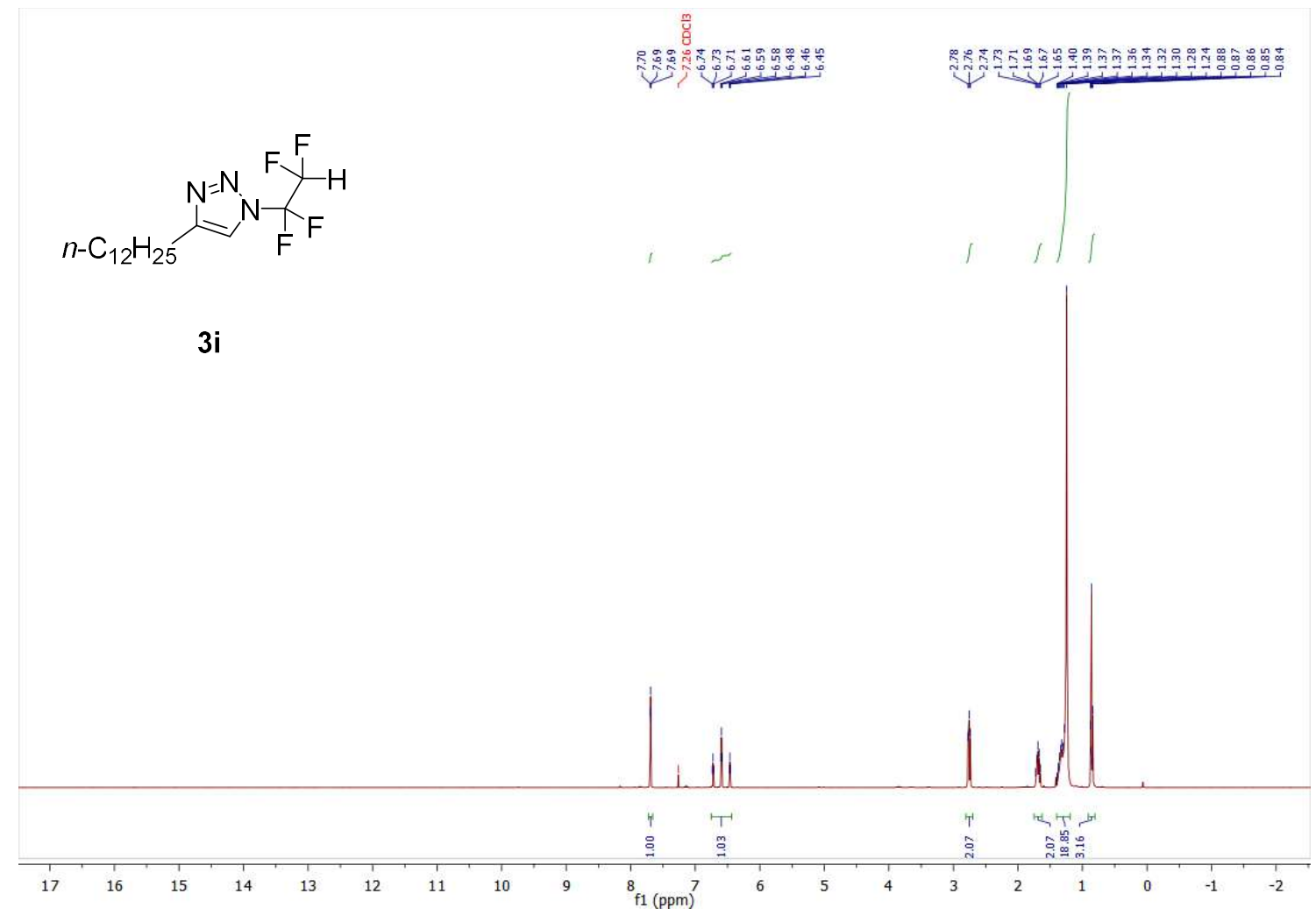

${ }^{13} \mathrm{C}\left\{{ }^{1} \mathrm{H}\right\}$ NMR spectrum of $\mathbf{3 i}\left(\mathrm{CDCl}_{3}, 101 \mathrm{MHz}\right)$
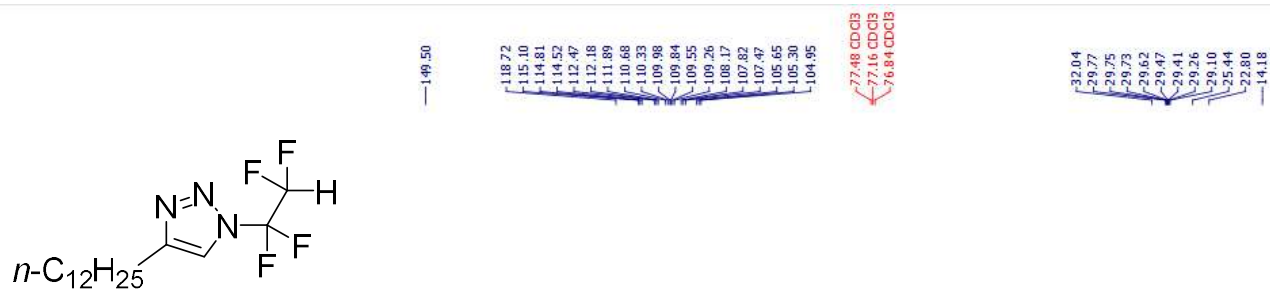

$3 \mathbf{i}$
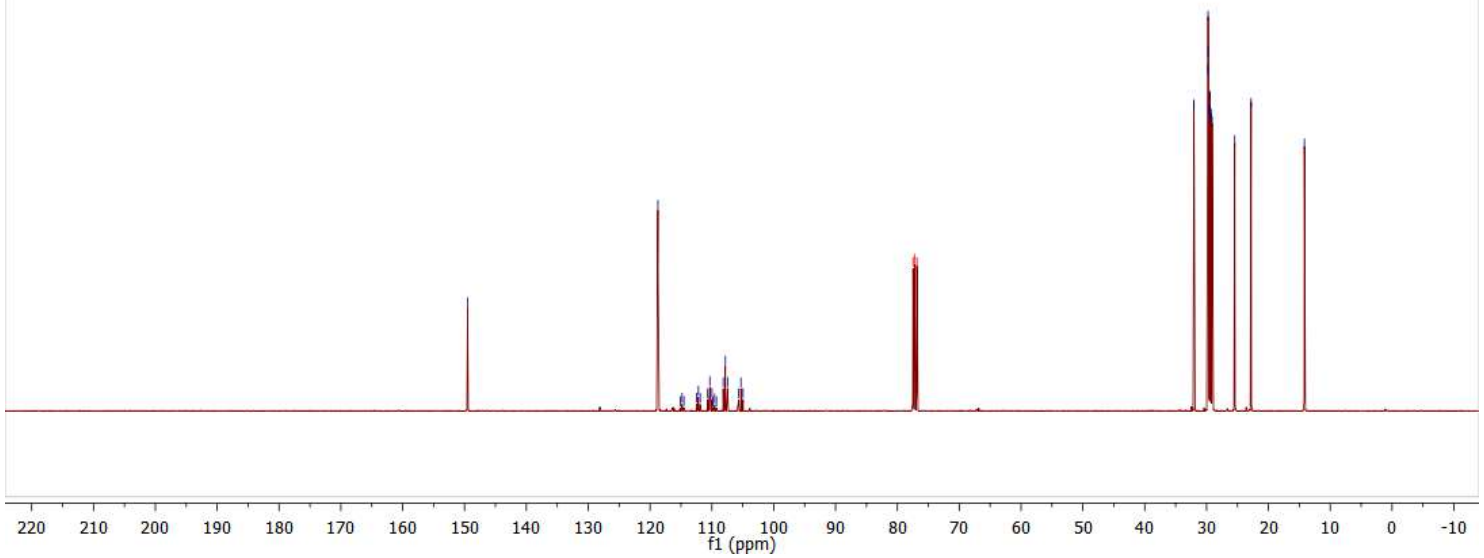

${ }^{19} \mathrm{~F}$ NMR spectrum of $\mathbf{3 i}\left(\mathrm{CDCl}_{3}, 377 \mathrm{MHz}\right)$ 


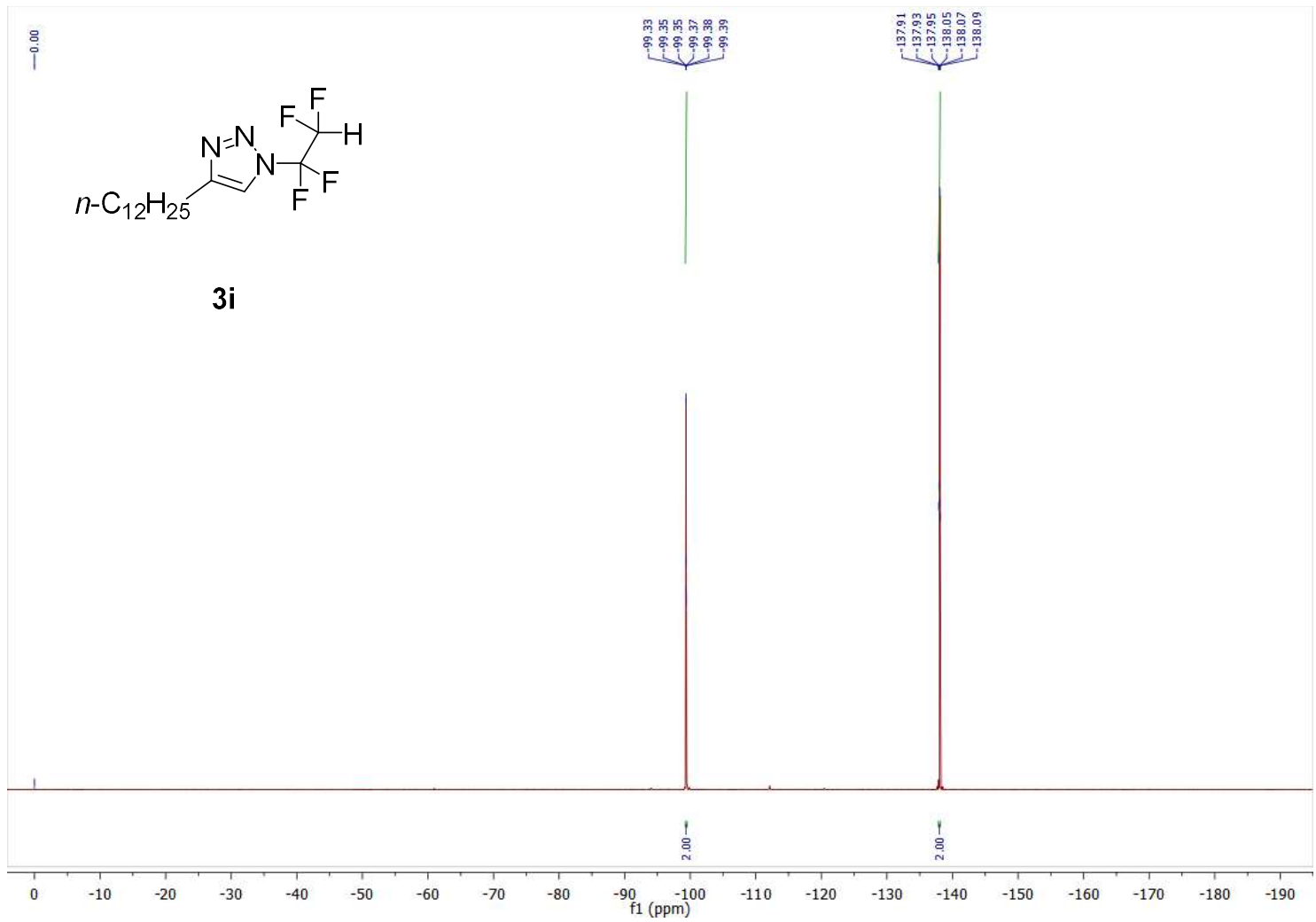

${ }^{1} \mathrm{H}$ NMR spectrum of $4\left(\mathrm{CDCl}_{3}, 400 \mathrm{MHz}\right)$

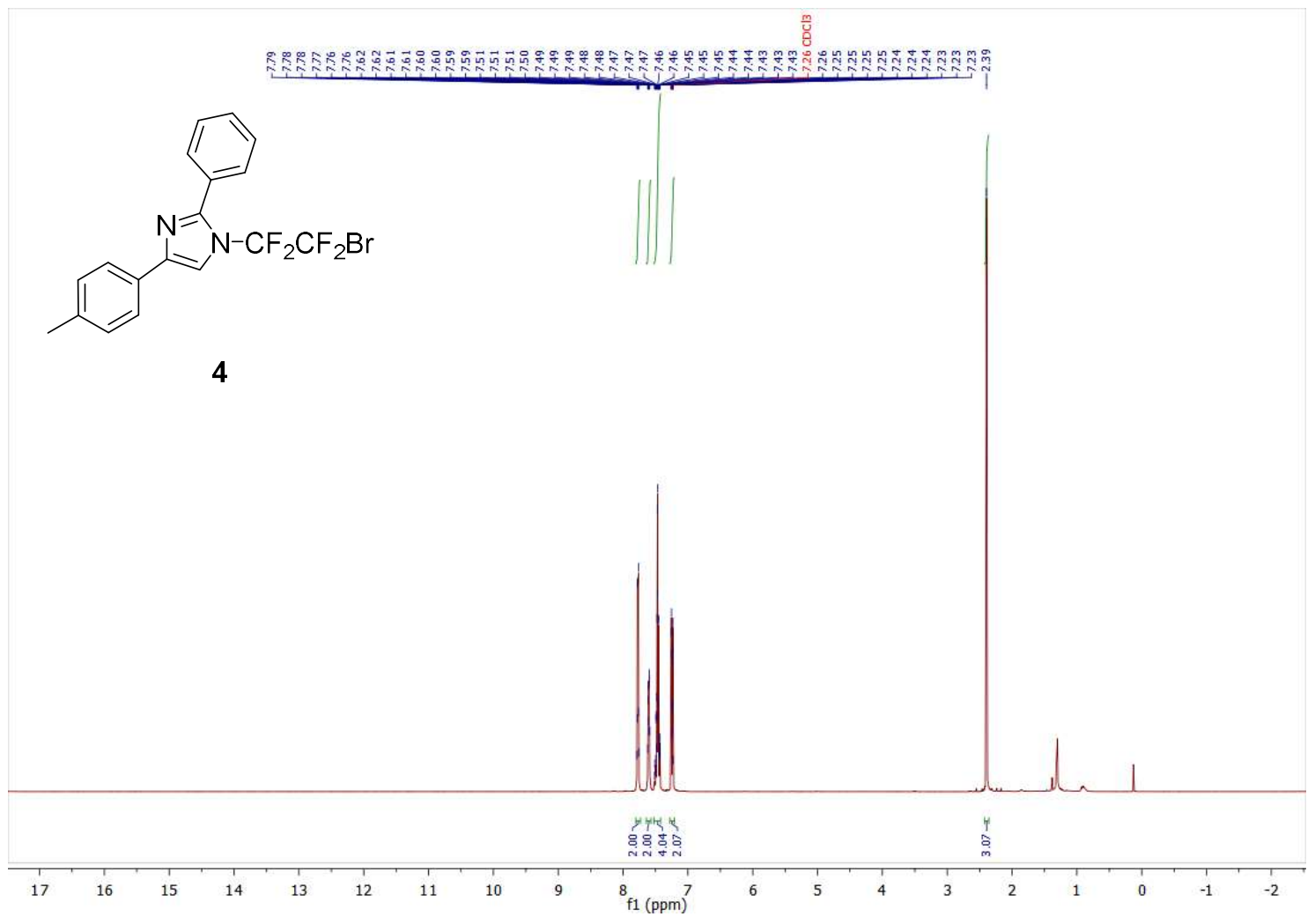

${ }^{13} \mathrm{C}\left\{{ }^{1} \mathrm{H}\right\}$ NMR spectrum of $4\left(\mathrm{CDCl}_{3}, 101 \mathrm{MHz}\right)$ 

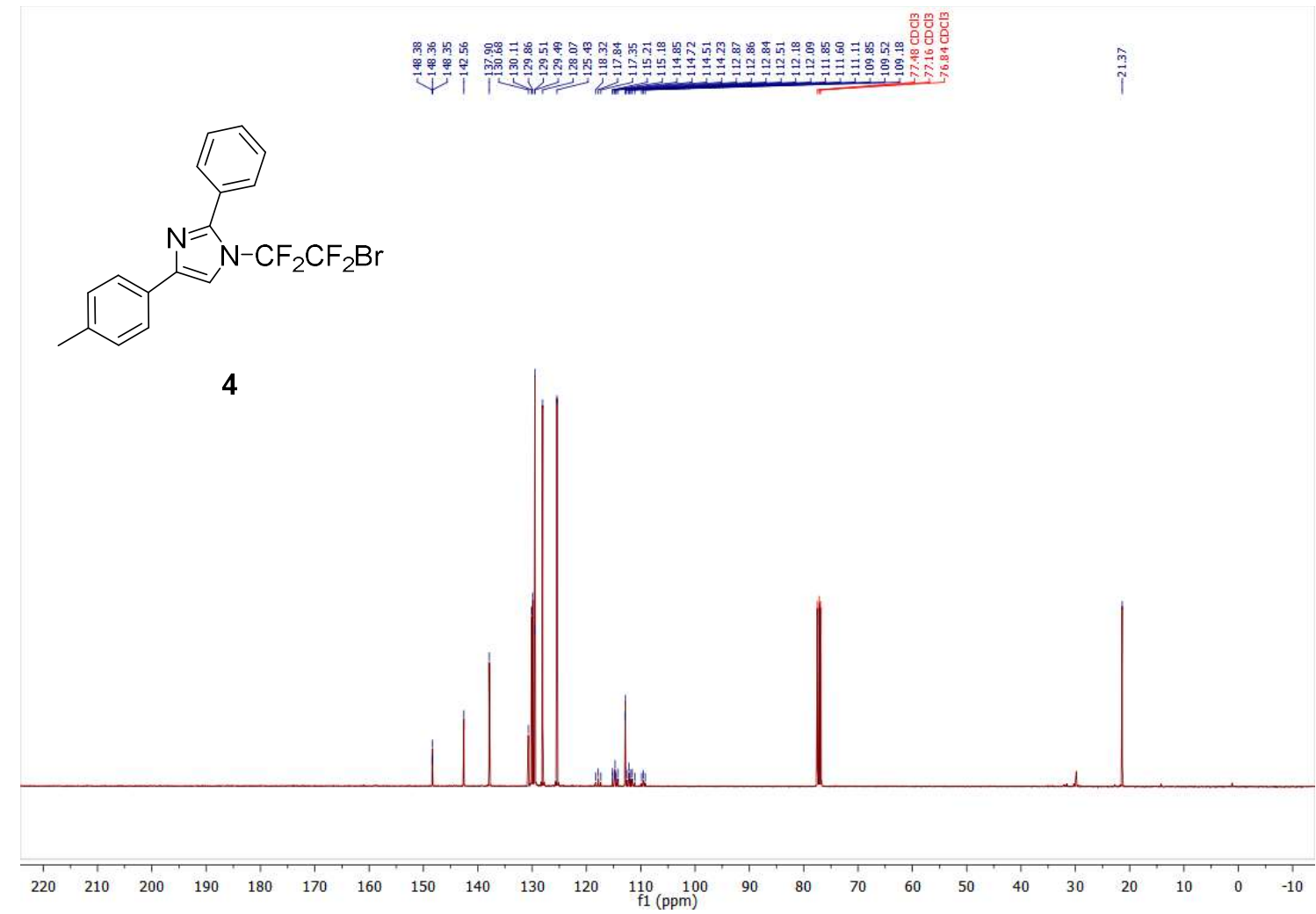

${ }^{19} \mathrm{~F}$ NMR spectrum of $4\left(\mathrm{CDCl}_{3}, 377 \mathrm{MHz}\right)$

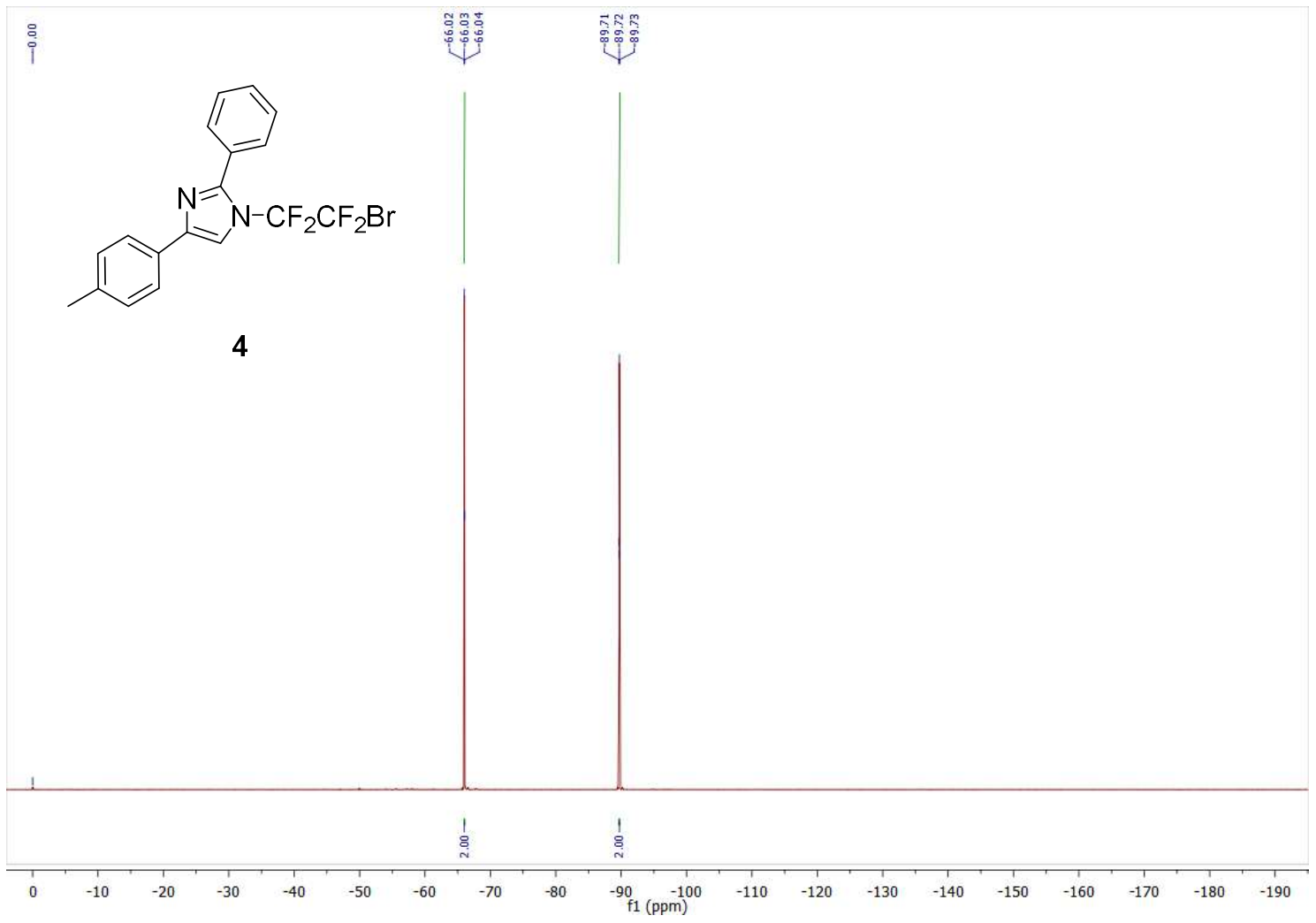

${ }^{1} \mathrm{H}$ NMR spectrum of $5\left(\mathrm{CDCl}_{3}, 400 \mathrm{MHz}\right)$ 


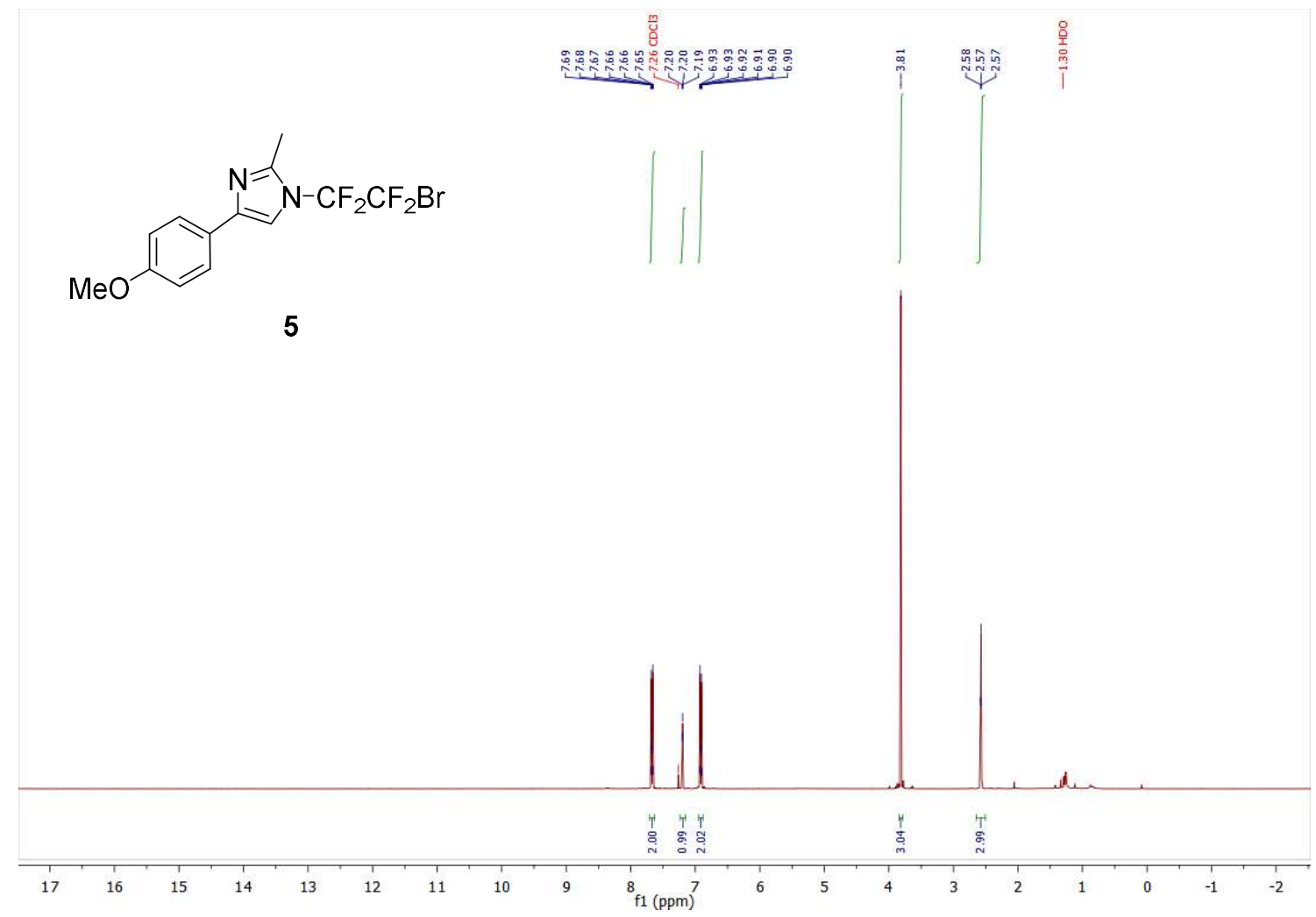

${ }^{13} \mathrm{C}\left\{{ }^{1} \mathrm{H}\right\}$ NMR spectrum of $5\left(\mathrm{CDCl}_{3}, 101 \mathrm{MHz}\right)$
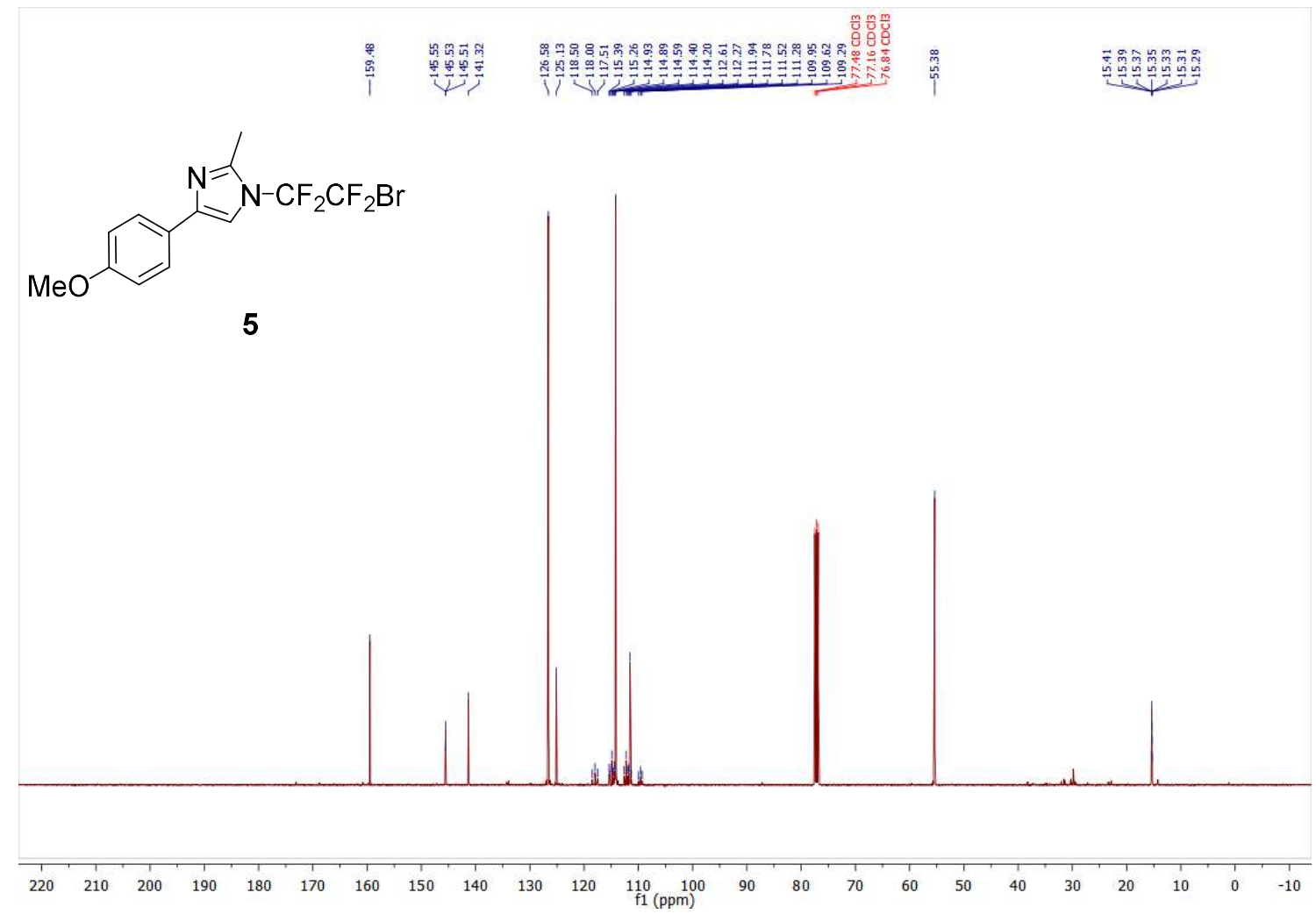

${ }^{19} \mathrm{~F}$ NMR spectrum of $5\left(\mathrm{CDCl}_{3}, 377 \mathrm{MHz}\right)$ 


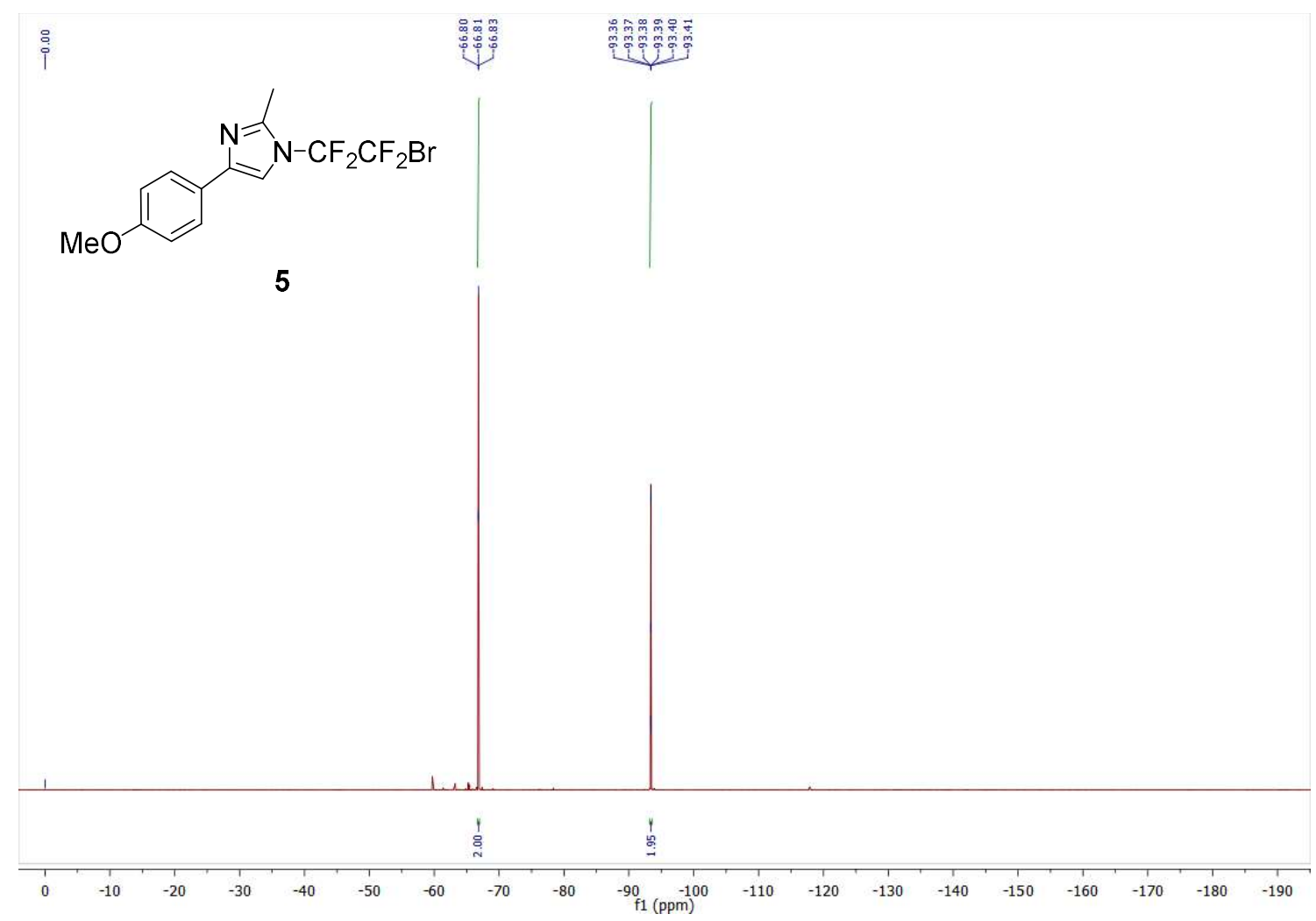

${ }^{1} \mathrm{H}$ NMR spectrum of $6\left(\mathrm{CDCl}_{3}, 400 \mathrm{MHz}\right)$

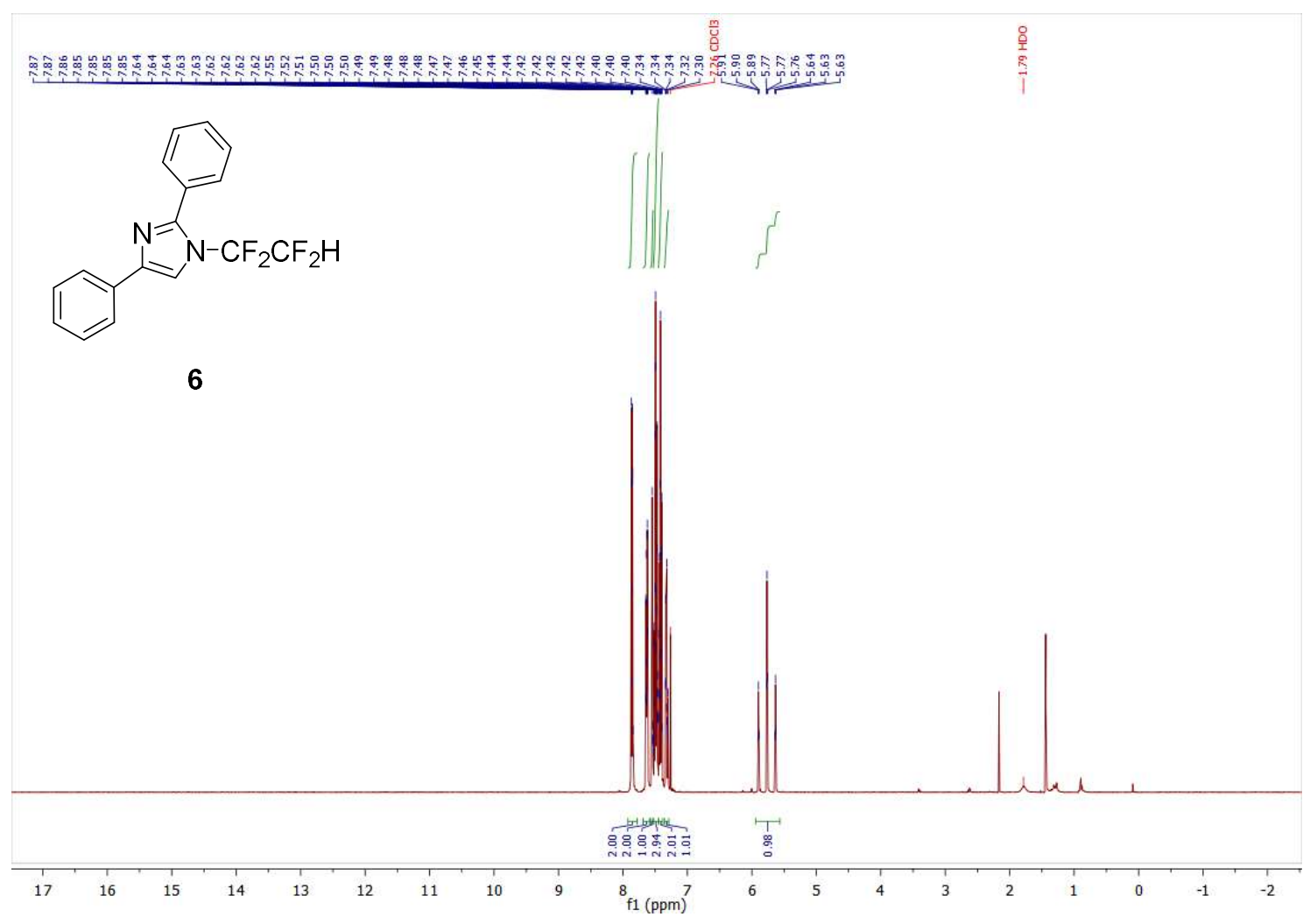

${ }^{13} \mathrm{C}\left\{{ }^{1} \mathrm{H}\right\}$ NMR spectrum of $6\left(\mathrm{CDCl}_{3}, 101 \mathrm{MHz}\right)$ 

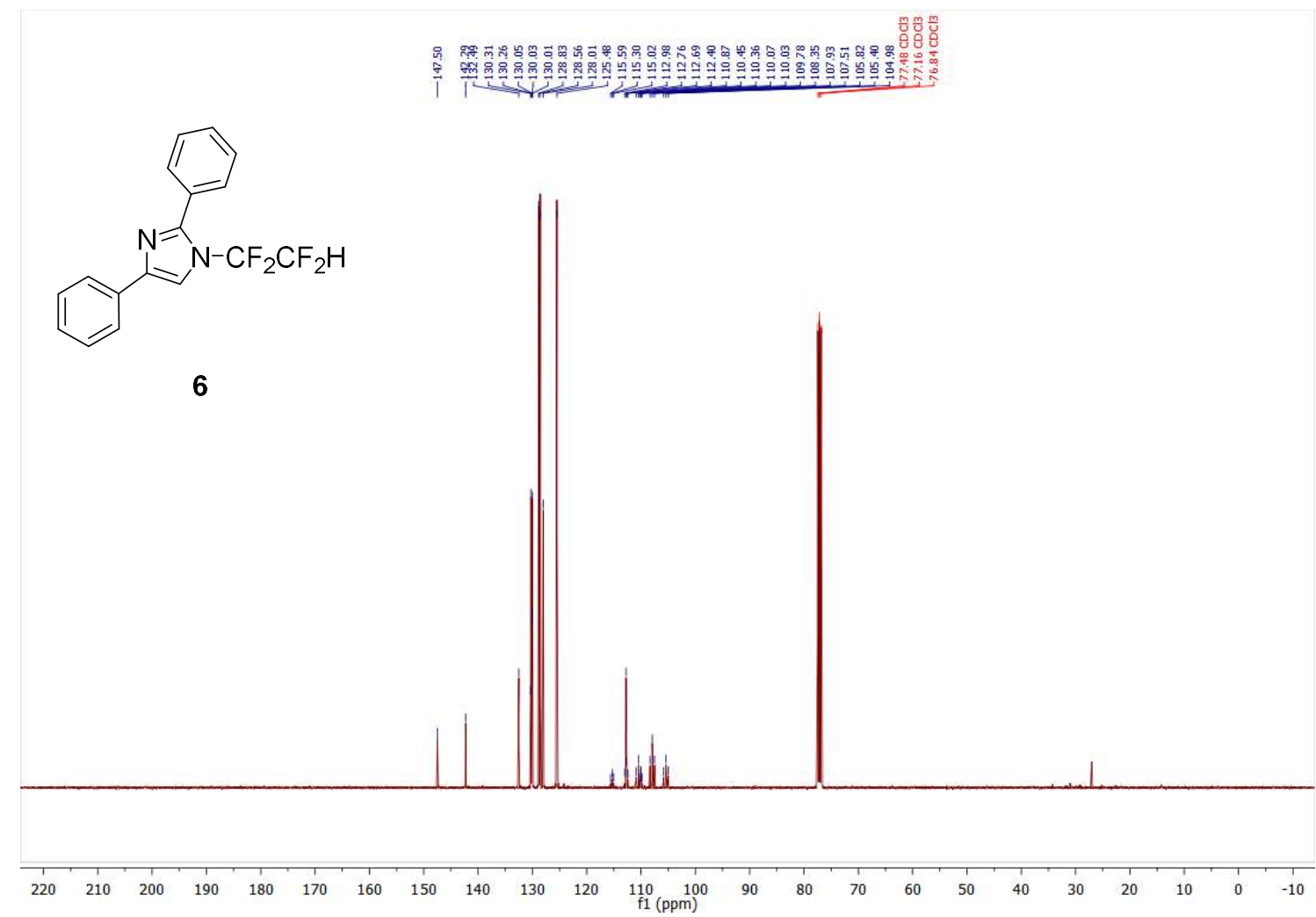

${ }^{19} \mathrm{~F} \mathrm{NMR} \mathrm{spectrum} \mathrm{of} 6\left(\mathrm{CDCl}_{3}, 377 \mathrm{MHz}\right)$

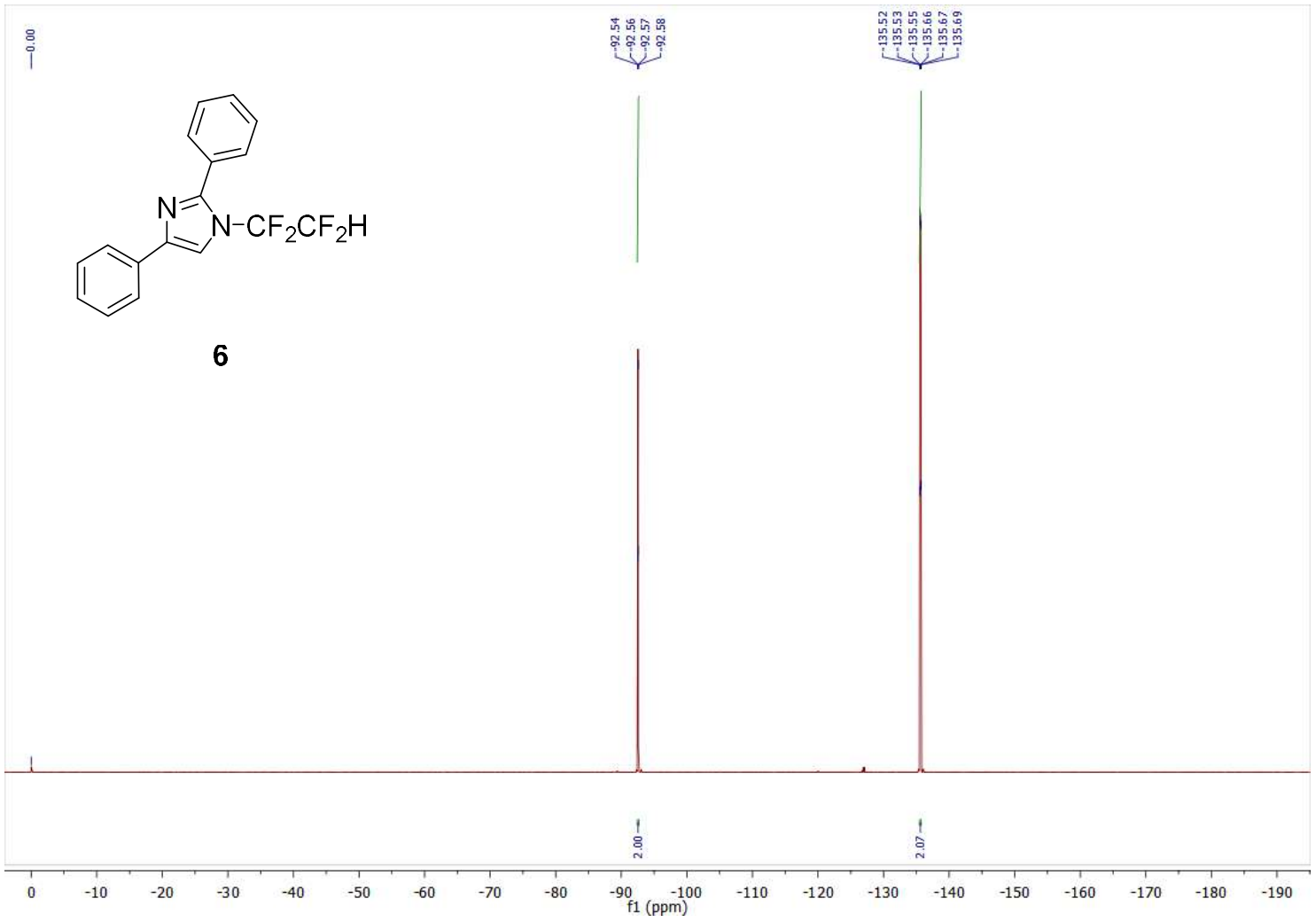

${ }^{1} \mathrm{H}$ NMR spectrum of $7\left(\mathrm{CDCl}_{3}, 400 \mathrm{MHz}\right)$ 


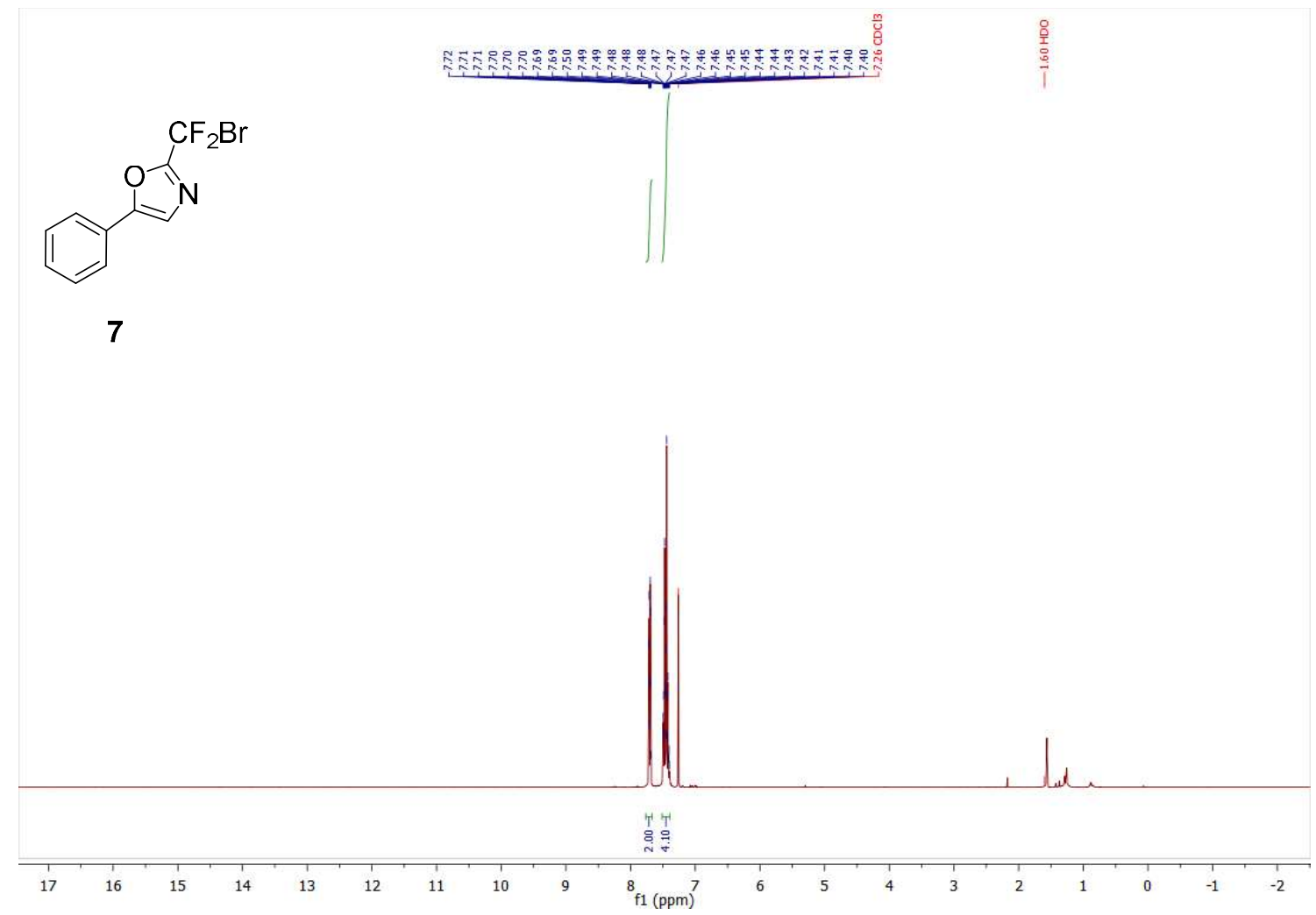

${ }^{13} \mathrm{C}\left\{{ }^{1} \mathrm{H}\right\}$ NMR spectrum of $\mathbf{7}\left(\mathrm{CDCl}_{3}, 101 \mathrm{MHz}\right)$

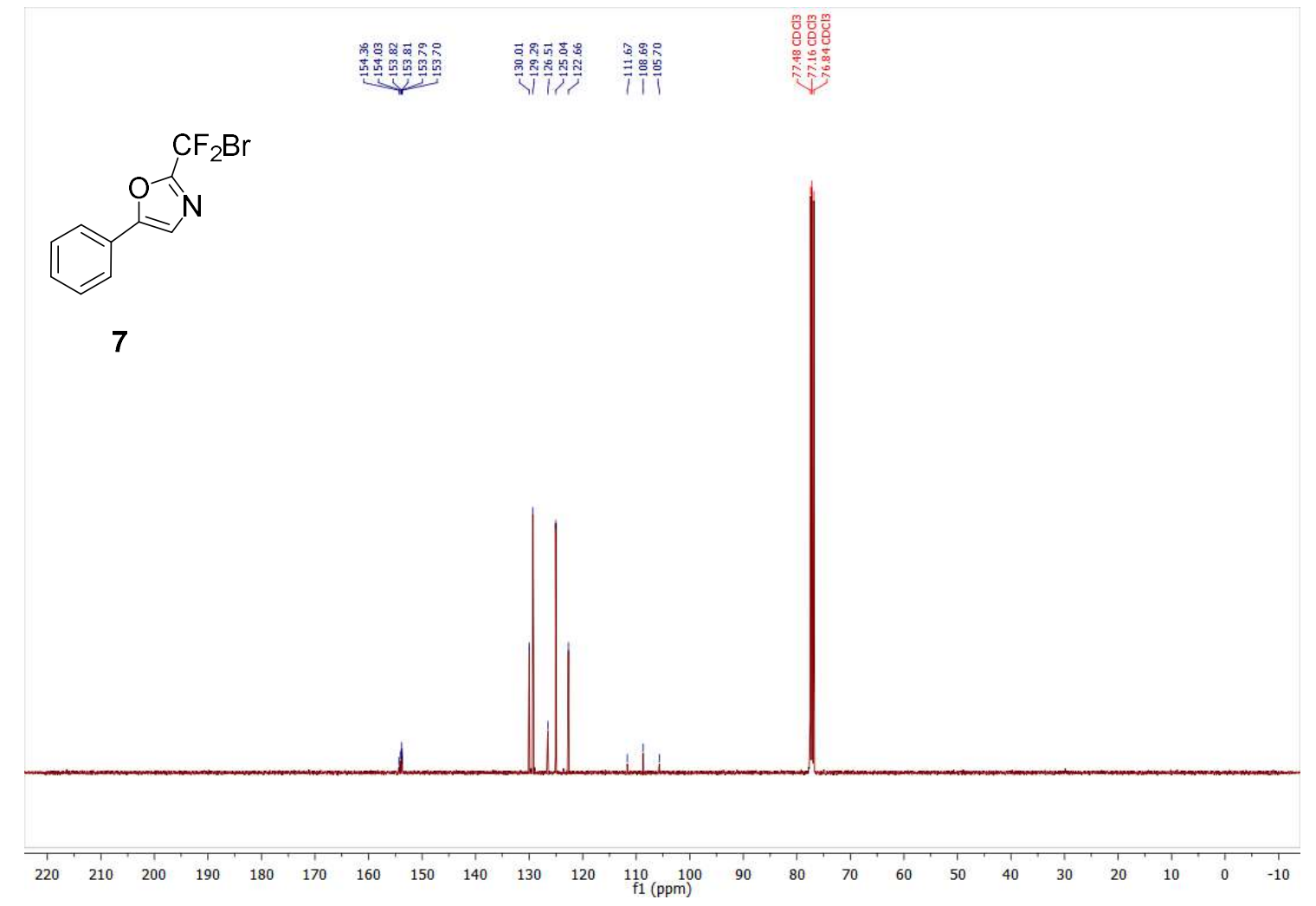

${ }^{19} \mathrm{~F}$ NMR spectrum of $7\left(\mathrm{CDCl}_{3}, 377 \mathrm{MHz}\right)$ 


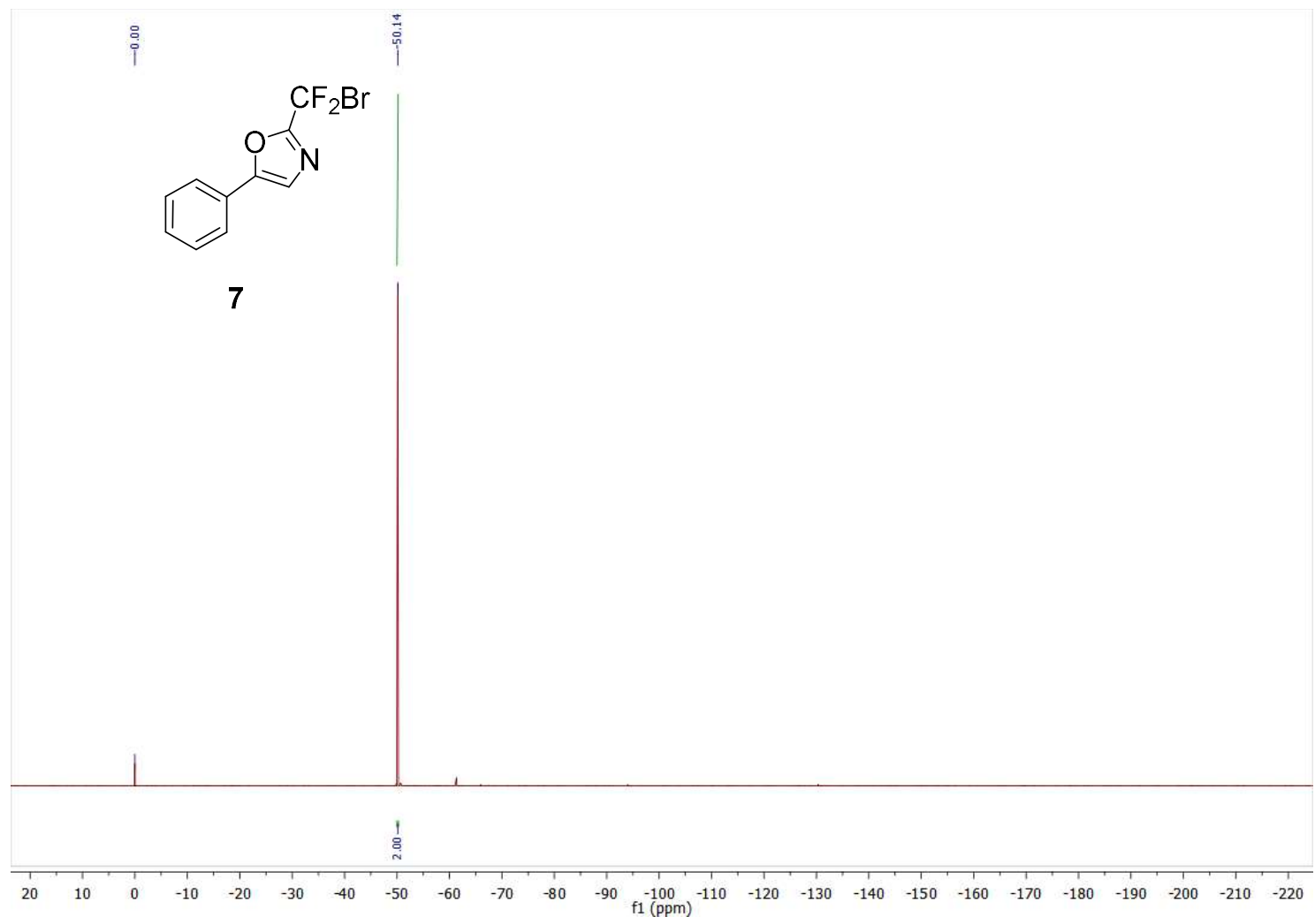

${ }^{1} \mathrm{H}$ NMR spectrum of $8\left(\mathrm{CDCl}_{3}, 400 \mathrm{MHz}\right)$

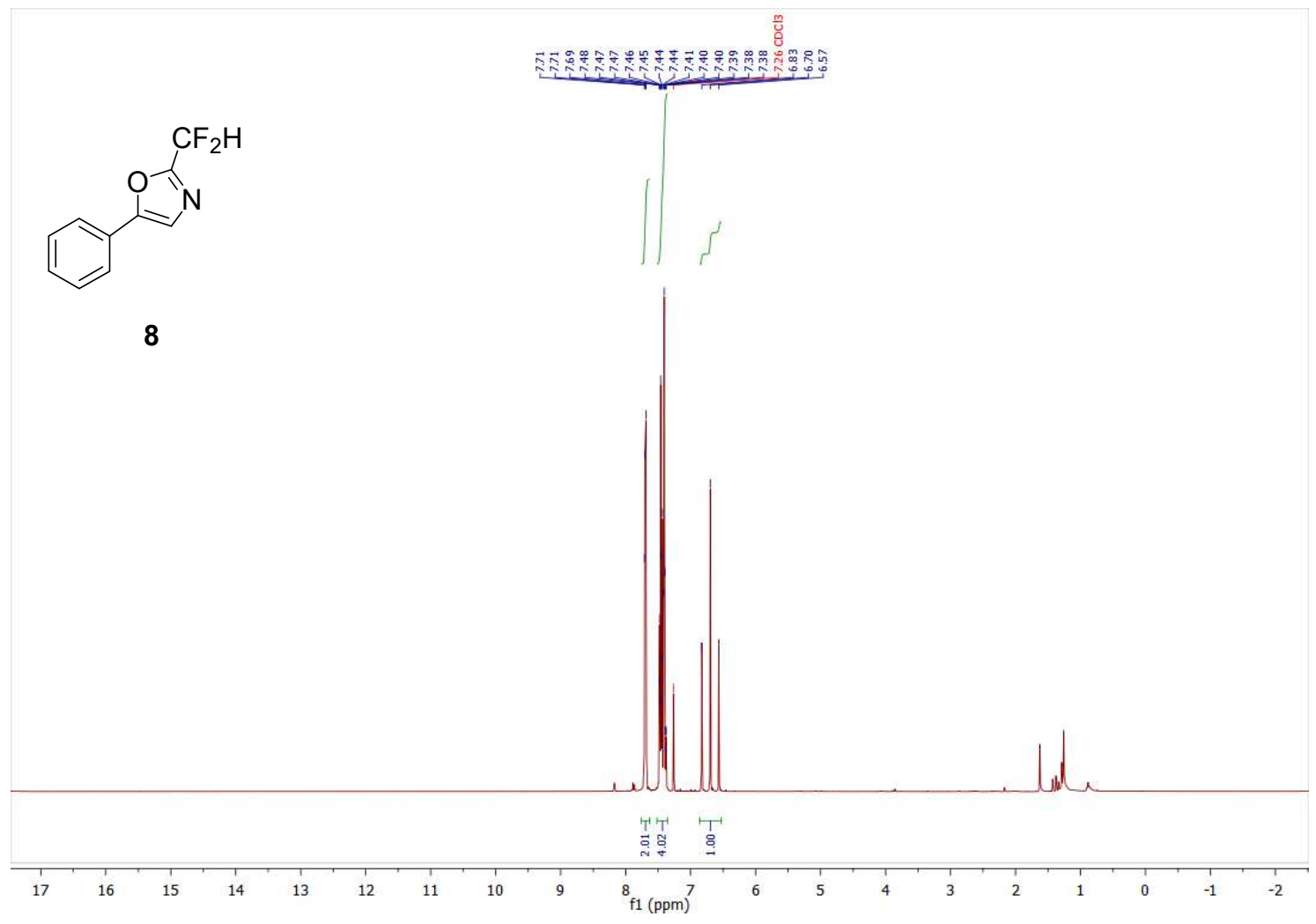

${ }^{13} \mathrm{C}\left\{{ }^{1} \mathrm{H}\right\}$ NMR spectrum of $8\left(\mathrm{CDCl}_{3}, 101 \mathrm{MHz}\right)$ 


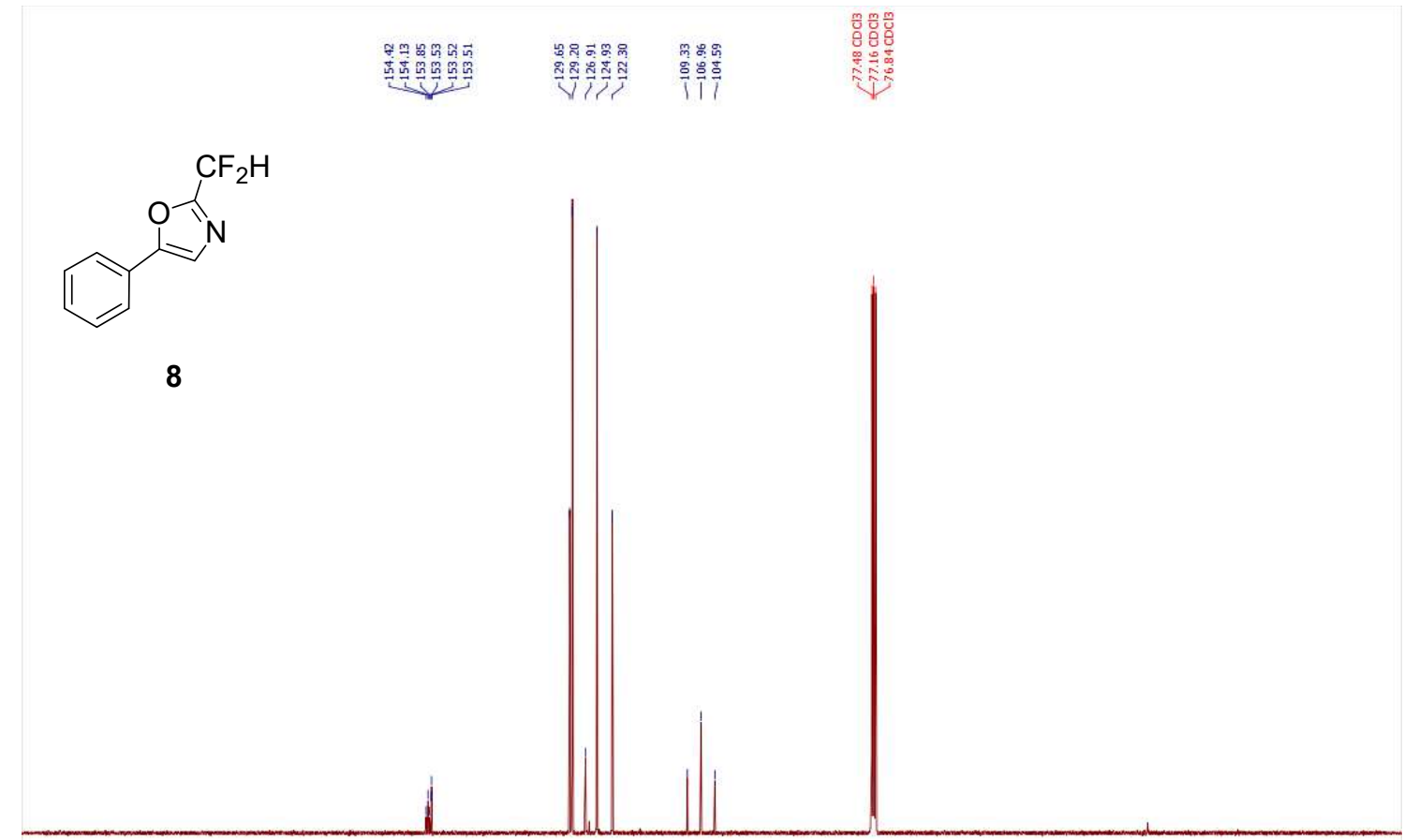

$\begin{array}{lllllllllllllllllllllllll}220 & 210 & 200 & 190 & 180 & 170 & 160 & 150 & 140 & 130 & 120 & \underset{\mathrm{f} 1}{11(\mathrm{ppm})} \mathbf{1 0 0} & 90 & 80 & 70 & 60 & 50 & 40 & 30 & 20 & 10 & 0 & -10\end{array}$

${ }^{19} \mathrm{~F} \mathrm{NMR} \mathrm{spectrum} \mathrm{of} 8\left(\mathrm{CDCl}_{3}, 377 \mathrm{MHz}\right)$

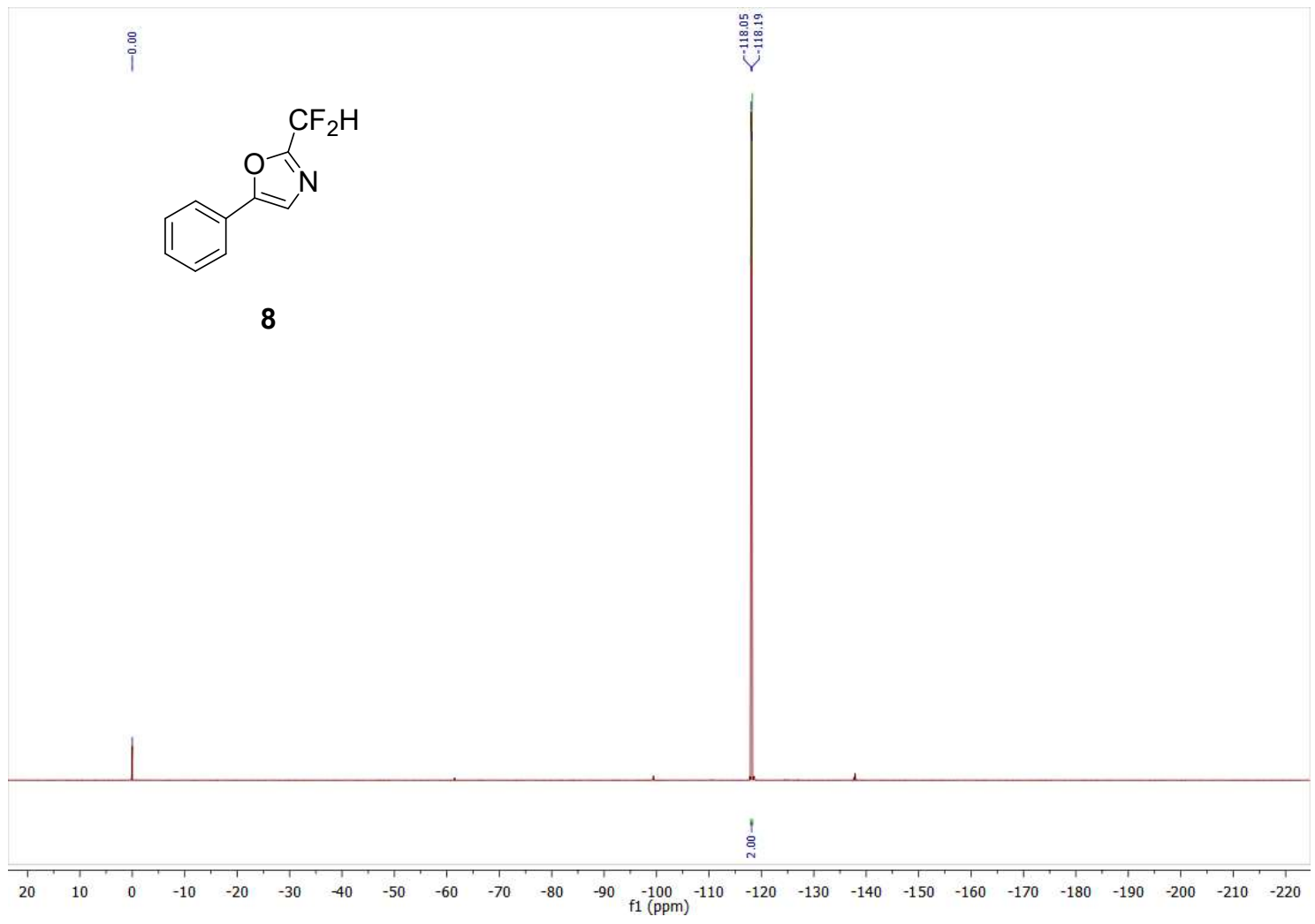

${ }^{1} \mathrm{H}$ NMR spectrum of $9\left(\mathrm{CDCl}_{3}, 400 \mathrm{MHz}\right)$ 


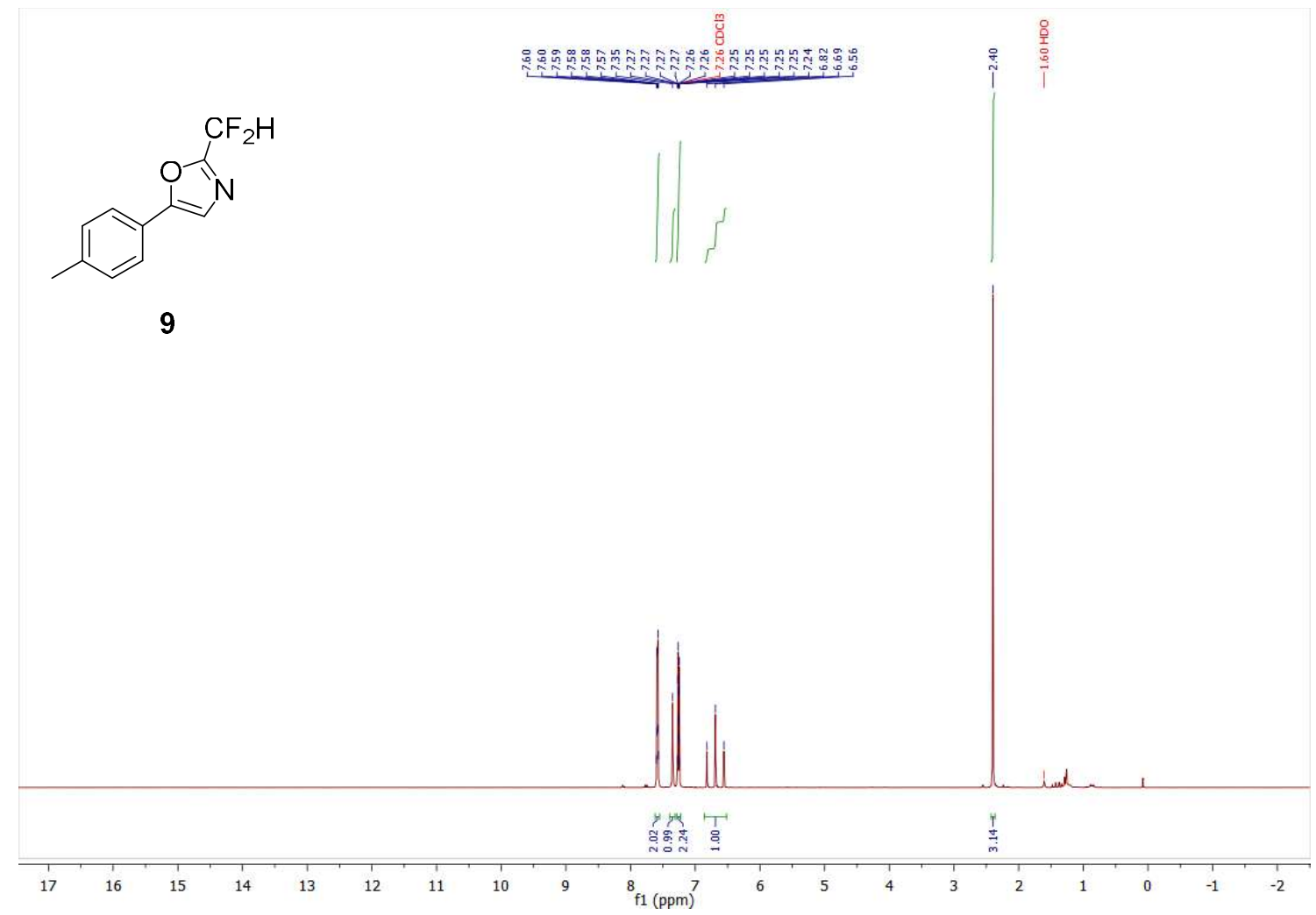

${ }^{13} \mathrm{C}\left\{{ }^{1} \mathrm{H}\right\}$ NMR spectrum of $\mathbf{9}\left(\mathrm{CDCl}_{3}, 101 \mathrm{MHz}\right)$

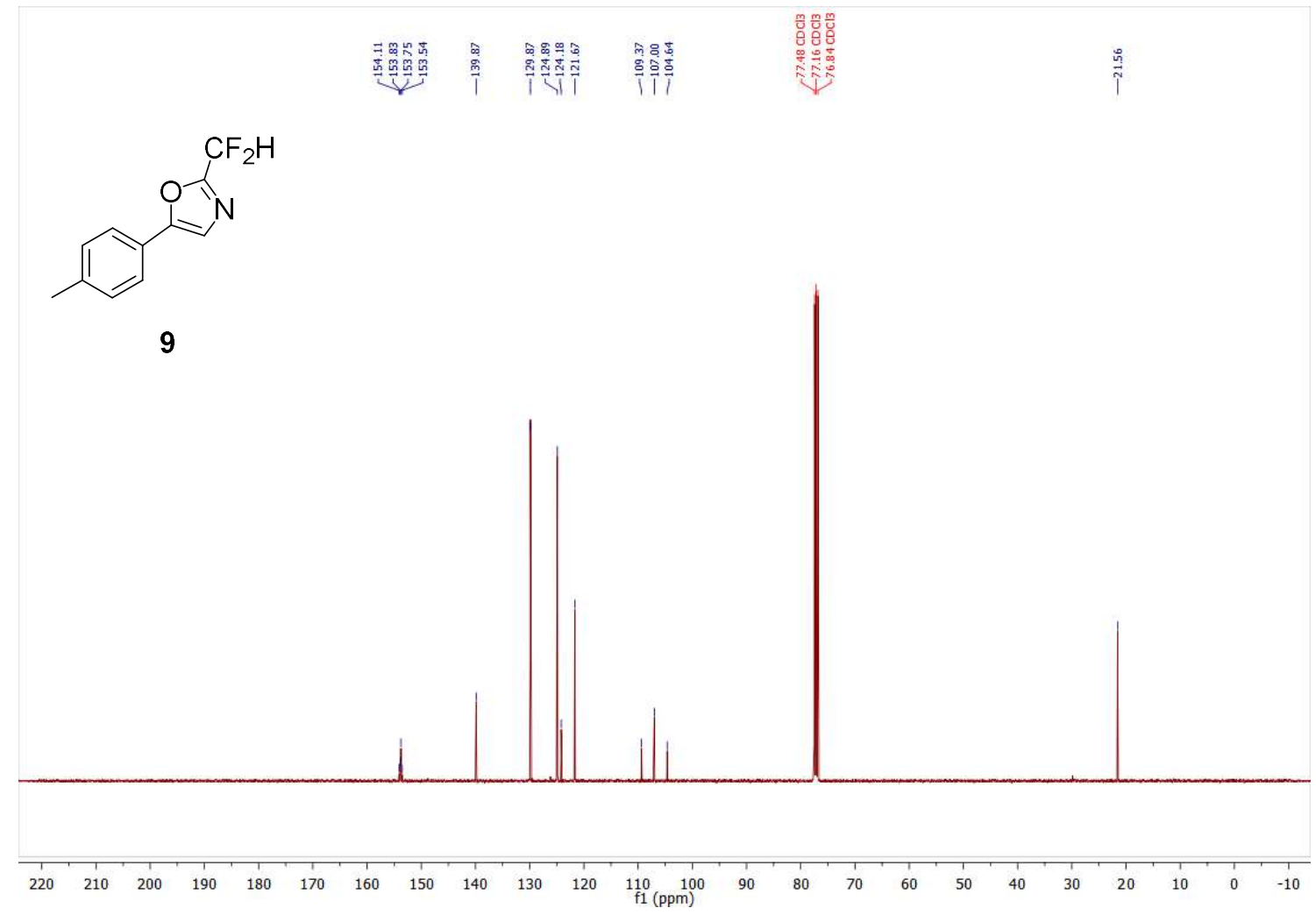

${ }^{19} \mathrm{~F}$ NMR spectrum of $9\left(\mathrm{CDCl}_{3}, 377 \mathrm{MHz}\right)$ 


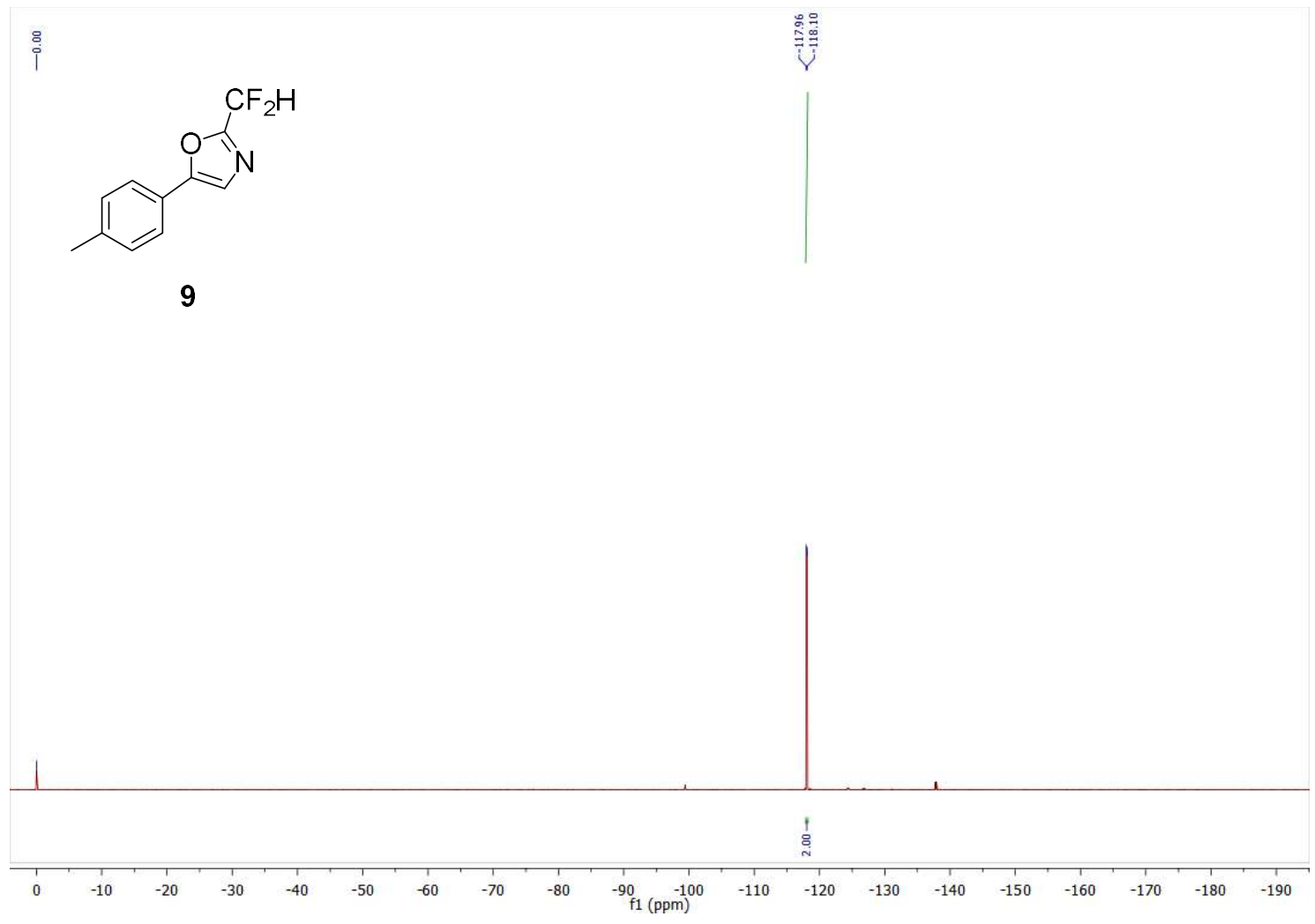

${ }^{1} \mathrm{H}$ NMR spectrum of $\mathbf{1 0}\left(\mathrm{CDCl}_{3}, 400 \mathrm{MHz}\right)$

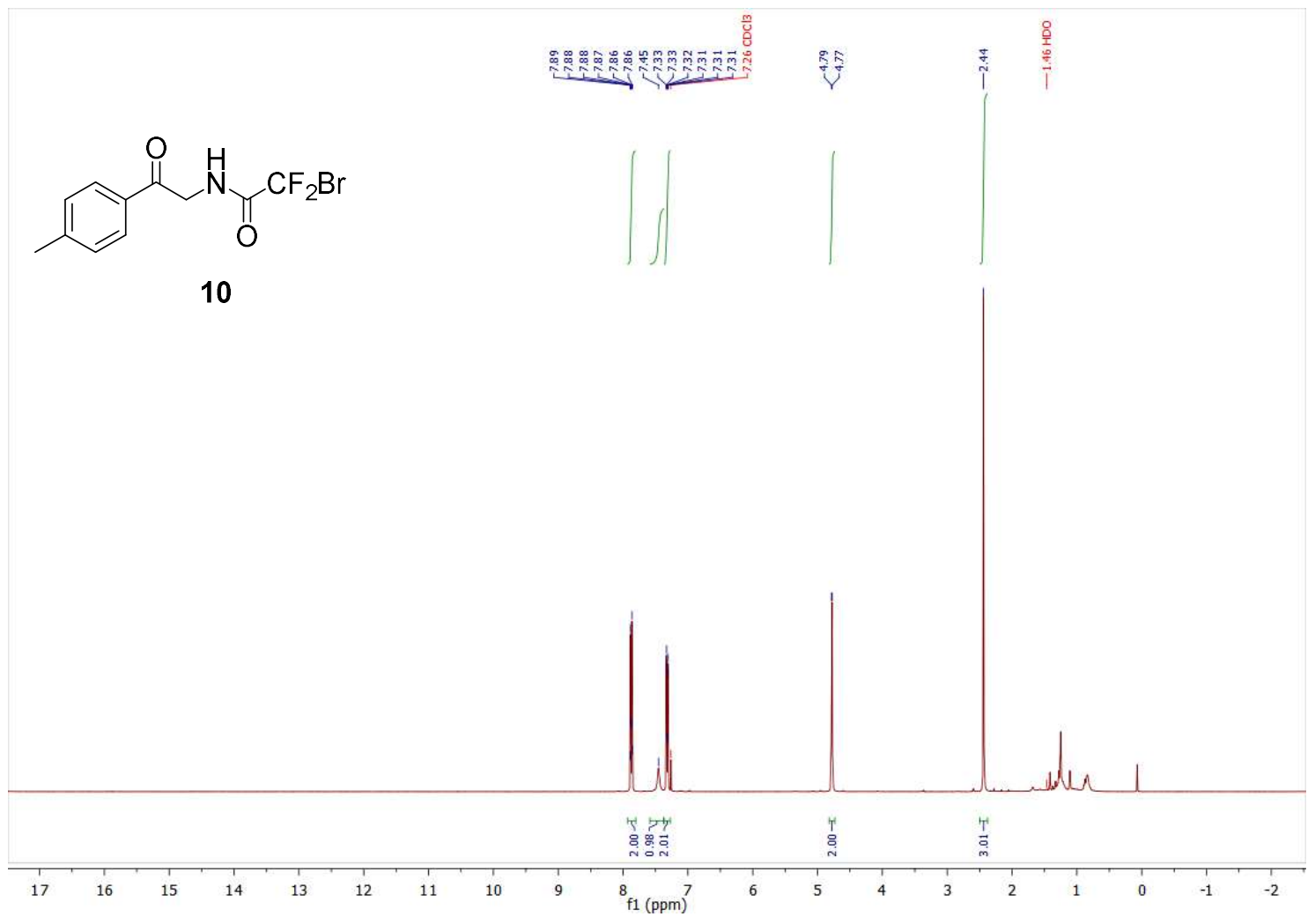

${ }^{13} \mathrm{C}\left\{{ }^{1} \mathrm{H}\right\}$ NMR spectrum of $10\left(\mathrm{CDCl}_{3}, 101 \mathrm{MHz}\right)$ 


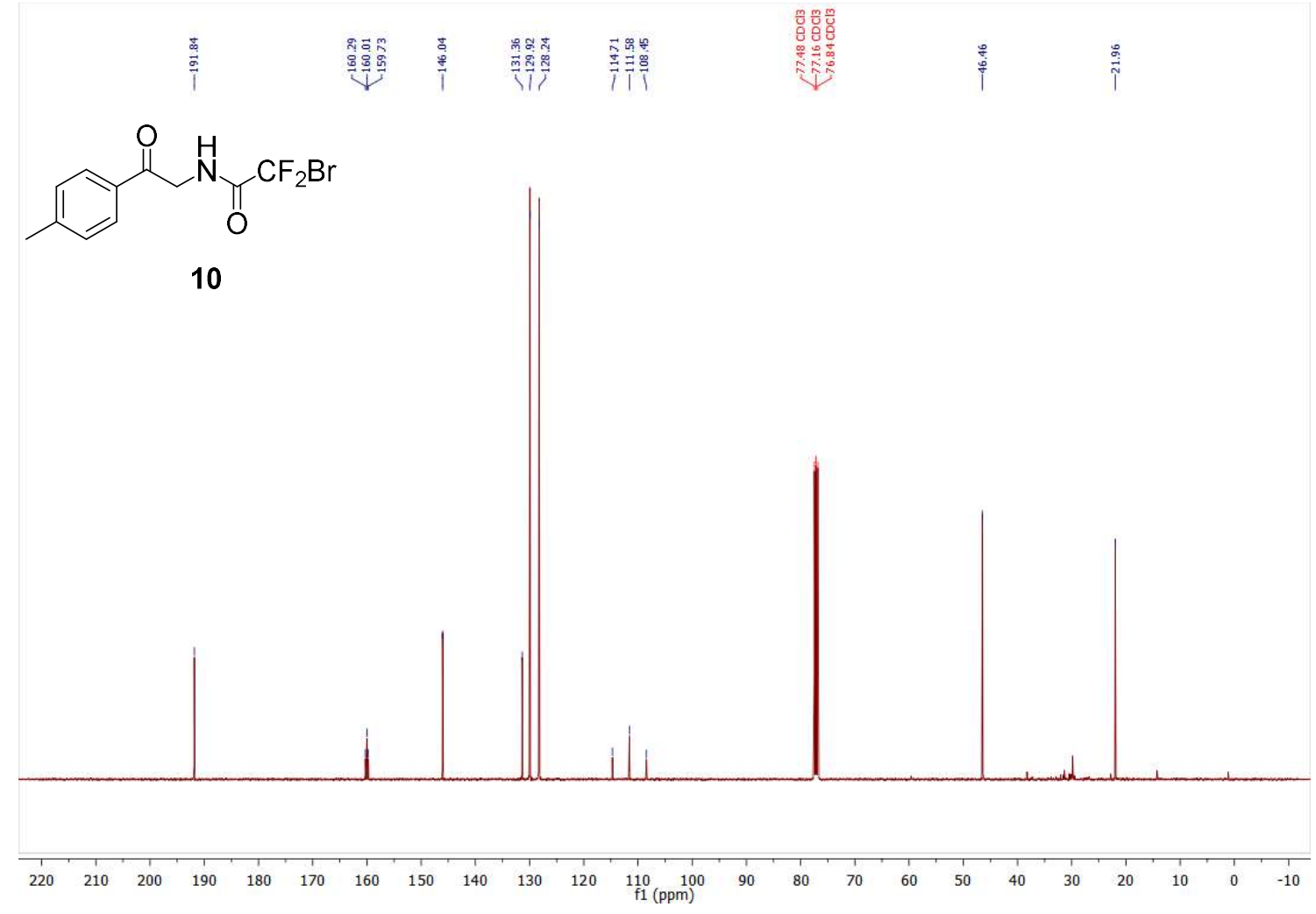

${ }^{19} \mathrm{~F}$ NMR spectrum of $\mathbf{1 0}\left(\mathrm{CDCl}_{3}, 377 \mathrm{MHz}\right)$
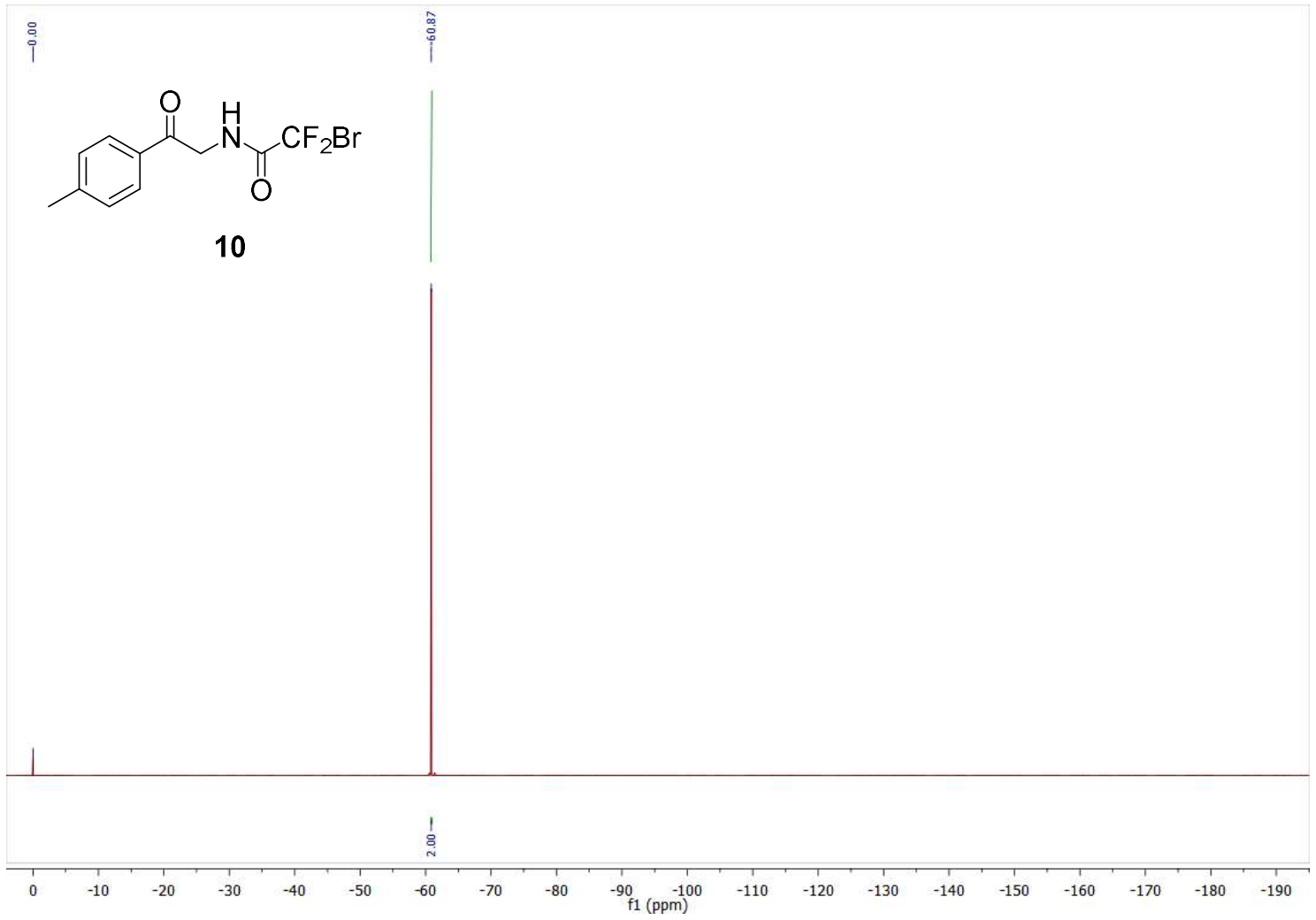

${ }^{1} \mathrm{H}$ NMR spectrum of $11\left(\mathrm{CDCl}_{3}, 400 \mathrm{MHz}\right)$ 
<smiles>O=C(CNC(=O)C(Br)(Br)C(F)(F)Br)c1ccc(F)cc1</smiles>

11

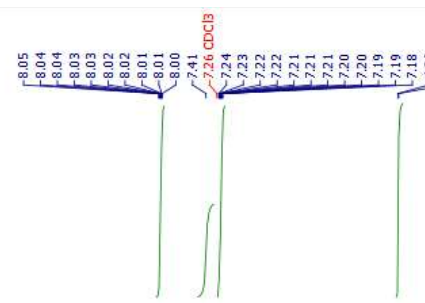

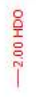

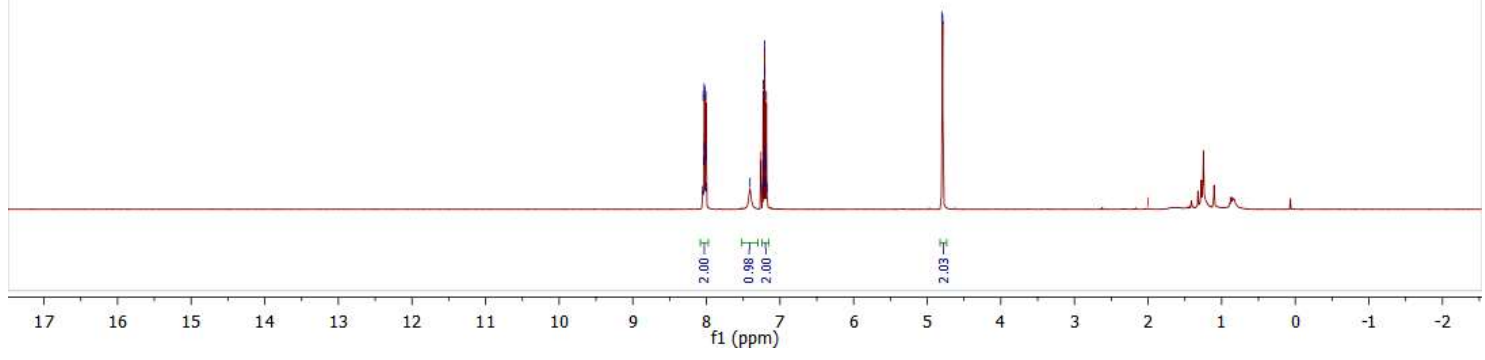

${ }^{13} \mathrm{C}\left\{{ }^{1} \mathrm{H}\right\}$ NMR spectrum of $\mathbf{1 1}\left(\mathrm{CDCl}_{3}, 101 \mathrm{MHz}\right)$
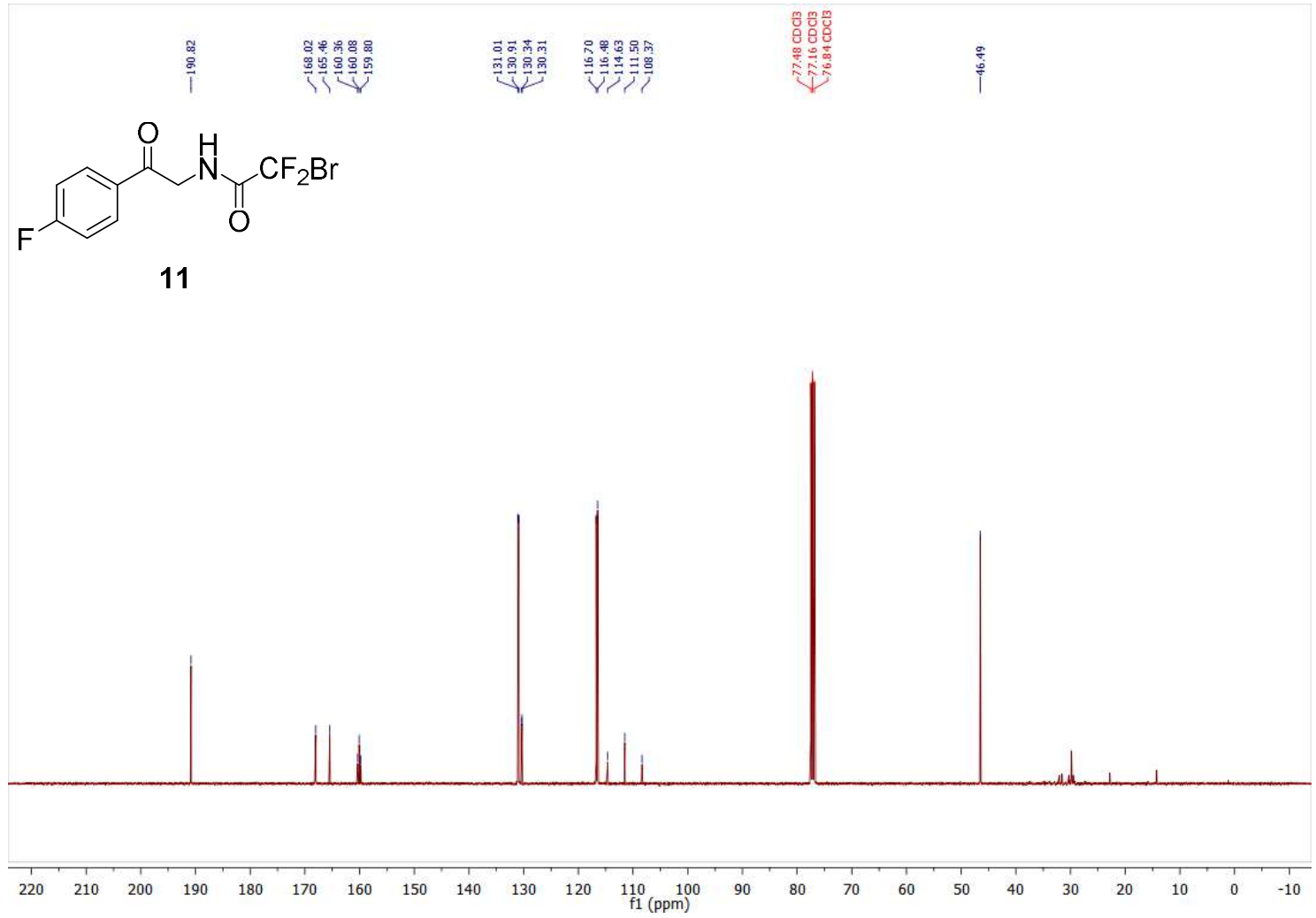

${ }^{19} \mathrm{~F} \mathrm{NMR} \mathrm{spectrum} \mathrm{of} \mathbf{1 1}\left(\mathrm{CDCl}_{3}, 377 \mathrm{MHz}\right)$ 


$$
\text { : }
$$

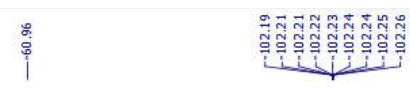<smiles>O=C(CNC(=O)C(F)(Br)C(F)(F)C(F)(F)Br)c1ccc(F)cc1</smiles>

11

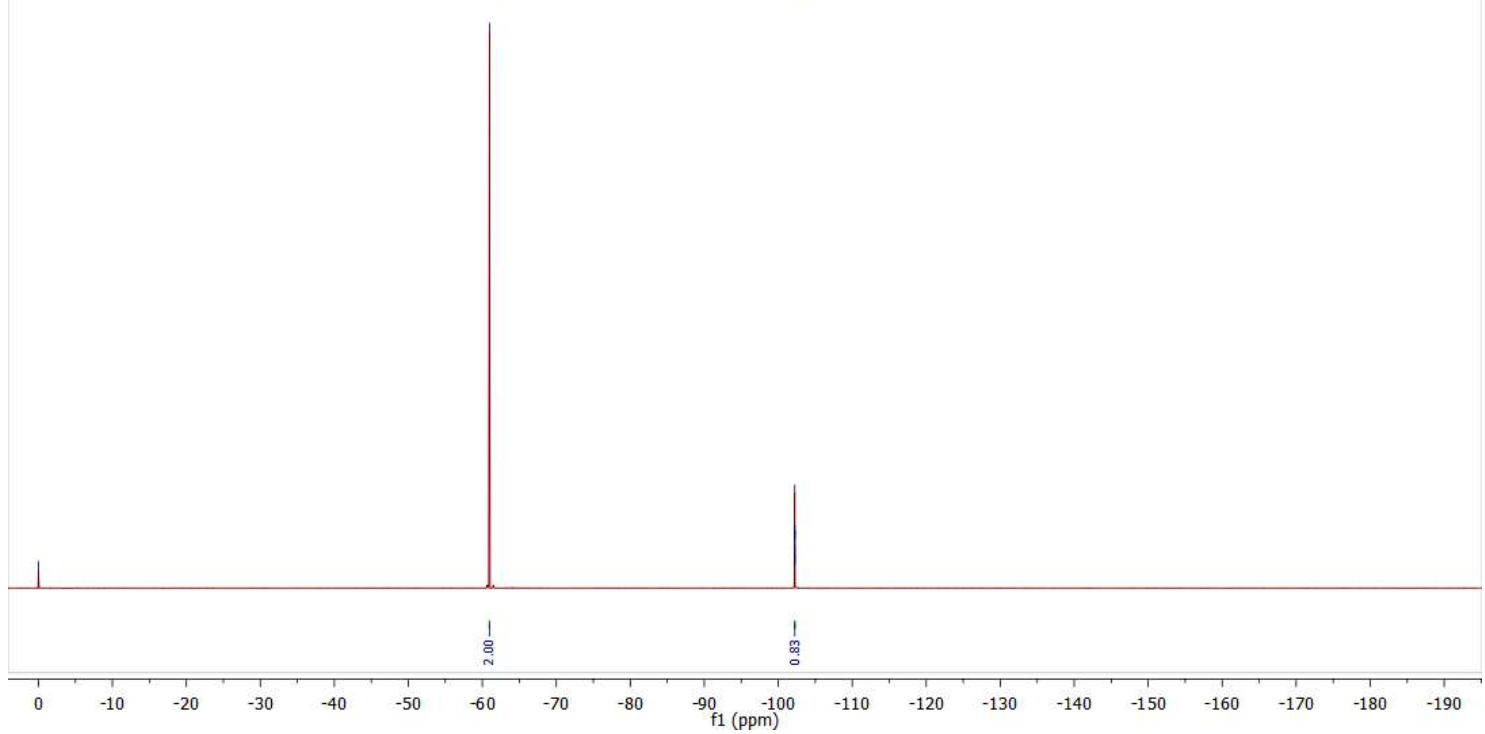

${ }^{1} \mathrm{H}$ NMR spectrum of $13\left(\mathrm{CDCl}_{3}, 400 \mathrm{MHz}\right)$

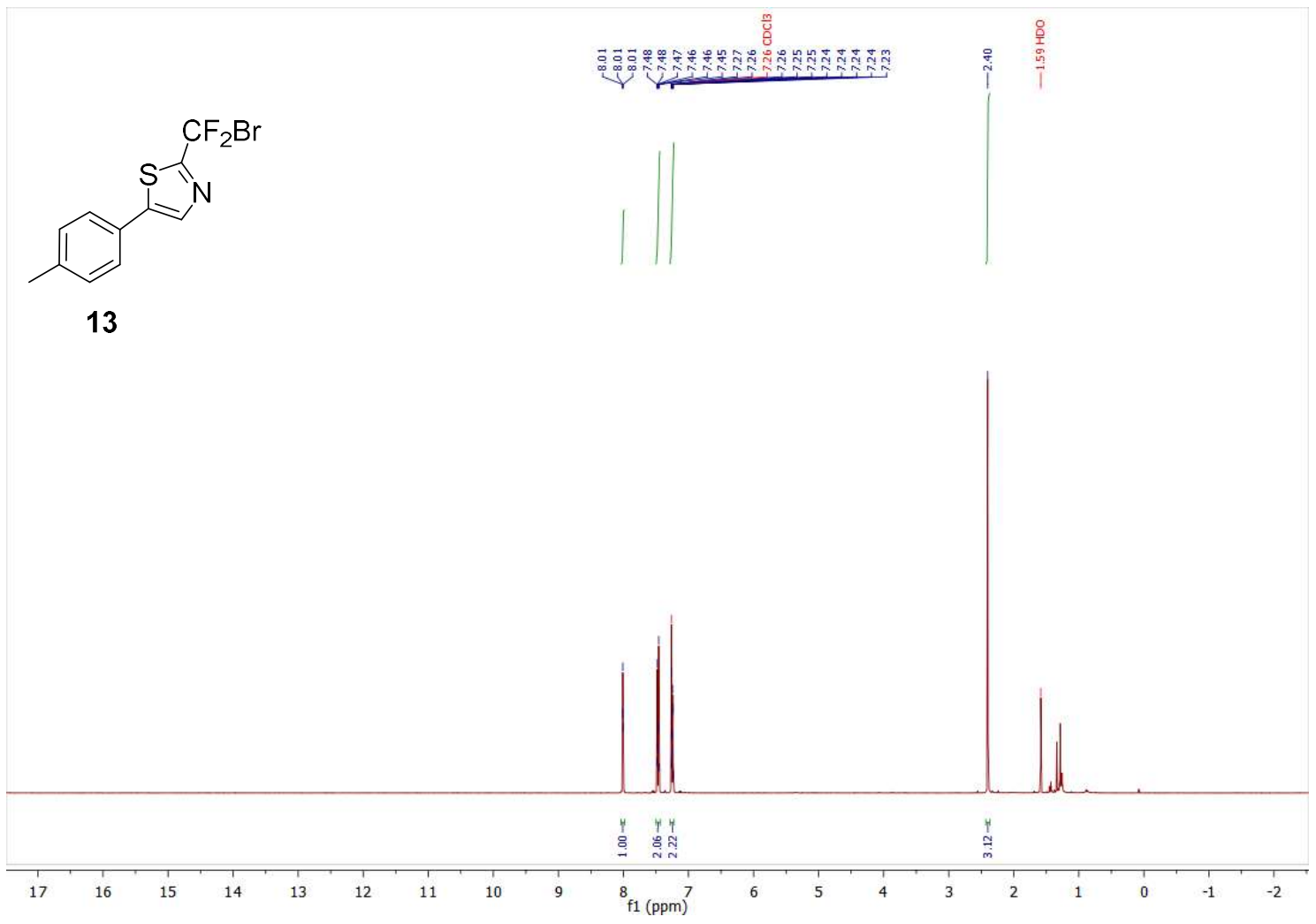

${ }^{13} \mathrm{C}\left\{{ }^{1} \mathrm{H}\right\}$ NMR spectrum of $13\left(\mathrm{CDCl}_{3}, 101 \mathrm{MHz}\right)$ 

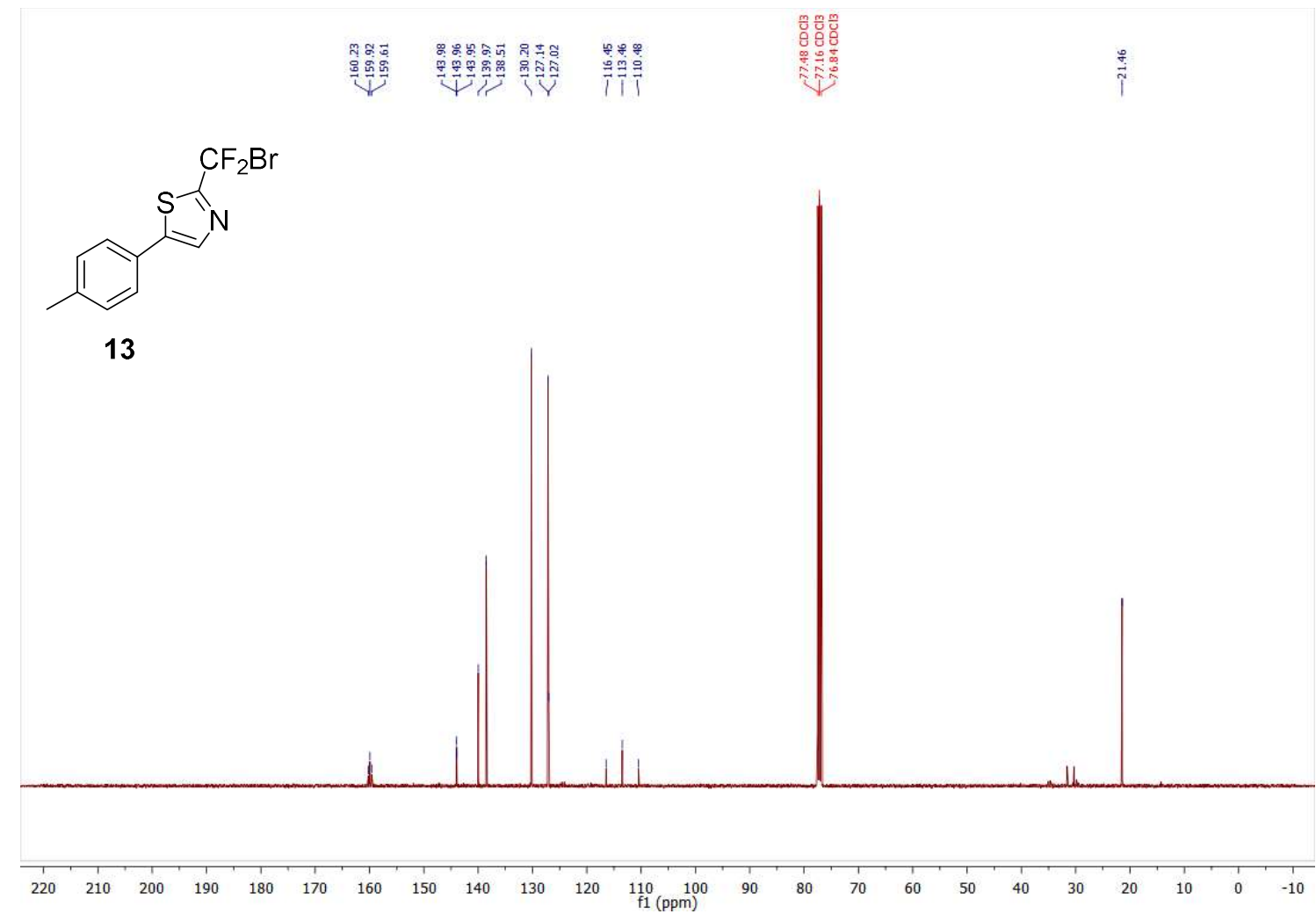

${ }^{19} \mathrm{~F} \mathrm{NMR}$ spectrum of $\mathbf{1 3}\left(\mathrm{CDCl}_{3}, 377 \mathrm{MHz}\right)$

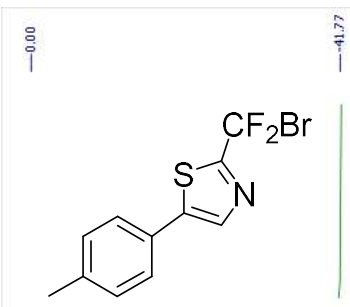

13
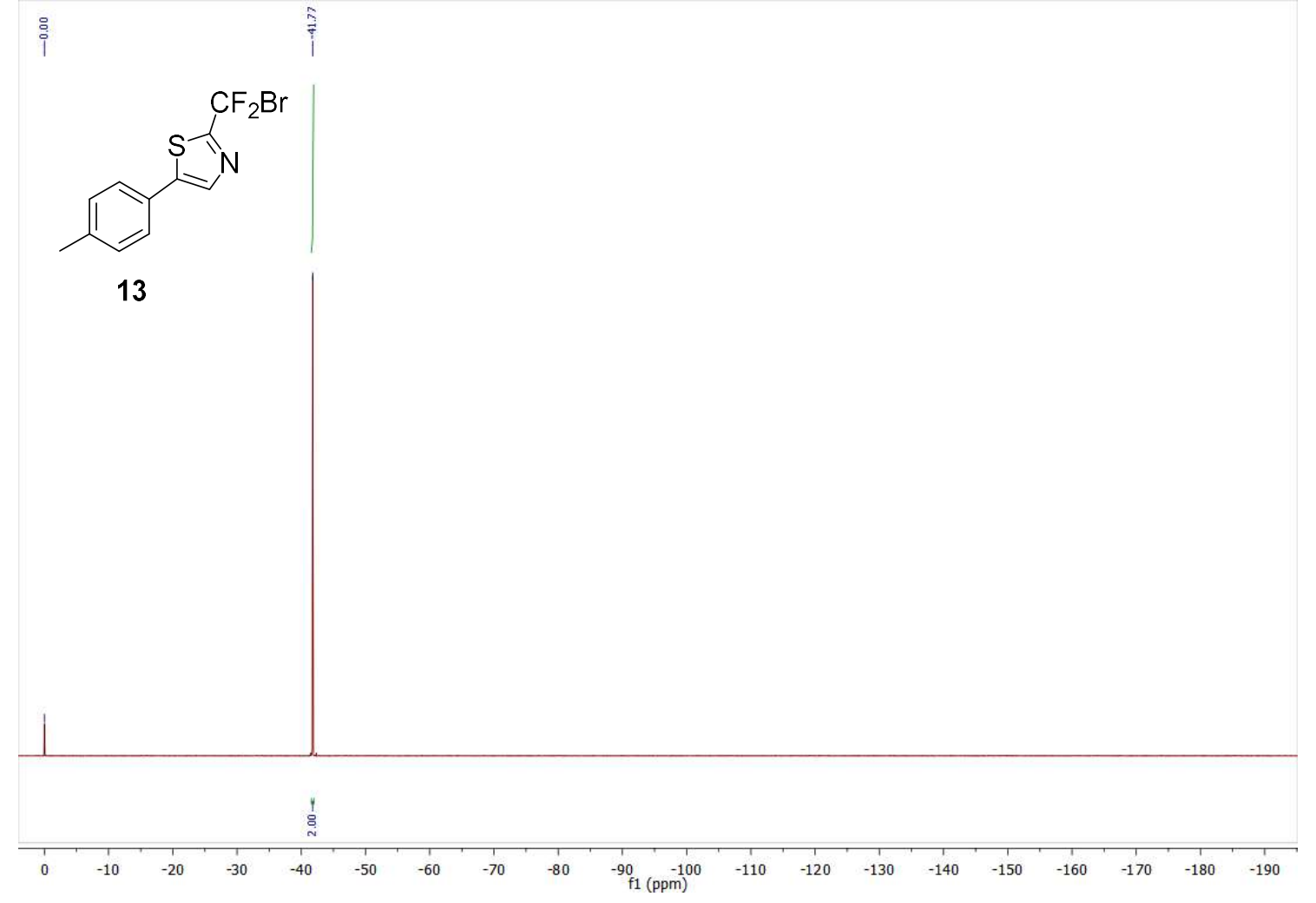

${ }^{1} \mathrm{H}$ NMR spectrum of $14\left(\mathrm{CDCl}_{3}, 400 \mathrm{MHz}\right)$ 


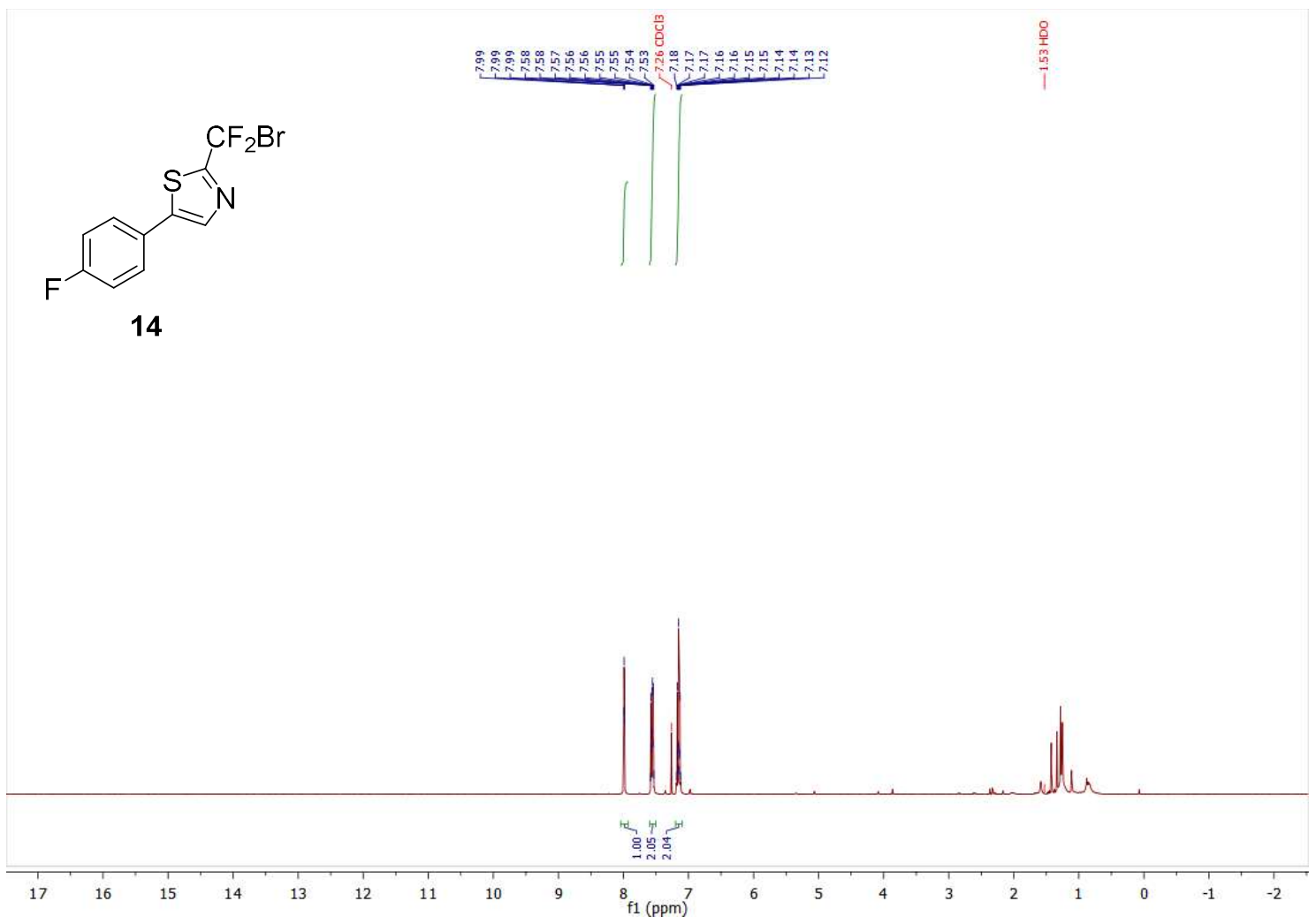

${ }^{13} \mathrm{C}\left\{{ }^{1} \mathrm{H}\right\}$ NMR spectrum of $14\left(\mathrm{CDCl}_{3}, 101 \mathrm{MHz}\right)$

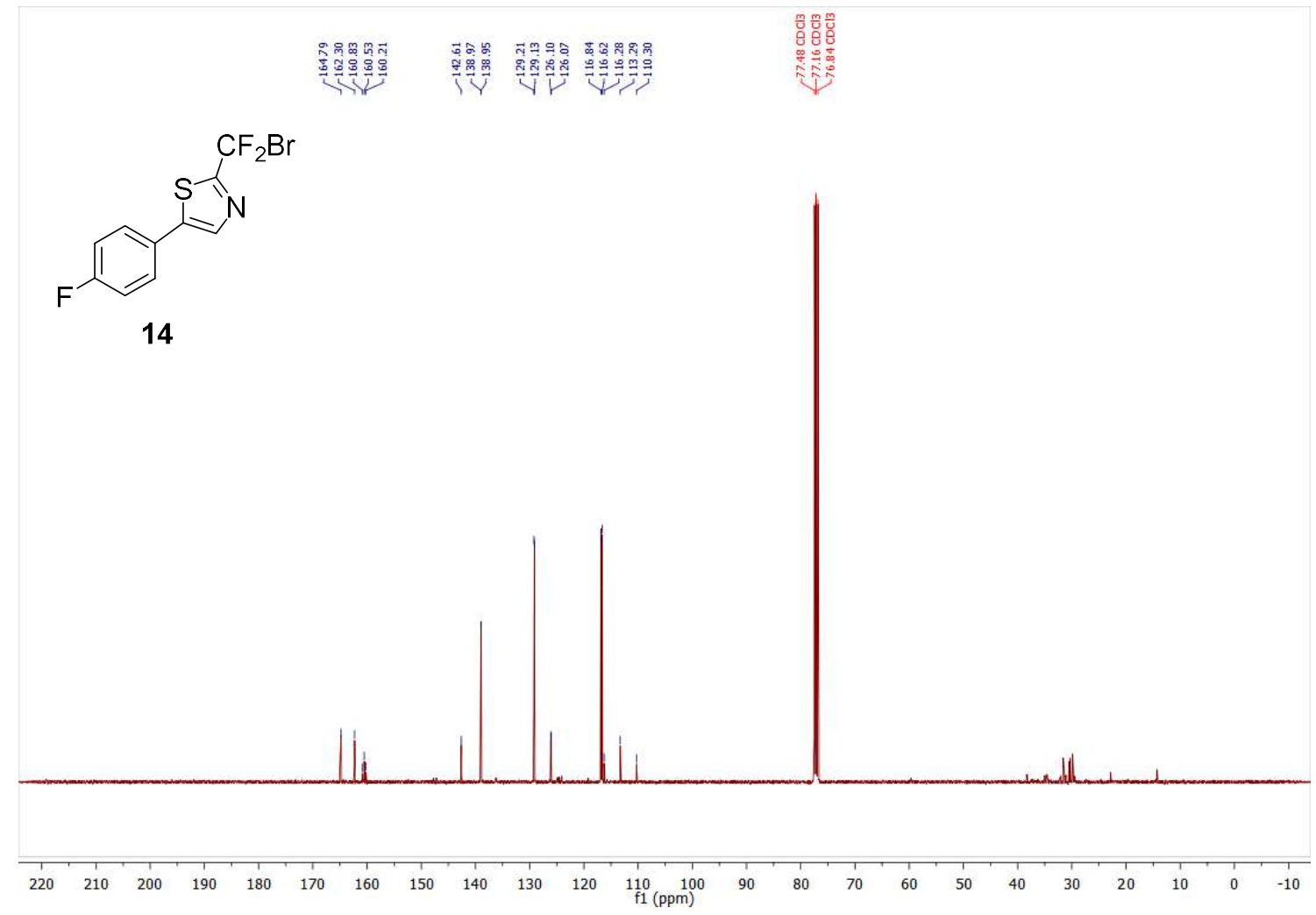

${ }^{19} \mathrm{~F}$ NMR spectrum of $\mathbf{1 4}\left(\mathrm{CDCl}_{3}, 377 \mathrm{MHz}\right)$ 


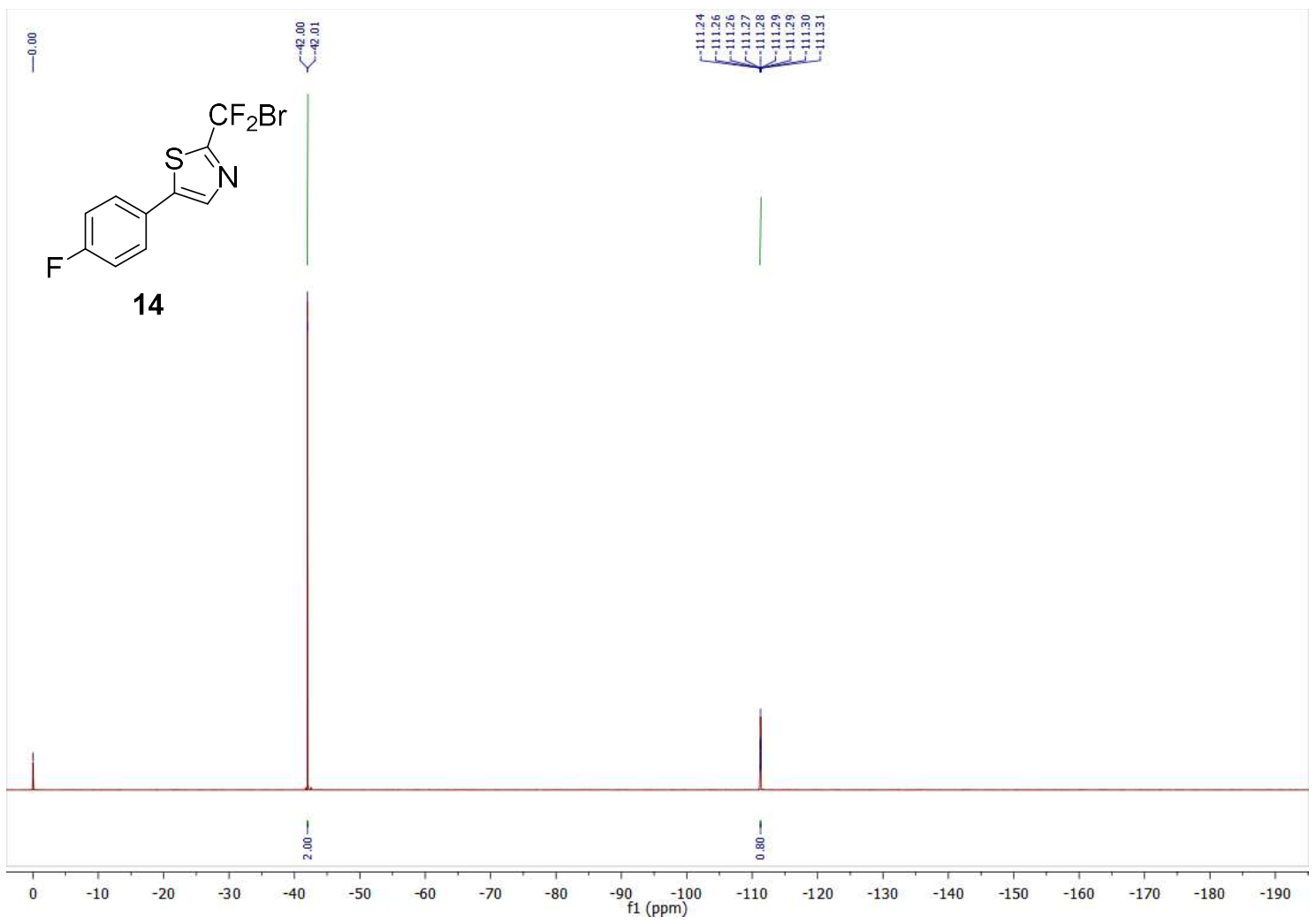

${ }^{1} \mathrm{H}$ NMR spectrum of $15\left(\mathrm{CDCl}_{3}, 400 \mathrm{MHz}\right)$

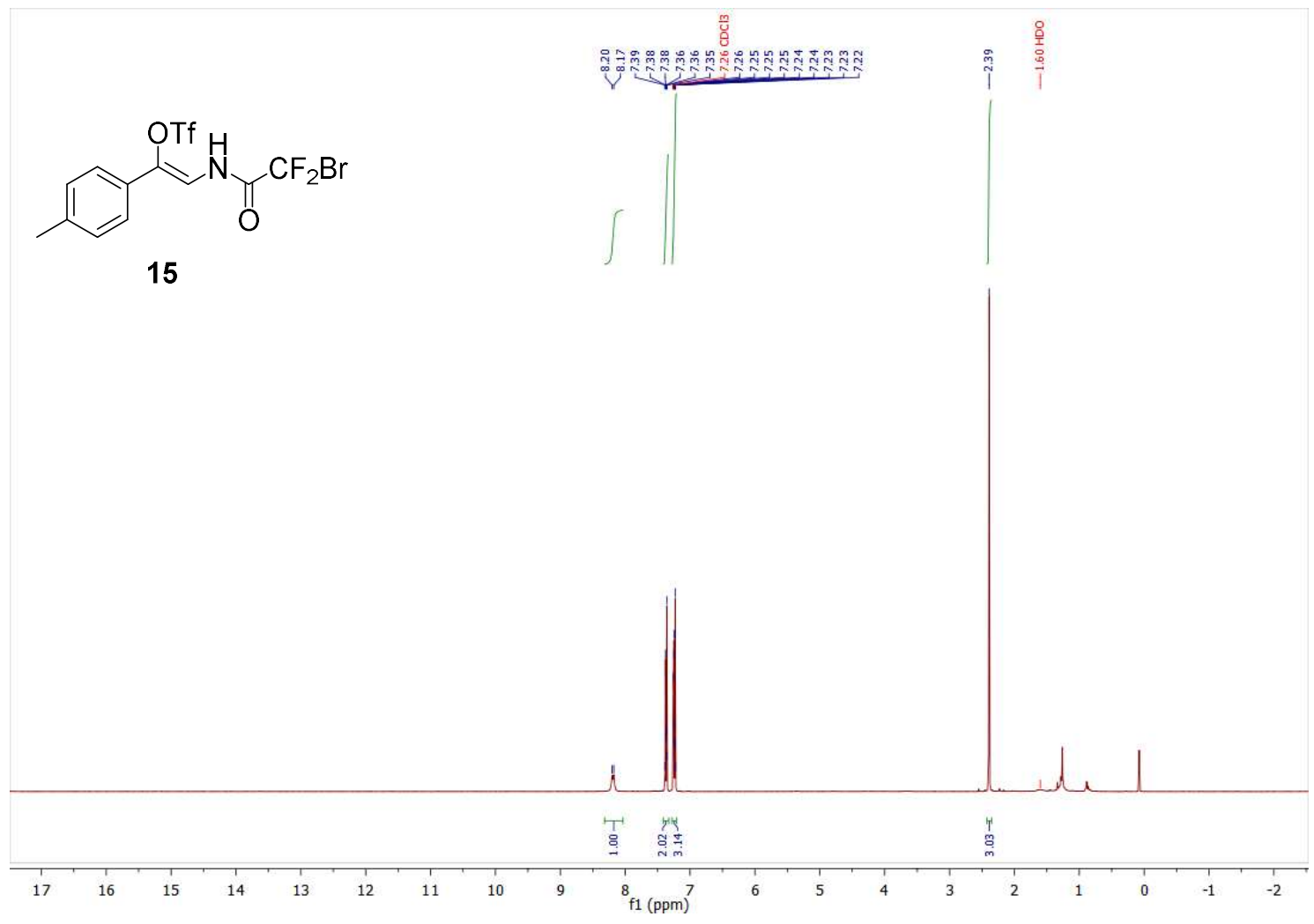

${ }^{13} \mathrm{C}\left\{{ }^{1} \mathrm{H}\right\}$ NMR spectrum of $15\left(\mathrm{CDCl}_{3}, 101 \mathrm{MHz}\right)$ 

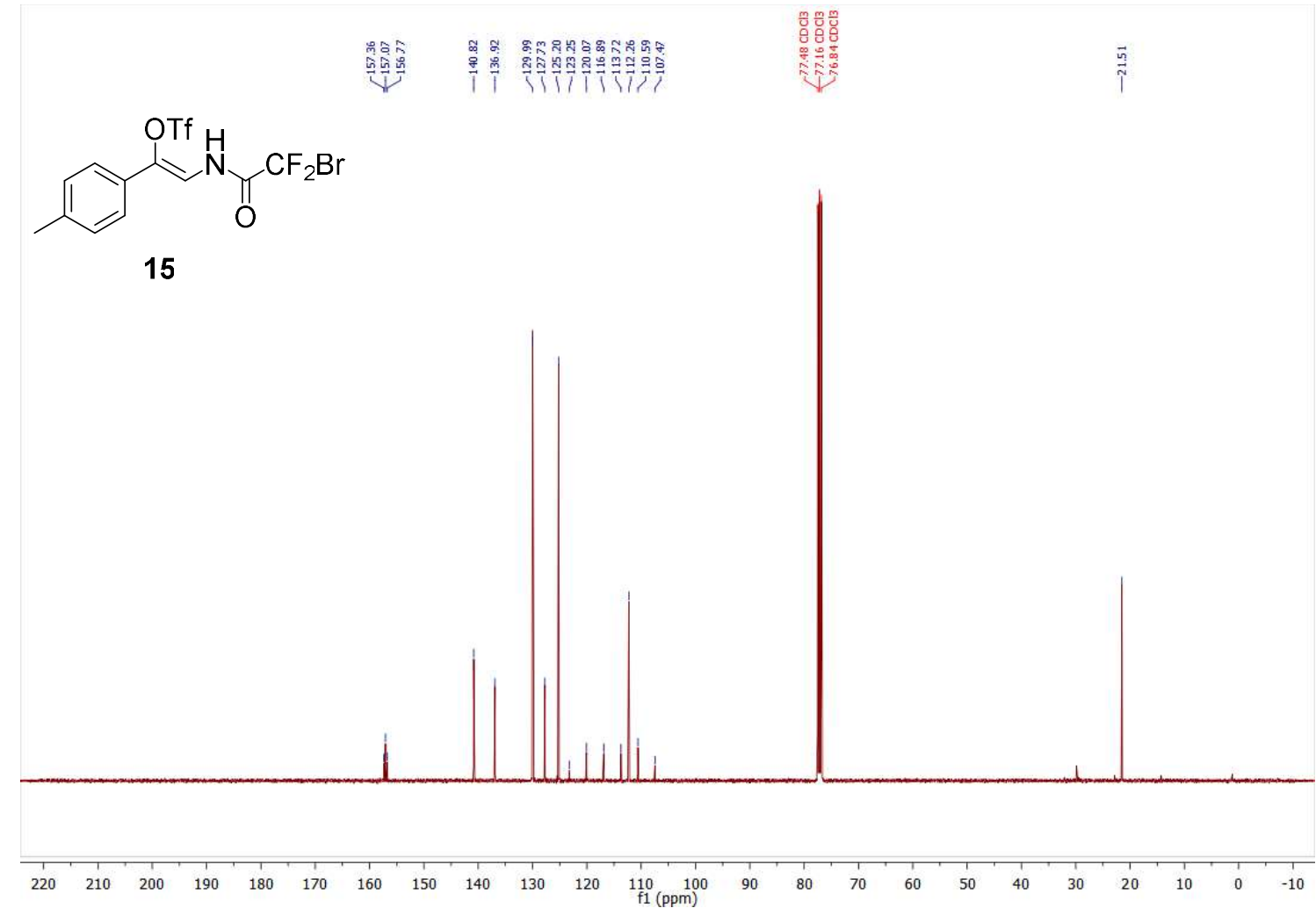

${ }^{19} \mathrm{~F}$ NMR spectrum of $\mathbf{1 5}\left(\mathrm{CDCl}_{3}, 377 \mathrm{MHz}\right)$
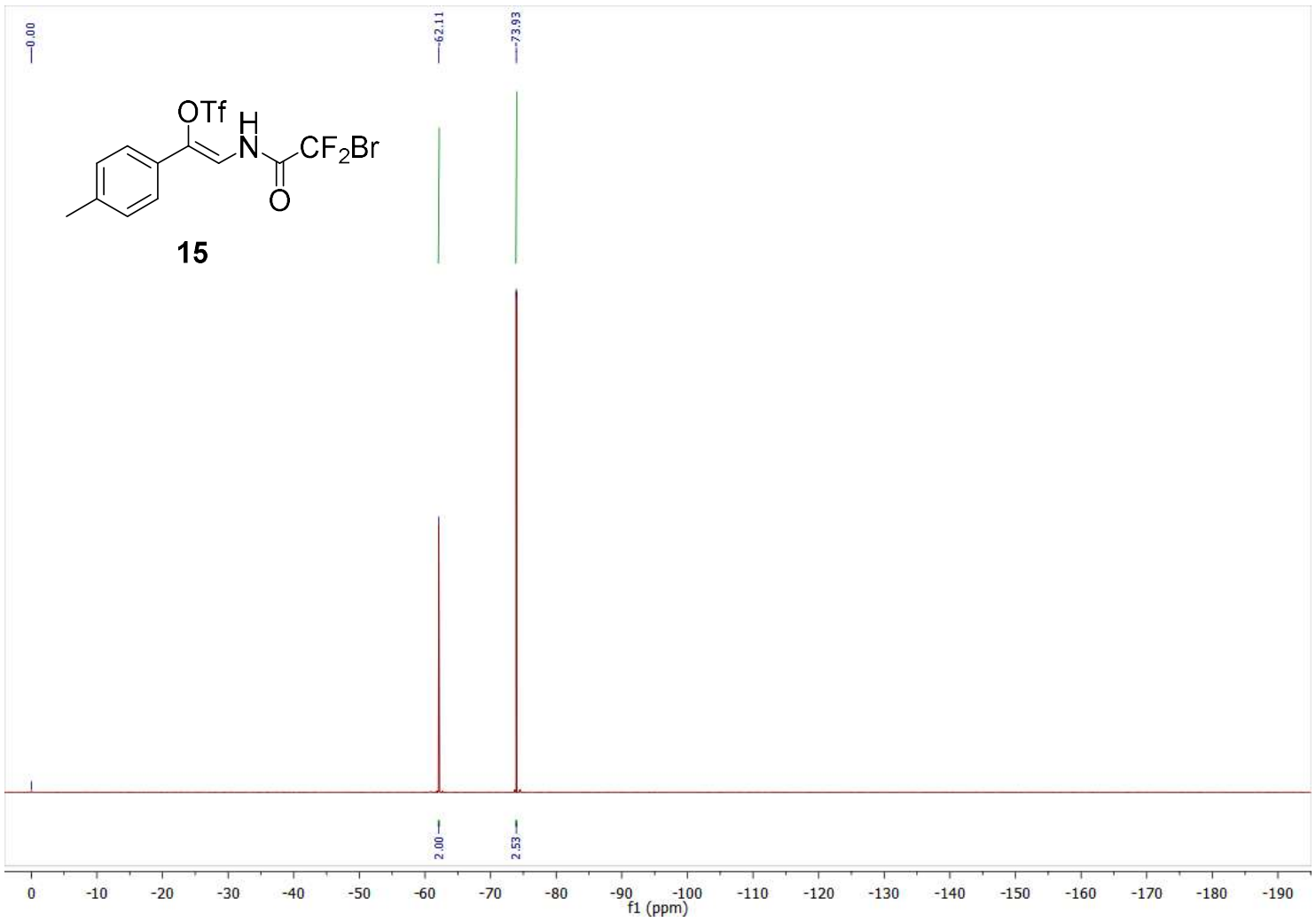

${ }^{1} \mathrm{H}$ NMR spectrum of $16\left(\mathrm{CDCl}_{3}, 400 \mathrm{MHz}\right)$ 


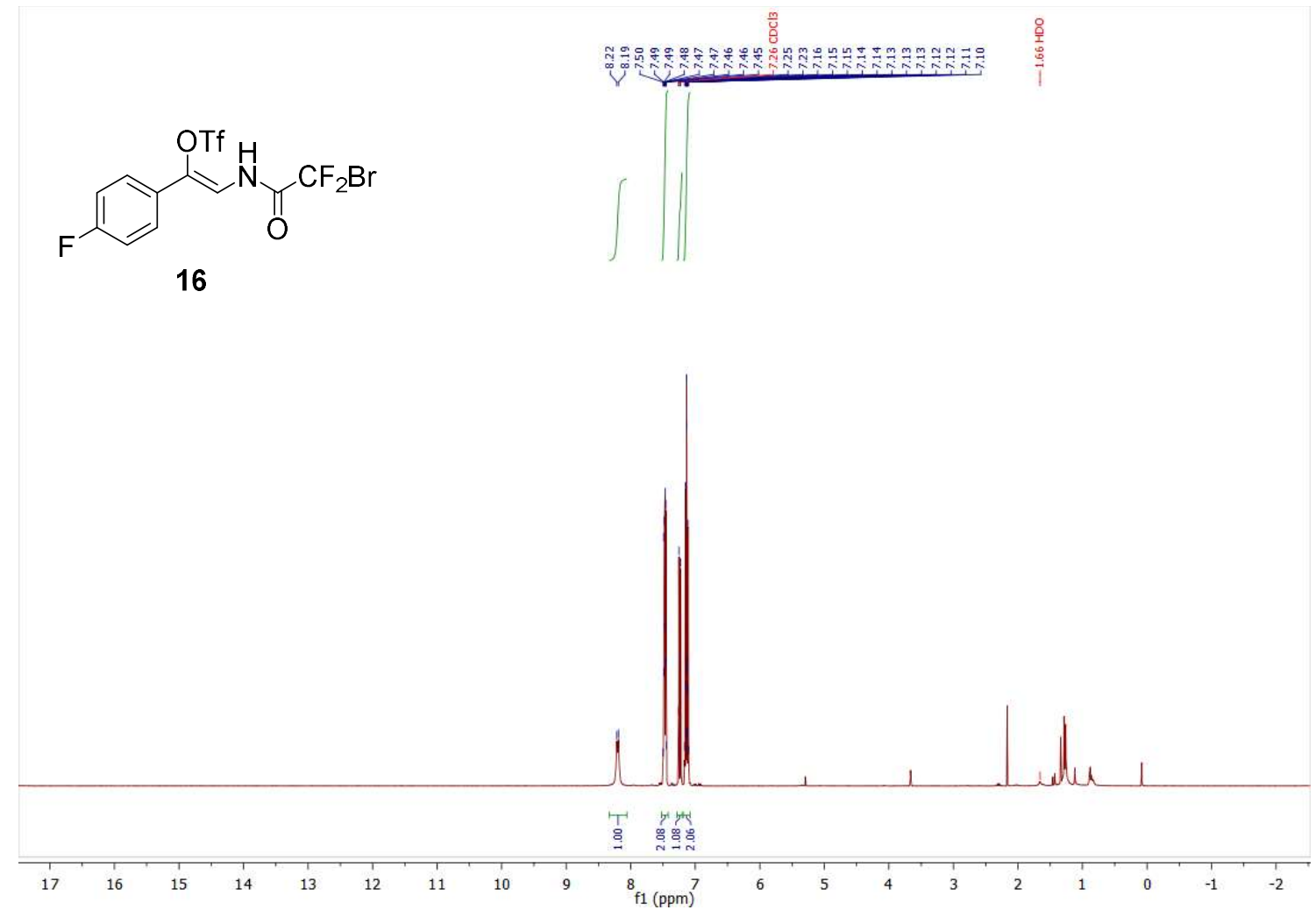

${ }^{13} \mathrm{C}\left\{{ }^{1} \mathrm{H}\right\}$ NMR spectrum of $16\left(\mathrm{CDCl}_{3}, 101 \mathrm{MHz}\right)$
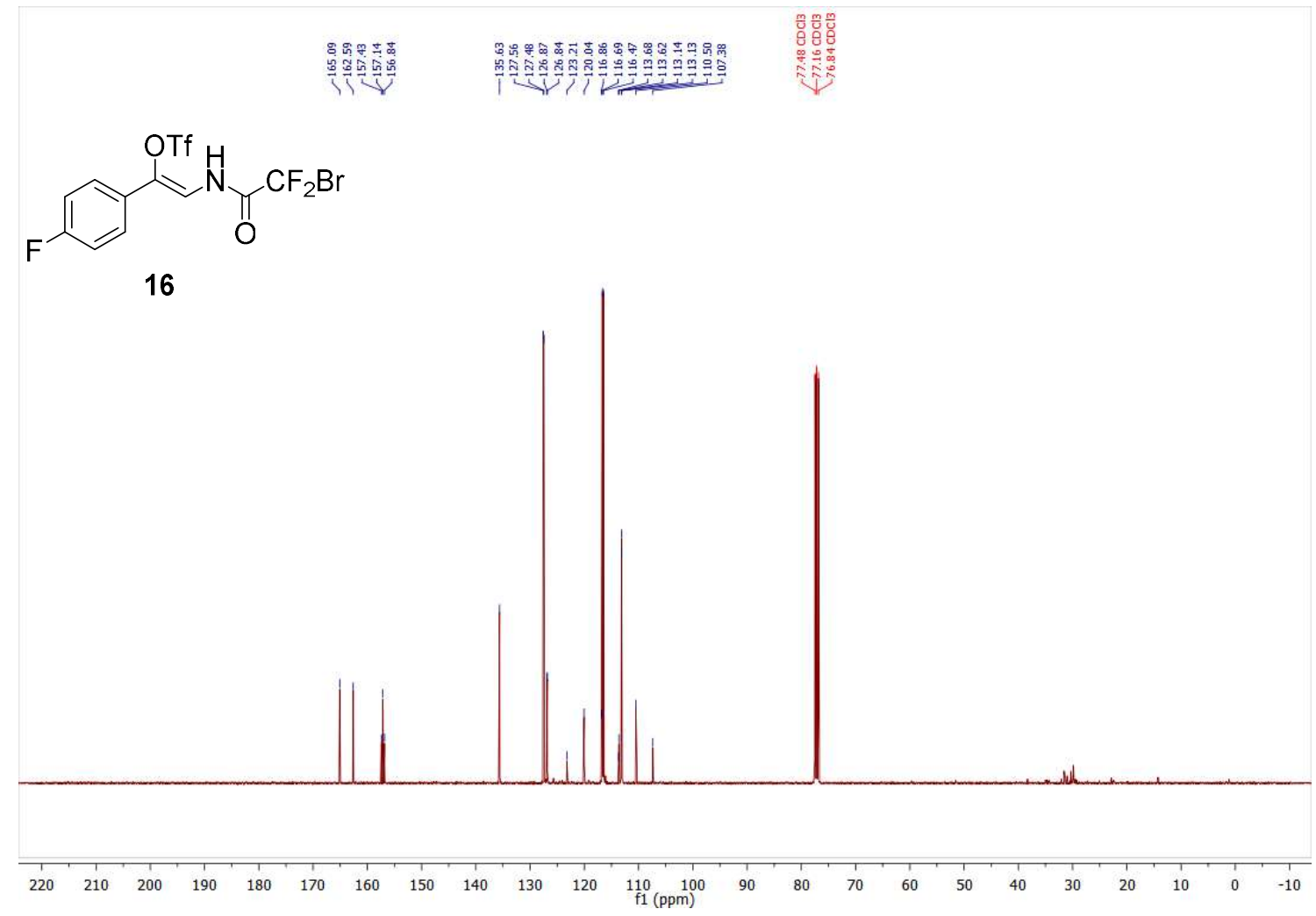

${ }^{19} \mathrm{~F}$ NMR spectrum of $\mathbf{1 6}\left(\mathrm{CDCl}_{3}, 377 \mathrm{MHz}\right)$ 


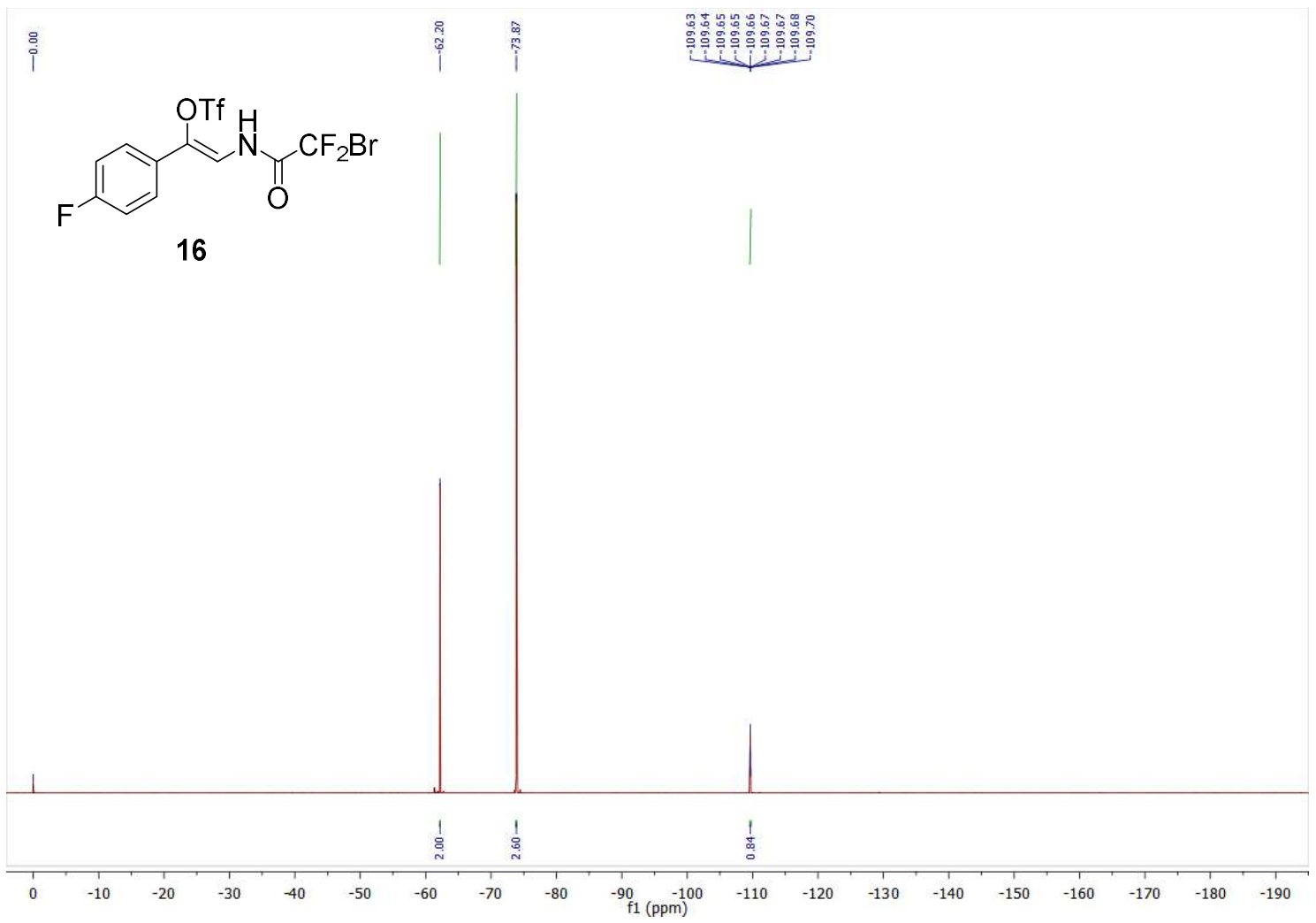

${ }^{1} \mathrm{H}$ NMR spectrum of $17\left(\mathrm{CDCl}_{3}, 400 \mathrm{MHz}\right)$

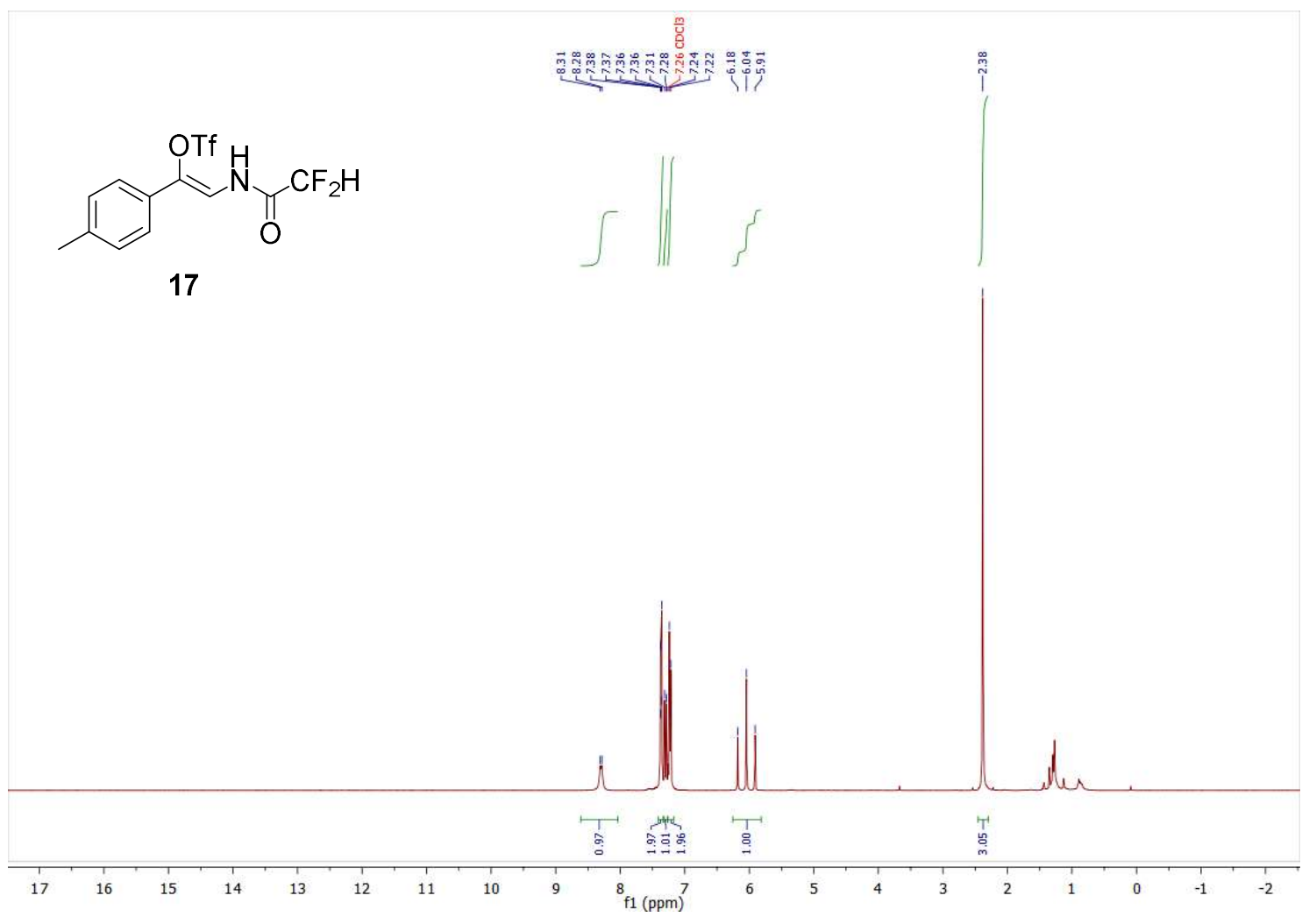

${ }^{13} \mathrm{C}\left\{{ }^{1} \mathrm{H}\right\}$ NMR spectrum of $\mathbf{1 7}\left(\mathrm{CDCl}_{3}, 101 \mathrm{MHz}\right)$ 

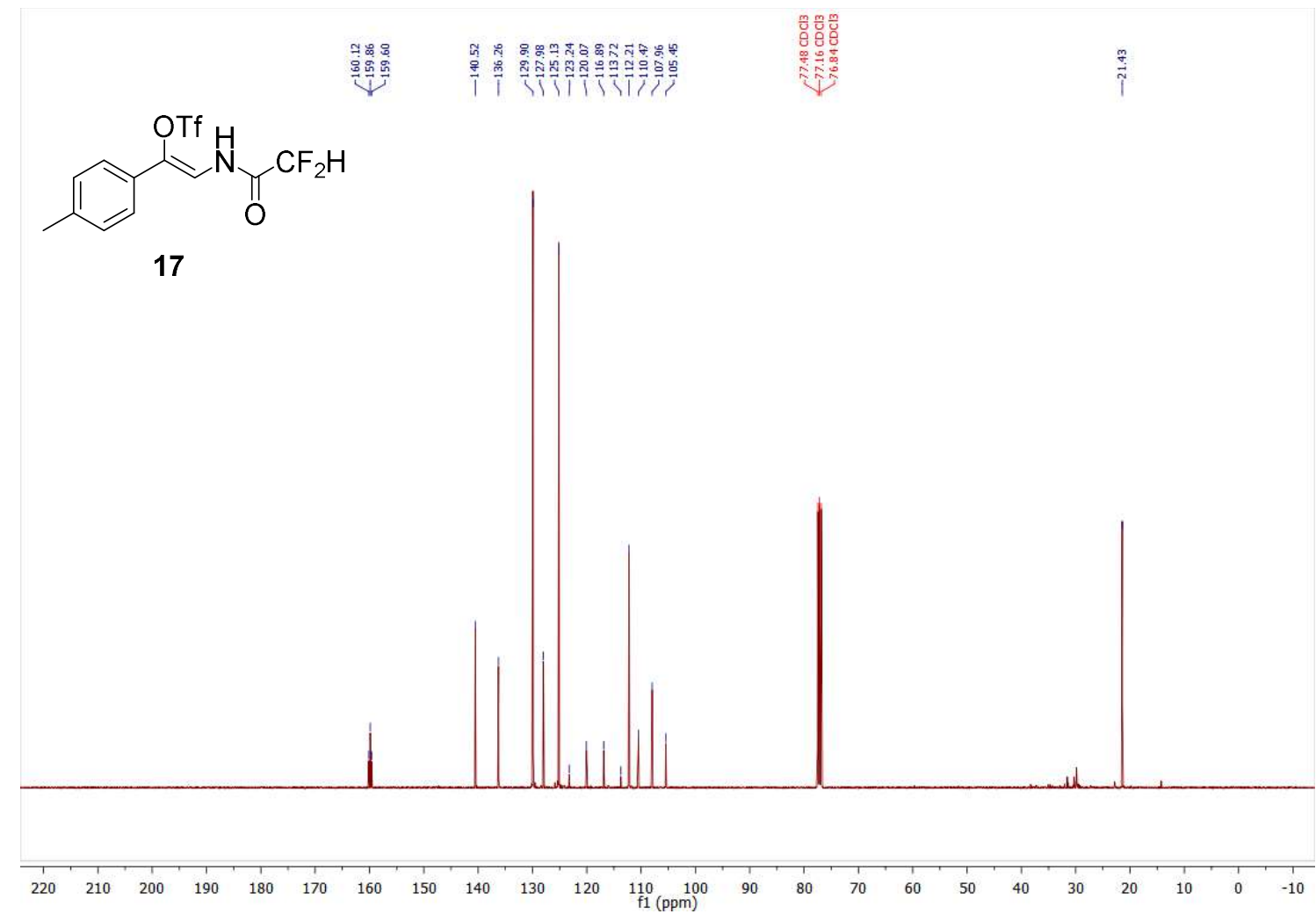

${ }^{19} \mathrm{~F}$ NMR spectrum of $\mathbf{1 7}\left(\mathrm{CDCl}_{3}, 377 \mathrm{MHz}\right)$

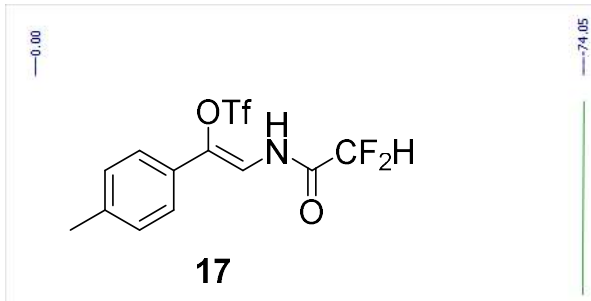

온
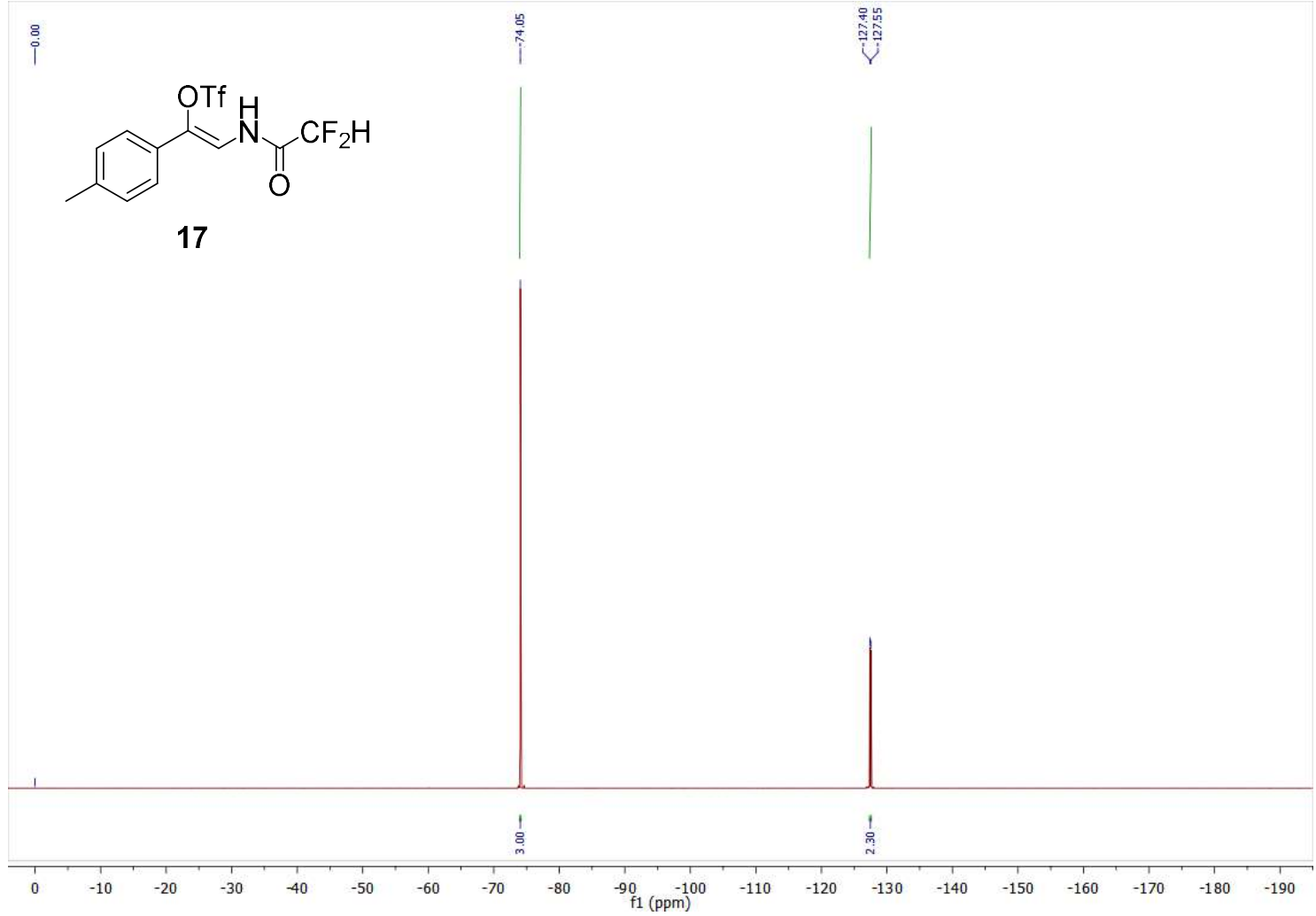